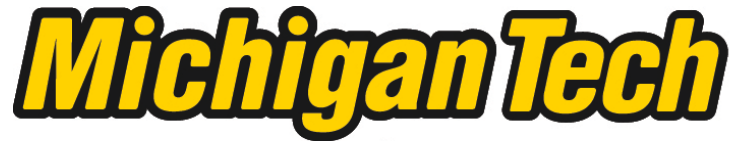 \\ Michigan Technological University Create the Future Digital Commons @ Michigan Tech
}

2015

\section{MULTISCALE EXAMINATION AND MODELING OF ELECTRON TRANSPORT IN NANOSCALE MATERIALS AND DEVICES}

Douglas R. Banyai

Michigan Technological University

Follow this and additional works at: https://digitalcommons.mtu.edu/etds

Part of the Condensed Matter Physics Commons, Nanoscience and Nanotechnology Commons, and the Quantum Physics Commons

Copyright 2015 Douglas R. Banyai

\section{Recommended Citation}

Banyai, Douglas R., "MULTISCALE EXAMINATION AND MODELING OF ELECTRON TRANSPORT IN NANOSCALE MATERIALS AND DEVICES", Dissertation, Michigan Technological University, 2015.

https://doi.org/10.37099/mtu.dc.etds/947

Follow this and additional works at: https://digitalcommons.mtu.edu/etds

Part of the Condensed Matter Physics Commons, Nanoscience and Nanotechnology Commons, and the Quantum Physics Commons 


\title{
MULTISCALE EXAMINATION AND MODELING OF ELECTRON TRANSPORT IN NANOSCALE MATERIALS AND DEVICES
}

\author{
By
}

Douglas R. Banyai

\begin{abstract}
A DISSERTATION
Submitted in partial fulfillment of the requirements for the degree of DOCTOR OF PHILOSOPHY

In Physics
\end{abstract}

MICHIGAN TECHNOLOGICAL UNIVERSITY

2015

(C) 2015 Douglas R. Banyai 

This dissertation has been approved in partial fulfillment of the requirements for the Degree of DOCTOR OF PHILOSOPHY in Physics.

Department of Physics
Dissertation Advisor: Dr. John A. Jaszczak
Committee Member: Dr. Ravindra Pandey
Committee Member: Dr. Paul L. Bergstrom
Committee Member: Dr. Loredana Valenzano
Department Chair: Dr. Ravindra Pandey 

To my parents. 



\section{Contents}

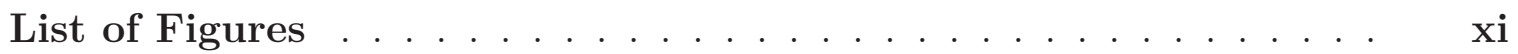

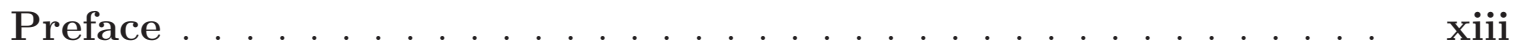

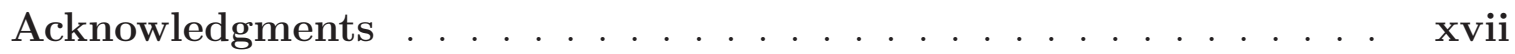

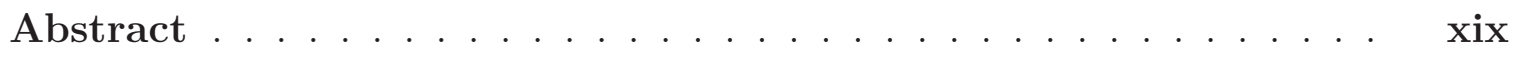

1 Introduction . . . . . . . . . . . . . . . . . . 1

1.1 Modern Electronics . . . . . . . . . . . . . . . . . . . . 2

1.1.1 The Perpetually Impending End of Moore's Law . . . . . . . 2

1.1.2 New Transistor Designs Use Tunneling to Their Advantage . 3

1.1.3 Randomly Created Devices with Randomized Properties . . 4

1.1.4 Simulation of SETs . . . . . . . . . . . . . . . . 4

1.2 Scanning Tunneling Microscopy and Spectroscopy . . . . . . . . 5

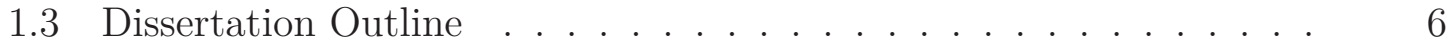

2 Simulation of Disordered Coulomb Blockade Devices . . . . . . 9

2.1 Abstract . . . . . . . . . . . . . . . . . . . 9

2.2 Introduction . . . . . . . . . . . . . . . . . 10

2.3 Theory and Simulation . . . . . . . . . . . . . . . . . 12

2.4 Results . . . . . . . . . . . . . . . . . . . . 17

2.4 .1 One-dimensional devices $\ldots \ldots \ldots \ldots$

2.4 .2 Two-dimensional devices . . . . . . . . . . . . . . . . 19 
2.5 Discussion . . . . . . . . . . . . . . . . . . . . . . . . . . 21

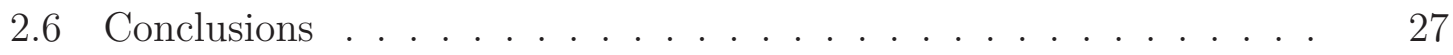

2.7 Acknowledgments . . . . . . . . . . . . . . . . . 27

3 Calculation of Capacitance in Dense Disordered Systems _... 29

3.1 Introduction and Motivation . . . . . . . . . . . . . . . . 29

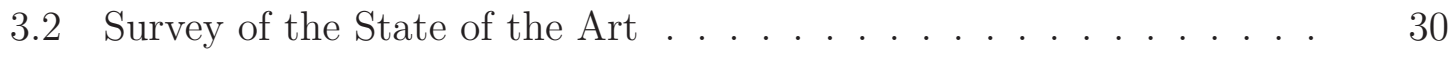

3.3 Computational Methodology . . . . . . . . . . . . . . . . . 32

3.3 .1 Capacitance Matrix . . . . . . . . . . . . . . . . . . 32

3.3.2 Finite Element Calculation . . . . . . . . . . . . . . . 34

3.3 .2 .1 Geometry . . . . . . . . . . . . . 34

$3.3 .2 .2 \quad$ Mesh . . . . . . . . . . . . 36

3.3.2.3 Finite Element Method Calculation . . . . . . . . 37

3.4 Test Systems . . . . . . . . . . . . . . . . . . . . . . . . 38

3.4 .1 Sphere Within a Sphere . . . . . . . . . . . 38

3.4 .2 Single Impinging Sphere . . . . . . . . . . . . . . . . 39

3.4.3 Multiple Surrounding Spheres . . . . . . . . . . . . . 42

3.4.4 Potential Energy Barriers _. . . . . . . . . . . . . 44

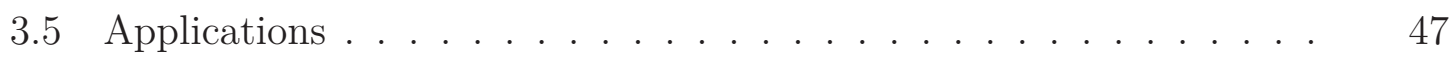

3.5.1 Linear Chain . . . . . . . . . . . . . . . . . 48

3.5 .2 Bent Chain . . . . . . . . . . . . . . . . 52

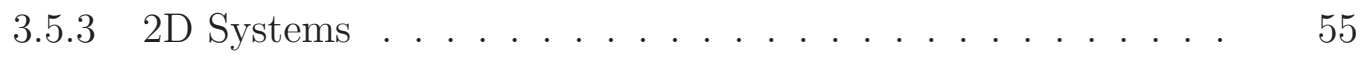

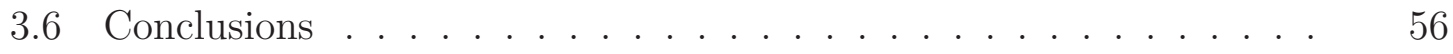

4 Tunneling Resistance Between Clusters _. . . . . . . . . . 59

4.1 Introduction . . . . . . . . . . . . . . . . . 59

4.2 Effect of Resistances on Coulomb Blockade Devices . . . . . . . 60

4.3 Tunneling Through Thin Films . . . . . . . . . . . . . . 62

4.3.1 Tunnel Barriers from Finite Element Method Calculations . 63 
4.4 Tunneling Between Nanoscale Particles . . . . . . . . . . . . 64

4.4.1 Density Functional Theory . . . . . . . . . . . . . . . 64

4.4 Geometry ....................... 66

4.4 .3 Results..................... . . 67

4.4.4 The Hexagon Cluster Mystery . . . . . . . . . . . . . . . 70

4.5 Conclusion ............................ 73

\section{Simulation of Scanning Tunneling Microscopy and Spectroscopy 75}

5.1 Introduction . . . . . . . . . . . . . . . . 75

5.1.1 Methods for computational STM . . . . . . . . . . 76

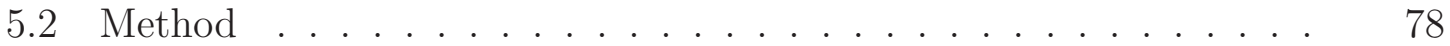

5.2.1 The STM Theory of Tersoff and Hamann . . . . . . . . . . . 78

5.2.2 The STS approximation of Lang . . . . . . . . . . . . . . 79

5.2.3 Superposition of atomic charge-density . . . . . . . . . . 80

5.2.4 SLSTM ...................... 80

5.3 Application to $\mathrm{Si}(111)-(7 \times 7) \ldots \ldots \ldots \ldots$. . . . . . . . . 81

5.3.1 Model ........................ . . 81

5.3.2 Results...................... . . . 83

5.3.3 Discussion . . . . . . . . . . . . . . . . 87

5.4 Application to $(\mathrm{PbS})_{32} \ldots \ldots \ldots \ldots$

5.4.1 Introduction . . . . . . . . . . . . . . . . 90

5.4.2 Model .......................... . . . . 92

5.4.3 Results and Discussion . . . . . . . . . . . . . . . 93

5.4.4 Conclusion . . . . . . . . . . . . . . . . . . . . . . . . 97

5.5 Application to Si-doped BN Monolayer . . . . . . . . . . . . . . 98

5.5.1 Introduction . . . . . . . . . . . . . . . . . . 98

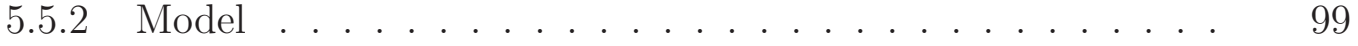

5.5.3 Results and Discussion . . . . . . . . . . . . . . . 102

5.5.4 Conclusion .................... 105 
5.6 Application to $\mathrm{MoS}_{2} \ldots \ldots \ldots \ldots \ldots$

5.6 .1 Introduction . . . . . . . . . . . . . . 106

5.6 .2 Model . . . . . . . . . . . . . . . . . . . . . . . . . 106

5.6.3 Results and Discussion . . . . . . . . . . . . . . 108

5.6 .4 Conclusion . . . . . . . . . . . . . . . . . . . . . . . . 109

$6 \quad$ Future Work and Open Questions . . . . . . . . . . . . . . 111

6.1 Overview . . . . . . . . . . . . . . . . . . . . . . . . . 111

6.2 Detailed Behavior of Individual Junctions _ . . . . . . . . . 112

6.3 Algorithms and Tools for Investigating Device-level Properties . . . 114

6.4 Exploring Ensembles of Devices . . . . . . . . . . . . . . 115

6.5 Characterizing the Accuracy and Limitations of SLSTM _ . . . . 116

References . . . . . . . . . . . . . . . . . . . 117

A Permission to Republish Work from Royal Society of Chemistry 130

B Permission to Republish Work from Cambridge University Press 133

C Permission to Republish Figures from Lee et al. . . . . . . . 135

D Permission to Republish Figures from Savaikar et al. . . . . . 137 


\section{List of Figures}

2.1 Scanning Electron Micrographs of QDs-BNNTs _ . . . . . . . . 12

2.2 Experimental Current-Voltage Characteristics of QDs-BNNTs of Differing Lengths . . . . . . . . . . . . . . . . . . . . . 13

2.3 Schematic of 1D Chain of Gold Nano-Islands Used in MITS Simula-

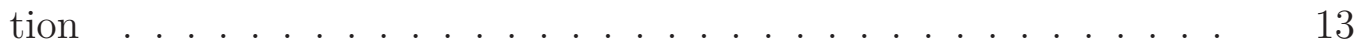

2.4 Simulated Current-Voltage Characteristics of 1D Chain of Gold NanoIslands . . . . . . . . . . . . . . . . . . . . . . . . 18

2.5 Simulated Current-Voltage as a Function of Temperature . . . . . 20

2.6 Schematic of Disordered 2D Devices from MITS Simulations . . . . 22

2.7 Distribution of Current Among Different Paths in 2D Device . . . 23

2.8 Junction Resistances in 200 Junction Device at Different Voltages . 25

3.1 FEM Diagram . . . . . . . . . . . . . . . . . . . 35

$3.2 \quad$ Spherical Capacitor . . . . . . . . . . . . . . . . 40

3.3 Sphere with Impinging Sphere Image . . . . . . . . . . . . . 41

3.4 Sphere with Impinging Sphere Graph . . . . . . . . . . . . . 42

3.5 Multiple Surrounding Spheres Image _. . . . . . . . . . . . . 43

3.6 Multiple Surrounding Spheres Graph . . . . . . . . . . . . 44

3.7 Density of Impinging Spheres _ . . . . . . . . . . . . . . 45

3.8 Electrostatic Potential Around Spheres . . . . . . . . . . . . 46

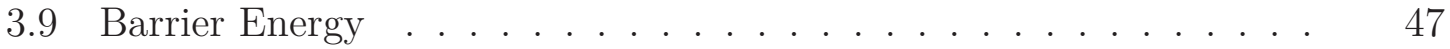

3.10 Capacitance of Linear Chains _. . . . . . . . . . . 50

$3.11 I-V$ Calculations with Analytical and FEM Capacitances . . . . . 51 
3.12 Bent QDs-BNNTs ............................ 53

3.13 Bent QDs-BNNTs Capacitance Distributions . . . . . . . . . 54

3.14 Capacitance of 2D System . . . . . . . . . . . 57

4.1 Current-Voltage for Randomized Resistance Systems . . . . . . . . 61

4.2 Gold Clusters . . . . . . . . . . . . . . . . . 67

4.3 Tunneling Barrier Profile . . . . . . . . . . . . . . 68

4.4 Conductance for $\mathrm{Au}_{32}, \mathrm{Au}_{6}$, and single-atoms . . . . . . . . 69

4.5 Electronic States of Hexagonal System . . . . . . . . . . . 71

4.6 Conductance Vs. Distance for Each Investigated System . . . . . . 72

5.1 Dimer-Adatom-Stacking Fault model . . . . . . . . . . . . . . 82

5.2 Constant current images from experiment and simulations . . . . . 84

5.3 Current imaging tunneling spectroscopy images from experiment and

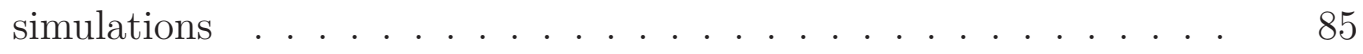

5.4 Badness of fit between SLSTM calculations and experiment . . . . . 86

5.5 Comparison of simulated linescans to experiment . . . . . . . . 87

5.6 Density of States for selected atoms in $\operatorname{Si}(111)-(7 \times 7) \ldots . . . . \quad 88$

5.7 Geometry of $(\mathrm{PbS})_{32}$ baby crystal . . . . . . . . . . . . . 91

5.8 Current and conductance calculations for $(\mathrm{PbS})_{32} \ldots \ldots$. . . . . 93

5.9 Projected density of states $(\mathrm{PDOS})$ for $(\mathrm{PbS})_{32}$ and tips . . . . . 94

$5.10 \mathrm{NDR}$ as an interaction between $(\mathrm{PbS})_{32}$ and tip density of states . . 96

5.11 Differential conductance calculations for $(\mathrm{PbS})_{32}$ with alternate tips $\quad 97$

5.12 Geometry of Si doped BN . . . . . . . . . . . . 100

5.13 Current-Voltage characteristics of Si doped BN . . . . . . . . . 101

5.14 Differential conductance characteristics of Si doped BN . . . . . 103

5.15 Constant current STM images of Si doped BN . . . . . . . . . . 104

5.16 Geometry of $\mathrm{MoS}_{2}$ with attached Au atomic wire . . . . . . . . 107

5.17 Current-Voltage characteristics of $\mathrm{MoS}_{2}$ with attached atomic wires 108 


\section{Preface}

\section{Chapter 2: Simulation of Disordered Coulomb Blockade Devices}

Chapter 2 is a work previously published in Simulation of Charge Transport in Disordered Assemblies of Metallic Nano-Islands: Application to BoronNitride Nanotubes Functionalized with Gold Quantum Dots. John A. Jaszczak, Madhusudan A. Savaikar, Douglas R. Banyai, Boyi Hao, Dongyan Zhang, Paul L. Bergstrom, An-Ping Li, Juan-Carlos Idrobo, And Yoke Khin Yap. MRS Symposium Proceedings Spring 2014, Symposium MM, 2014. [1], copyright 2014 Materials Research Society. Reprinted with permission. See appendix B for documentation of permission to reprint. This work also incorporates results previously published in Lee et al. [2] and Savaikar et al. [3].

JAJ prepared the manuscript. MAS performed the MITS simulations. DRB performed FEM capacitance calculations. BH, DZ, and YKY manufactured the onedimensional QDs-BNNT devices. APL and JCI performed measurements on the QDs-BNNT devices. JAJ, MAS, DRB, BH, PLB, and YKY contributed to the analysis.

\section{Chapter 3: Calculation of Capacitance in Dense Disordered Systems}

All work in Chapter 3 was performed by DRB with the following exceptions:

The geometry of the linear one-dimensional and the two-dimensional devices were created by Dr. M. A. Savaikar (MAS). Current-voltage calculations were performed 
by DRB and MAS using the MITS simulator developed by MAS. Calculations for the bent two-dimensional devices were performed in collaboration with P. K. Hazaveh. Dr. P. L. Bergstrom and Dr. J. A. Jaszczak assisted in analysis.

\section{Chapter 4: Tunneling Resistance Between Clusters}

All work in Chapter 4 was performed by DRB with the following exceptions:

The current-voltage calculations on Coulomb blockade devices was based on work by Dr. M. A. Savaikar. Dr. Ranjit Pati provided the program for calculating currents using the Green's function method. Dr. R. Pandey, Dr. J. Loh, and Dr. J. A. Jaszczak assisted in analysis.

\section{Chapter 5: Simulation of Scanning Tunneling Microscopy and Spec- troscopy}

All work in Chapter 5 was performed by DRB with the following exceptions:

The integration routine at the core of the SLSTM software was originally written by Dr. H. He of Valparaiso University. The calculation of the geometry and electronic structure of the $\mathrm{Si}(111)-(7 \times 7)$ system was performed by Dr. H. He of Valparaiso University.

Section 5.4 is a summary of work published in Electron tunneling characteristics of a cubic quantum dot, $(\mathrm{PbS})_{32}$. Sanjeev K. Gupta, Haiying He, Douglas Banyai, Anil K. Kandalam, and Ravindra Pandey. The Journal of Chemical Physics, 2013. [4]. Manuscript preparation and geometry and electronic structure 
calculations were performed primarily by SKG. DRB calculated STS properties. HH, $\mathrm{AKK}$, and RP assisted in analysis.

Section 5.5 is a summary of work published in Effect of Si doping on the electronic properties of BN monolayer. SAnjeev K. Gupta, Haiying He, Douglas Banyai, Mingsu Si, Ravindra Pandey, and Shashi P. Karna. Nanoscale, 2014. [5]. Manuscript preparation and geometry and electronic structure calculations were performed primarily by SKG. DRB calculated STM topographs and STS properties. HH, MS, RP, and SPK assisted in analysis.

Section 5.6 is a summary of work published in Electronic stability and electron transport properties of atomic wires anchored on the $\mathrm{MoS}_{2}$ monolayer. Ashok Kumar, Douglas Banyai, P. K. Ahluwalia, Ravindra Pandey, and Shashi P. Karna. Phys. Chem. Chem. Phys., 2014. [6]. Manuscript preparation and geometry and electronic structure calculations were performed primarily by AK. DRB calculated STS properties. PKA, RP, and SPK assisted in analysis. 



\section{Acknowledgments}

This work would not have been possible without the generosity, intellect, and patience of a great number of friends, colleagues, and collaborators. I'd like to thank first and foremost my advisor Dr. John Jaszczak, the Department of Physics as lead by Dr. Ravi Pandey, and my family, including, of course, Dr. Amalia Anderson.

I am grateful for the insight of my committee and other faculty members, including Dr. Ravi Pandey, Dr. Loredana Valenzano, Dr. Paul Bergstrom, and Dr. Y. K. Yap, and a great number of fellow graduate students, postdocs, and visiting scholars including Dr. Madhusudan Savaikar, Dr. Haiying He, Dr. Sanjeev Gupta, Dr. Ashok Kumar, Wil Slough, Dr. Gowtham, Dr. Jarvis Loh, Dr. Munima Sahariah, Kevin Waters, and Boyi Hao.

Support was generously provided by the Michigan Tech Department of Physics, the Miles Fellowship, the Graduate School Finishing Fellowship, IASST Guwahati, St. Xavier's College Ahmedabad, Himachal Pradesh University, and Panjab University. 



\section{Abstract}

For half a century the integrated circuits (ICs) that make up the heart of electronic devices have been steadily improving by shrinking at an exponential rate. However, as the current crop of ICs get smaller and the insulating layers involved become thinner, electrons leak through due to quantum mechanical tunneling. This is one of several issues which will bring an end to this incredible streak of exponential improvement of this type of transistor device, after which future improvements will have to come from employing fundamentally different transistor architecture rather than fine tuning and miniaturizing the metal-oxide-semiconductor field effect transistors (MOSFETs) in use today.

Several new transistor designs, some designed and built here at Michigan Tech, involve electrons tunneling their way through arrays of nanoparticles. We use a multi-scale approach to model these devices and study their behavior. For investigating the tunneling characteristics of the individual junctions, we use a first-principles approach to model conduction between sub-nanometer gold particles. To estimate the change in energy due to the movement of individual electrons, we use the finite element method to calculate electrostatic capacitances. The kinetic Monte Carlo method allows us to use our knowledge of these details to simulate the dynamics of an entire devicesometimes consisting of hundreds of individual particles - and watch as a device 'turns on' and starts conducting an electric current.

Scanning tunneling microscopy (STM) and the closely related scanning tunneling spectroscopy (STS) are a family of powerful experimental techniques that allow for the probing and imaging of surfaces and molecules at atomic resolution. However, interpretation of the results often requires comparison with theoretical and computational models. We have developed a new method for calculating STM topographs 
and STS spectra. This method combines an established method for approximating the geometric variation of the electronic density of states, with a modern method for calculating spin-dependent tunneling currents, offering a unique balance between accuracy and accessibility. 


\section{Chapter 1}

\section{Introduction}

A significant area of active research is in the development of modern electronic devices and the fabrication methods that make them possible. The principle component of most electronics is the field-effect transistor (FET), specifically the metal-oxidesemiconductor FET (MOSFET). MOSFET technology is incredibly mature, and further improvements are becoming increasingly more expensive to develop. This helps motivate the development of different classes of transistors that have the possibility of replacing MOSFETs in very-large-scale (VLSI) integrated circuits. One such device is the single-electron transistor (SET). Whereas miniaturization of MOSFETs is

limited to a large degree by the quantum mechanical tunneling of electrons through the thin insulating layers required by these smaller devices, SETs would not function without this tunneling process. Understanding the tunneling process in these devices is fundamental to understanding and perhaps improving the behavior of the devices.

The scanning tunneling microscope (STM) is another system defined by the tunneling process. The STM is commonly used to image surfaces at atomic resolution. Additionally, it can be used to probe the local electronic structure of materials and defects 
in materials, and can even be used to manipulate individual atoms.

In this work, we investigate electron transport in these systems, namely single-electron transistors and scanning tunneling microscopes. The transport is modeled at several levels of theory and at multiple scales. Several computational tools are developed to aid in this modeling, and are described within.

\subsection{Modern Electronics}

\subsubsection{The Perpetually Impending End of Moore's Law}

For half a century the integrated circuits that make up the heart of nearly all electronic devices have been improving at an exponential rate. This trend was famously described in a 1965 paper by Gordon Moore [7]. Specifically Moore noted that the

"complexity for minimum component costs" was roughly doubling every year. The component density of integrated circuits (ICs) has been steadily increasing ever since, and the direct effect is now ICs with billions of transistors are commonplace. Additionally, as ICs are made more dense the speed of operation increases and the energy consumed decreases.

These improvements in ICs are not just about making the things we already have better. Novel technologies are enabled by the dramatic decrease in size and power consumption. Examples include ingestible sensor systems (electronic pills) that can make measurements of the digestive tract [8] or make targeted deliveries of drugs [9].

However, no exponential growth can continue forever, and the end of Moore's law has been foretold ever since it was first described [10]. The current method for producing 
ICs depends on photolithography to selectively dope, etch, and deposit progressive layers on top of a silicon substrate. The current state of the art produces transistors that are on the order of $(100 \mathrm{~nm})^{2}$ in area with $14 \mathrm{~nm}$ wide features and gate insulating layers that are only a few nm thick. As devices get smaller and these insulators become thinner, electrons leak through due to quantum mechanical tunneling. This seems to be a fundamental issue that places a hard lower limit on transistor size, and may be the downfall of Moore's law.

\subsubsection{New Transistor Designs Use Tunneling to Their Ad- vantage}

While ICs will no doubt continue to improve, we will eventually see these improvements come from employing fundamentally different transistor architecture rather than fine tuning and miniaturizing the field-effect transistors in use today. One of the most interesting 'new' architectures is the single-electron transistor (SET). The idea behind SETs is old $[11,12]$, and functioning SETs have been demonstrated as early as 1987 [13]. However, these early devices only operated at liquid helium temperatures, and it has only been more recently that devices capable of room temperature operation have been fabricated $[2,14-16]$. New designs for SETs that will work at room temperature and can be built at scale is an area of active research $[16,17]$.

The simplest SET design consists of an isolated 'island' electrode connected by tunnel junctions to source and drain electrodes, and capacitively coupled to a gate electrode. In order for such a device to work at room temperature, the island needs to be smaller than $\sim 10 \mathrm{~nm}$. Reliably creating such precise devices is beyond current manufacturing capabilities. For example, it is estimated the current state of the art $22 \mathrm{~nm}$ photolithography could produce islands on the order of $~ 100 \mathrm{~nm}$. 


\subsubsection{Randomly Created Devices with Randomized Proper- ties}

Another approach for creating room temperature SETs is the many-island SET (MISET), where there are several to thousands of islands between the source and drain electrodes. An advantage of this approach is it is possible to create the source, drain, and gate electrodes using standard lithographic procedures and then to fill in the relatively large source-drain gap with a sea of nanoislands. Some groups have used thiolated gold nanoparticles for the nanoislands [18]. Dr. Bergstrom's group has used a focused ion beam (FIB) to deposit a disordered film of metallic nanoparticles [17]. Dr. Yap's group has taken a different approach using pulsed laser deposition to deposit metallic nanoparticles onto an insulating boron nitride nanotube [2].

\subsubsection{Simulation of SETs}

At a high level, we model the conduction process in many-island SETs (MISETs) using a semi-classical model, sometimes referred to as "orthodox theory" [19, 20]. This allows us to calculate the effect that charging energies have on the rates of tunneling events. These charging energies are calculated from the capacitances in the system. To more accurately calculate these energies, we built a system to calculate the capacitances using the finite element method (FEM). The tunneling rates depend on the tunneling conductances. To investigate tunneling conductances, we calculated conductances using density functional theory (DFT). 


\subsection{Scanning Tunneling Microscopy and Spec- troscopy}

Scanning tunneling microscopy (STM) has become an essential tool for nanoscale science and engineering since its development in 1981 [21]. A scanning tunneling microscope consists primarily of a metallic probe with an atomically fine tip, piezo-

electric actuators to move the tip with sub- $\AA$ precision, electronics to measure currents through the tip and control the positioning of the tip, and a computer to process the resulting data into 2D or 3D images of the measured surface. The tunneling process is incredibly sensitive to the barrier width, which in this case is the vacuum distance between the tip and the sample. This allows for measuring surface heights with sub- $\AA$ precision.

The STM has many possible modes of operation, with the conceptually simplest being constant height imaging (CHI), and the most common being constant current imaging (CCI). In constant height imaging, the tip is scanned across the surface at a constant height and with a constant bias relative to the grounded sample, and the tunneling current is measured as a function of position. In constant current mode, the height of the tip is adjusted to maintain a preset tunneling current while the tip is scanned across the surface. Another interesting mode is current imaging tunneling spectroscopy (CITS). In this mode, the control electronics switch rapidly between CCI mode, where the tip height is adjusted to maintain a set current, and a spectroscopy mode where the tip position is held fixed and the current is measured as a function of applied bias. Such scanning tunneling spectroscopy (STS) measurements reveal information about the energy dependence of the electronic density of states, $\rho(E)$. 


\subsection{Dissertation Outline}

The primary thrust of this dissertation is to model the electron transport in large single electron devices (SEDs) at a more advanced level than has been done before, in order to explore the complicated behaviors that arise in many-island SEDs. In chapter 2, we investigate the conductance characteristics of one- and two-dimensional MISETs [1], motivated to a large degree by the experimental work performed at Michigan Tech by the research groups of Dr. Paul Bergstrom and Dr. Yoke Khin Yap. We use a semi-classical model for tunneling combined with a kinetic Monte Carlo method for integrating these rates and deriving observable properties, as implemented in our newly developed Multi-Island Transport Simulator (MITS) code [3]. We investigate effects of disorder, device length, and temperature on $1 \mathrm{D}$ devices. In some $2 \mathrm{D}$ devices we find a robust dominant conducting path (DCP), allowing these devices to be viewed as effective $1 \mathrm{D}$ devices.

Chapter 3 describes efforts made to improve our device modeling abilities by improving our method for calculating capacitances. We developed a software package that utilizes the SALOME [22] computer aided engineering (CAE) software package and the Elmer [23] finite element method (FEM) solver to calculate capacitances for a device consisting of an arbitrary arrangement of spherical islands. We investigate the effect of neighbor islands on the capacitances of an individual island through a set of test calculations, and we compare MITS calculations that use analytic capacitance calculations to calculations using our FEM capacitance calculations. These numerical capacitance calculations are employed in a MITS study of bent 1D devices.

In our studies of MISETs it became apparent that our treatment of the tunneling resistance between neighboring islands warrants some deeper inspection. The tunneling resistance is one of the primary inputs to the MITS model, and, as was found to be 
the case for the capacitances, could be expected to be influenced by the detailed geometry of the structures. In chapter 4 we investigate tunneling between nanoparticles using a density functional theory (DFT) approach, and make comparisons to analytic tunneling calculations. This is a preliminary investigation, and ideas for furthering the work can be found in chapter 6 .

While working with the research group of Dr. Pandey for the above mentioned DFT studies, an interesting opportunity for collaboration appeared regarding tunneling away from surfaces, in the form of scanning tunneling microscopy measurements. Chapter 5 discusses the fruits of this collaboration, including a newly developed STM/STS simulation tool that balances accuracy and accessibility, and several published journal articles making use of the tool. This chapter begins with a review of established methods for simulating scanning tunneling microscopy results, and then describes a new method we developed. The method is validated by simulating the well-known $\mathrm{Si}(111)-(7 \times 7)$ reconstructed surface. The method is also applied to studies of $(\mathrm{PbS})_{32}$ nanoclusters [4], silicon-doped boron nitride monolayers [5], and atomic wires on $\mathrm{MoS}_{2}$ monolayers [6].

Finally, in chapter 6 we provide a summary of the work described within, and provide an outlook on interesting and important avenues for future work. 



\section{Chapter 2}

\section{Simulation of Disordered Coulomb Blockade Devices ${ }^{1}$}

\subsection{Abstract}

In this study, we investigate the charge-transport behavior in a disordered onedimensional (1D) chain of metallic islands using the newly developed multi-island transport simulator (MITS) based on semi-classical tunneling theory and kinetic Monte Carlo simulation. The 1D chain is parameterized to model the experimentallyrealized devices studied by Lee et al. [Advanced Materials 25, 4544-4548 (2013)], which consists of nano-meter-sized gold islands randomly deposited on an insulating boron-nitride nanotube. These devices show semiconductor-like behavior without having semiconductor materials. The effects of disorder, device length, temperature, and source-drain bias voltage $\left(V_{s d}\right)$ on the current are examined. Preliminary results of random assemblies of gold nano-islands in two dimensions (2D) are also examined

\footnotetext{
${ }^{1}$ The material contained in this chapter was previously published in [1]
} 
in light of the $1 \mathrm{D}$ results.

At $T=0 \mathrm{~K}$ and low source-drain bias voltages, the disordered 1D-chain device shows charge-transport characteristics with a well-defined Coulomb blockade (CB) and Coulomb staircase (CS) features that are manifestations of the nanometer size of the islands and their separations. In agreement with experimental observations, the $\mathrm{CB}$ and the blockade threshold voltage $\left(V_{t h}\right)$ at which the device begins to conduct increases linearly with increasing chain length. The CS structures are more pronounced in longer chains, but disappear at high $V_{s d}$. Due to tunneling barrier suppression at high bias, the current-voltage characteristics for $V_{s d}>V_{t h}$ follow a non-linear relationship. Smaller islands have a dominant effect on the $\mathrm{CB}$ and $V_{t h}$ due to capacitive effects. On the other hand, the wider junctions with their large tunneling resistances predominantly determine the overall device current. This study indicates that smaller islands with smaller inter-island spacings are better suited for practical applications. Temperature has minimal effects on high-bias current behavior, but the CB is diminished as $V_{t h}$ decreases with increasing temperature.

In $2 \mathrm{D}$ systems with sufficient disorder, our studies demonstrate the existence of a dominant conducting path (DCP) along which most of the current is conveyed, making the device effectively quasi-1-dimensional. The existence of a DCP is sensitive to the device structure, but can be robust with respect to changes in $V_{s d}$.

\subsection{Introduction}

Recent advances in the development of new materials and fabrication techniques have spurred continued interest in further miniaturization of conventional field-effect devices with new device structure designs [24]. Multi-gate architectures have been 
fabricated that may allow further reduction in the dimensions of classical metal-oxide semiconductor field effect transistors (MOSFET) without degrading the transistor performance [25]. On the other hand, conduction by tunneling in granular metallic systems has been a subject of interest for many years [26, 27]. Single-electron transport devices that operate based on tunneling of individual electrons through junctions formed with one or more nanometer-sized islands have been demonstrated, some even operating at room temperature [16, 28]. Successful attempts have been made to demonstrate their use as single-electron memory devices and for nanometerscale displacement sensing [29, 30].

This computational study attempts to complement experimental work seeking to elucidate the effects of different factors such as structural disorder on electron tunneling transport $[2,18]$ by beginning to systematically explore the effects of island sizes, inter-island spacings, and conduction channel length on $I-V$ characteristics [3]. Particular focus is given to modeling charge transport in boron-nitride nanotubes (BNNTs) functionalized with nanometer-size gold islands. The device properties are investigated at low and high biases, and the effects of temperature on the Coulomb blockade and the device threshold voltage are studied. Later, the work is further extended to study the effect of structural disorder on 2D device characteristics that gives an insight into the functioning of experimentally fabricated multi-dimensional devices.

Functionalization of high-quality 20-80 nm diameter BNNTs with gold quantum dots deposited by pulsed laser deposition has recently been demonstrated by Lee et al. [2] (figure 2.1). Without gold-dot functionalization, the BNNTs are excellent insulators, and show currents of less than $10^{-11} \mathrm{~A}$ under bias potentials up to $180 \mathrm{~V}$. On the other hand, the gold quantum-dot-functionalized BNNTs (QDs-BNNTs) exhibit room temperature semiconductor-like switching behavior, with turn-on voltages $\left(V_{t h}\right)$ in the range of 2.0 to $34.0 \mathrm{~V}$, increasing with increasing length (L) of the QDs-BNNT device, 

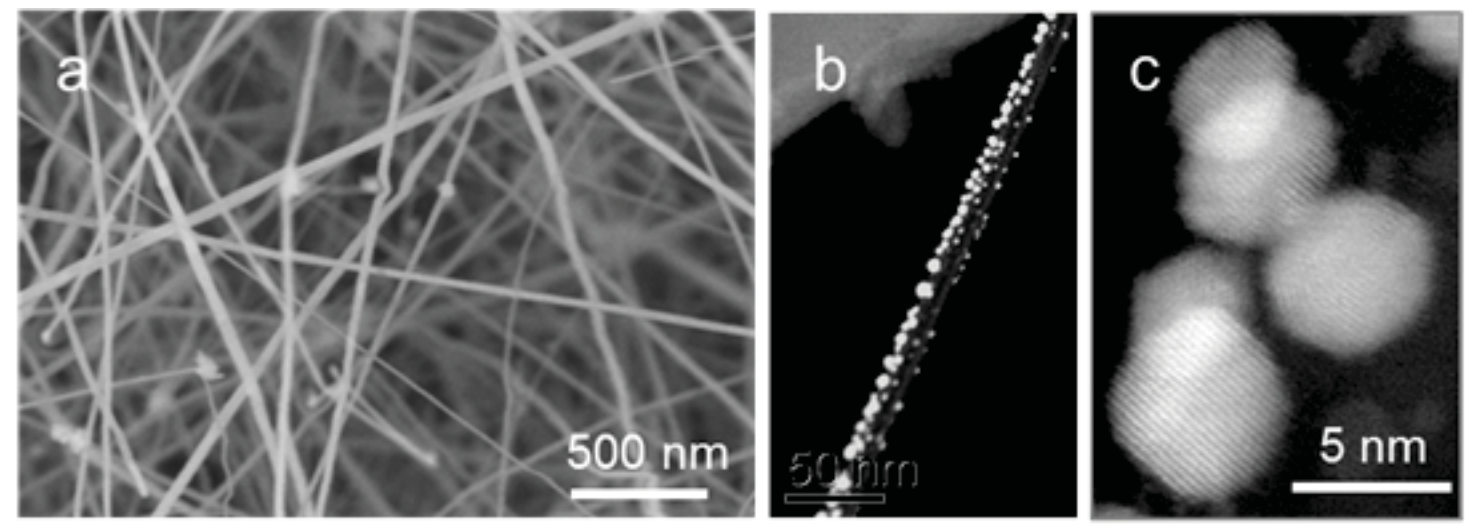

Figure 2.1: Images of gold quantum dot functionalized boron nitride nanotubes (QDs-BNNTs) obtained by (a) scanning electron microscopy and $(\mathrm{b}, \mathrm{c})$ scanning transmission electron microscopy. Reprinted with permission from Lee et al. [2]. Copyright (0) 2013 WILEY-VCH Verlag GmbH \& Co. KGaA, Weinheim. See appendix $\mathrm{C}$ for documentation of permission to republish this material.

where L ranges from 1.29 to $2.37 \mu \mathrm{m}$ (figure 2.2).

\subsection{Theory and Simulation}

Initial investigations were focused on model one-dimensional systems corresponding to the QDs-BNNTs of Ref. [2] (figure 2.3). The model device consists of a chain of 199 gold islands (200 junctions) between source and drain electrodes. In this study, the BNNT is assumed to play no role other than to geometrically align the islands because of its insulating nature in the absence of gold islands. The radius of each island is randomly selected from a uniform distribution between 3 and $10 \mathrm{~nm}$, while the junction widths are randomly chosen from a uniform distribution in the range of 1 and $5 \mathrm{~nm}$. An island at one end of the chain is selected to be a fixed drain electrode, while the source (ground) electrode is chosen from among the remaining islands in the chain, thus fixing the number of islands in the system (chain) and its length. 


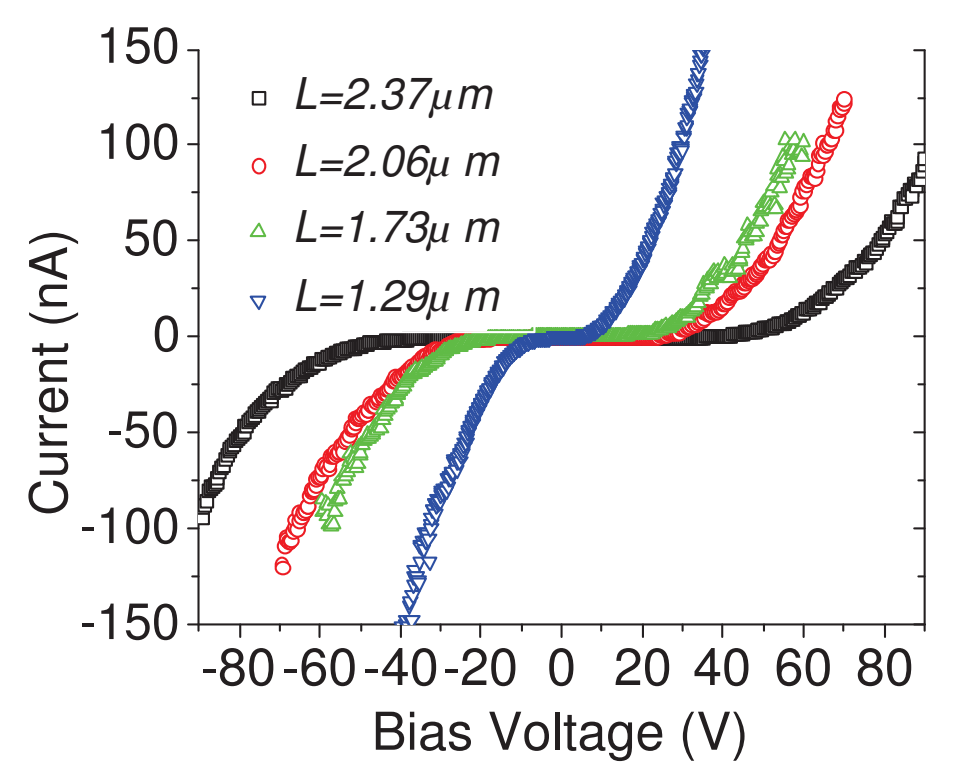

Figure 2.2: Current-voltage characteristics of QDs-BNNT devices of different lengths demonstrating non-Ohmic behavior, and Coulomb-blockade effects. Data collected using 4-probe scanning tunneling microscopy. Reprinted with permission from Lee et al. [2]. Copyright (C) 2013 WILEYVCH Verlag GmbH \& Co. KGaA, Weinheim. See appendix C for documentation of permission to republish this material.

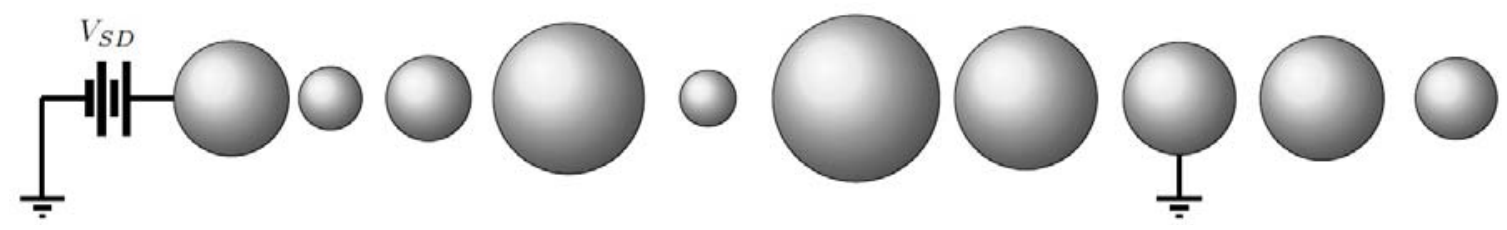

Figure 2.3: Schematic of the geometrical model of a $1 \mathrm{D}$ chain of gold nanoislands with randomly selected island radii and junction widths, deposited on an insulating born-nitride nanotube used for the MITS simulation of systems fabricated by Lee et al. [2]. Reprinted with permission from Savaikar et al. [3]. Copyright 2013, AIP Publishing LLC. See appendix D for documentation of permission to republish this material.

Conduction in the multi-island devices is modeled using kinetic Monte Carlo simulation methods $[31,32]$ based on tunneling rates that are computed semi-classically (see Ref. [3] for further details). The probabilities for tunneling between any pair of nearby islands at any given time depend on three primary factors: the charge states of the islands, the voltage drops across the junctions, and the junctions' tunneling 
resistances. All three of these factors can dynamically vary during the simulation. In particular, in contrast to most models that use tunneling resistances that are fixed throughout the simulation, the tunneling resistances in MITS dynamically vary with the voltage drops across the junctions, both due to the applied voltage bias, and the charge states of the capacitively coupled islands.

The semi-classical approach used for calculating the tunneling rates assumes that (i) the energy spectrum of the conductive islands may be considered continuous (ii) the tunneling time is negligible compared to the time between successive tunneling events, and (iii) coherent tunneling events are ignored [20,33]. For a pair of adjacent islands $\mathrm{i}$ and $\mathrm{j}$, the tunneling rate is given by $[19,20,33]$

$$
\Gamma_{i, j}\left(\Delta W_{i, j}\right)=\left(\frac{-\Delta W_{i, j}}{e^{2} R_{i, j}}\right)\left[1-\exp \left(\frac{\Delta W_{i, j}}{k_{B} T}\right)\right]^{-1},
$$

where $\Delta W_{i, j}$ is the change in the free energy of the system due to the tunneling event, $R_{i, j}$ is the tunneling resistance of the junction, e is the electron charge, $k_{B}$ is the Boltzmann constant, and $T$ is the temperature. As is clear from Eq. 2.1, $\Delta W_{i, j}$ and $R_{i, j}$ each play key roles in determining the tunneling rates across the device.

Consider first the change in free energy due to the transition, which is given by $\Delta W_{i, j}=-e V_{i, j}+E_{c i, j}$. This depends on the potential drop $V_{i, j}$ that exists across the junction before the transition. $V_{i, j}$ in turn depends on the capacitances of the system, which are fixed, and the charge state of the system, which dynamically evolves. The junction charging energy, $E_{c i, j}$, is the energy required for a single electron to tunnel across the junction between the two uncharged coupled islands, $\mathrm{i}$ and $\mathrm{j}$, and depends on all of the capacitances of the system [19, 20, 33]. An analytical method employing image charges was used for the calculation of junction capacitances $C_{i, j}$ between neighboring islands $[34,35]$, and the dielectric constant of the junction material was 
taken simply to be 1. Given the self-capacitances and junction capacitances, a capacitance matrix $\mathbf{C}$ is constructed that relates $\vec{Q}$, a vector composed of the charges on the islands, and $\vec{V}$, a vector composed of the island potentials through the matrix equation $\vec{Q}=\mathbf{C} \vec{V}[33]$. As the charge state $\vec{Q}$ of the device changes, the matrix equation is used to solve for the island potentials.

The tunneling rate for a junction is also dependent on the junction's tunneling resistance $R_{i, j}$, which is a strong function of the device geometry as it increases exponentially with the fixed junction separation $d_{i, j}$. $R_{i, j}$ also depends strongly on the height of the energy barrier between the two islands that form the junction. The barrier height depends on the work function of the islands $\varphi$, as well as the potential drop $V_{i, j}$ across them. If $e V_{i, j}$ remains small compared to $\varphi$, it has a negligible effect on the barrier height and $R_{i, j}$ would be a constant. Under simulation conditions in which all the junction resistances in a given chain remain constant, the device $I-V$ characteristics follow a linear behavior for large source-drain voltage biases. However, under high bias conditions, especially where there is a large charge build on some islands, the potential difference between the neighboring islands can be significant compared to $\varphi$, leading to significant band bending. As a result, the effective barrier height would strongly depend on $V_{i, j}$ and subsequently, $R_{i, j}$ would vary significantly with the applied source-drain bias or with the charge state during the course of the simulation. Although a junction's barrier height decreases approximately linearly from one island to the next, in order to simplify the calculations, the tunneling barrier is taken to be of constant height across the width of the junction, but with a reduced height whose variation is given by $\varphi_{e f f_{i j}}=\varphi-e V_{i, j} / 2$, a reasonable approximation as long as for each junction $V_{i, j}<\varphi[36]$. Thus the tunneling resistances are given by $[27]$

$$
R_{i, j}=\left(\frac{h^{3}}{64 \pi^{2} m_{e} e^{2}}\right)\left(\frac{E_{f}+\varphi_{e f f_{i, j}}}{E_{f}}\right)\left(\frac{\exp \left(2 \alpha k_{0} d_{i, j}\right)}{\varphi_{e f f_{i, j}}}\right)\left(\frac{\alpha k_{0}}{r_{a}}\right)\left(\frac{1}{G_{i, j}}\right)
$$


where $k_{0}=(2 \pi / h)\left(2 m_{e} \varphi_{e f f_{i, j}}\right)^{\frac{1}{2}}, h$ is the Planck constant and $m_{e}$ is the free electron mass. $\alpha$ is an enhancement parameter that was taken to be 0.115 to set an overall current scale comparable to that measured by Lee et al. [2]. Approximate values of $E_{f}$ and $\varphi$ for gold have been chosen as $5.5 \mathrm{eV}$ and $4.8 \mathrm{eV}$, respectively. The average radius of the two spherical islands forming the junction is $r_{a}$, and $d_{i, j}$ is the closest distance between their surfaces (the junction width). $G_{i, j}$ is a purely geometrical factor that takes into account the solid angle subtended by one spherical island at the other across the tunnel junction when considering the current flux [27].

Simulations were carried out using a newly-developed set of MATLAB ${ }^{\circledR}$-based codes called MITS (Multi-Island Transport Simulator) that is described in detail in Ref. [3]. Important features of MITS include the following:

$\dagger$ The system is described by a physical model of islands and electrodes, in contrast to using fixed resistances and capacitances in a circuit model.

$\dagger$ The model is applicable from low to reasonably high $V_{s d}$. Tunneling barrier heights dynamically change with charge state and $V_{s d}$.

$\dagger$ All islands within a set proximity limit are capacitively coupled to each other.

To begin a simulation, a physical model of a tunneling device is constructed, consisting of spherical metallic islands arranged in one- or two-dimensions, with desired sizes and spacings. For the modeling of the one-dimensional (1D) QDs-BNNT systems, the capacitances are calculated analytically. For two-dimensional (2D) systems, a finite-element-method of calculating the capacitances has been developed in order to account for the important polarization effects of the metallic islands. The circuitmatrix solver builds the capacitance matrix, by which the charging energies for the transfer of a single electron are calculated across all the junctions in a given chain 
$[19,20,33]$. With the given (fixed) electrode potentials and the known island charges (taken to be zero in the initial system configuration), the capacitance matrix is then used to determine the island potentials. The tunneling resistance solver computes the $R_{i, j}$ across all the nearest-neighbor junctions. Once all of the relevant parameters in the system are determined, tunneling rates across the junctions are computed. Following the kinetic Monte Carlo method, a particular tunneling event is randomly selected from among the available events, the corresponding transition is carried out, and the time is updated. Using the system's new charge configuration, the potential drops, the tunneling resistances, and the tunneling rates across all the junctions are recalculated, and the process is repeated for large number of time steps until the current through the device reaches a steady state with satisfactory statistical accuracy.

\subsection{Results}

\subsubsection{One-dimensional devices}

Current-voltage $(I-V)$ characteristics at $\mathrm{T}=0 \mathrm{~K}$ for the model $1 \mathrm{D}$ device are shown in figure 2.4. At high biases (figure 2.4a) the $I-V$ characteristics are non-Ohmic and vary as $I \propto\left(V_{s d}-V_{t h}\right)^{\zeta}$. The exponent $\zeta$ is non-universal and varies between 1 and 3 , increasing from 1 with decreasing chain length. For a fixed $N, \zeta$ also shows a crossover from a lower value at low bias to a higher value at high bias. The Coulomb blockades and Coulomb staircase (CS) structures are shown for different device lengths in figure 2.4b. The blockade width and associated threshold voltage $V_{t h}$ increase with increasing N. The CS structures are also more pronounced for longer devices. 

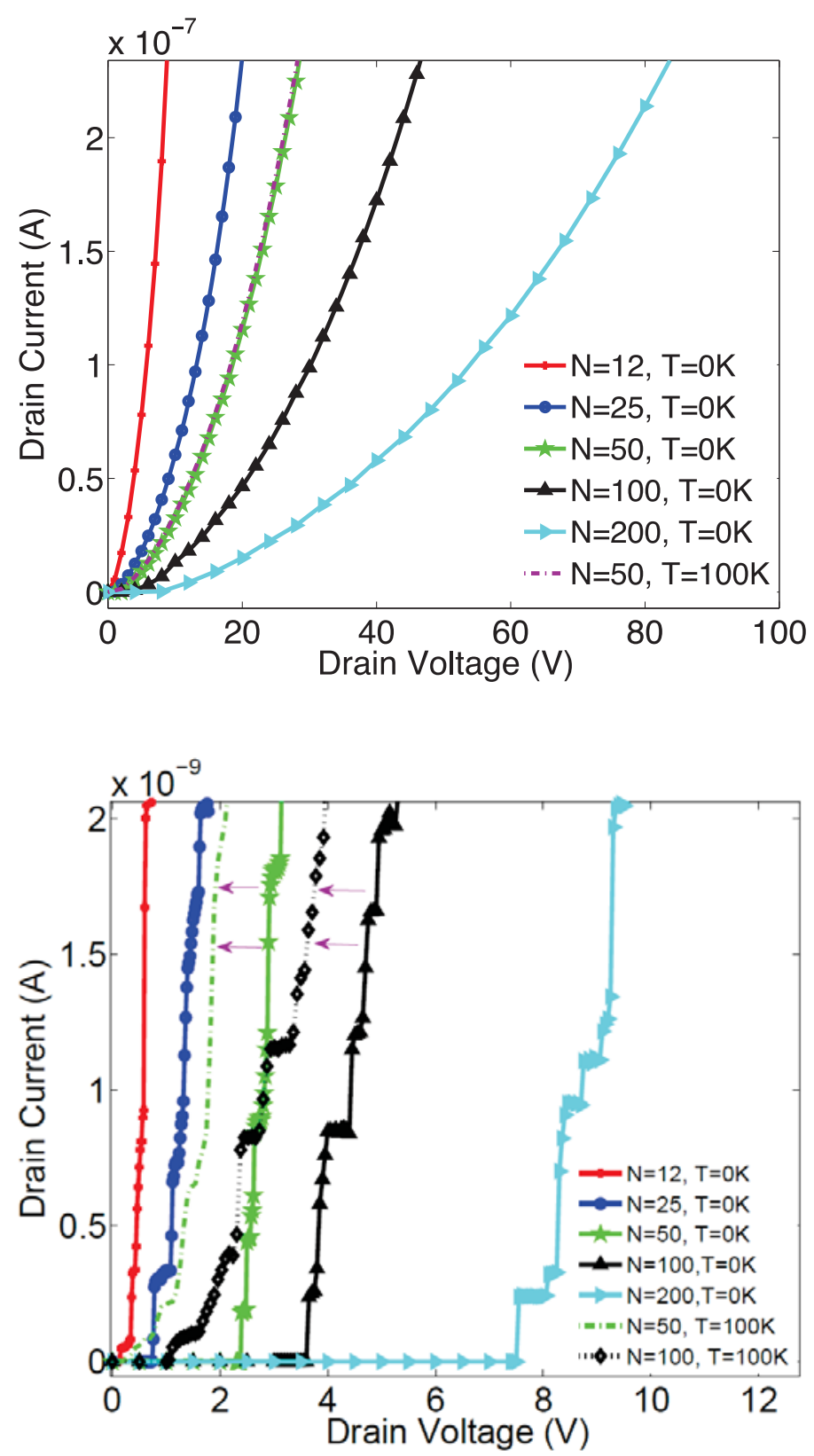

Figure 2.4: Simulated $I-V$ characteristics for the $1 \mathrm{D}$ chain of gold islands as a function of $\mathrm{N}$, the number of islands in between the source and drain electrodes at $\mathrm{T}=0 \mathrm{~K}$. High bias results in (a), which also shows results for $\mathrm{N}=50$ at $\mathrm{T}=100 \mathrm{~K}$. Low bias results are shown in (b), highlighting the Coulomb Blockade and Coulomb staircase structures at $\mathrm{T}=0 \mathrm{~K}$ and 100 K. Reprinted with permission from Savaikar et al. [3]. Copyright 2013, AIP Publishing LLC. See appendix D for documentation of permission to republish this material. 
The effects of temperature on the $I-V$ characteristics are illustrated in figure 2.5. As shown in figure 2.5a, as the temperature is increased, the apparent threshold voltage drops, the Coulomb blockade structure seems to wash out, and the current increases at any given $V_{s d}>V_{t h}$. Figure 2.5b illustrates the effects of temperature on the $I-V$ characteristics of a 25-junction device. At low temperatures, the $\mathrm{T}=0 \mathrm{~K}$ turnon threshold $\left(V_{t h} \approx 0.74 \mathrm{~V}\right)$ and the Coulomb staircases become rounded. As the temperature is increased, the apparent turn-on threshold voltage, the source-drain bias at which the current reaches some minimum detectable level, decreases. For $T \geq 40 \mathrm{~K}$, however, additional Coulomb staircase structures manifest themselves at voltages below the $\mathrm{T}=0 \mathrm{~K}$ threshold voltage. For example, at $\mathrm{T}=40 \mathrm{~K}$ a plateau develops in the current for drain voltages between $\sim 0.35 \mathrm{~V}$ and $0.55 \mathrm{~V}$. The currents associated with the plateaus of the Coulomb staircase steps also increase in magnitude with increasing temperature, while their widths correspondingly decrease.

\subsubsection{Two-dimensional devices}

Experimental studies of $2 \mathrm{D}$ systems show $I-V$ characteristics with similar features to those observed in the 1D systems shown above, including a Coulomb blockade, Coulomb staircases, and non-linear $I-V$ relationships in the "on" state [27, 37-42]. Preliminary investigations of two-dimensional (2D) random arrays of metallic nanoscale islands were carried out using MITS, and are briefly presented here in order to give a view of capabilities for future work.

Simulations of the 2D systems were carried out on a system of 67 spheres, each of

diameter $6.5 \mathrm{~nm}$. Positions of the islands were randomized using Metropolis Monte Carlo, which after decreasing all island diameters to $5.0 \mathrm{~nm}$, resulted in a distribution of nearest-neighbor inter-island spacings ranging between $\sim 1.5$ to $5 \mathrm{~nm}$ (figure 2.6 ), 

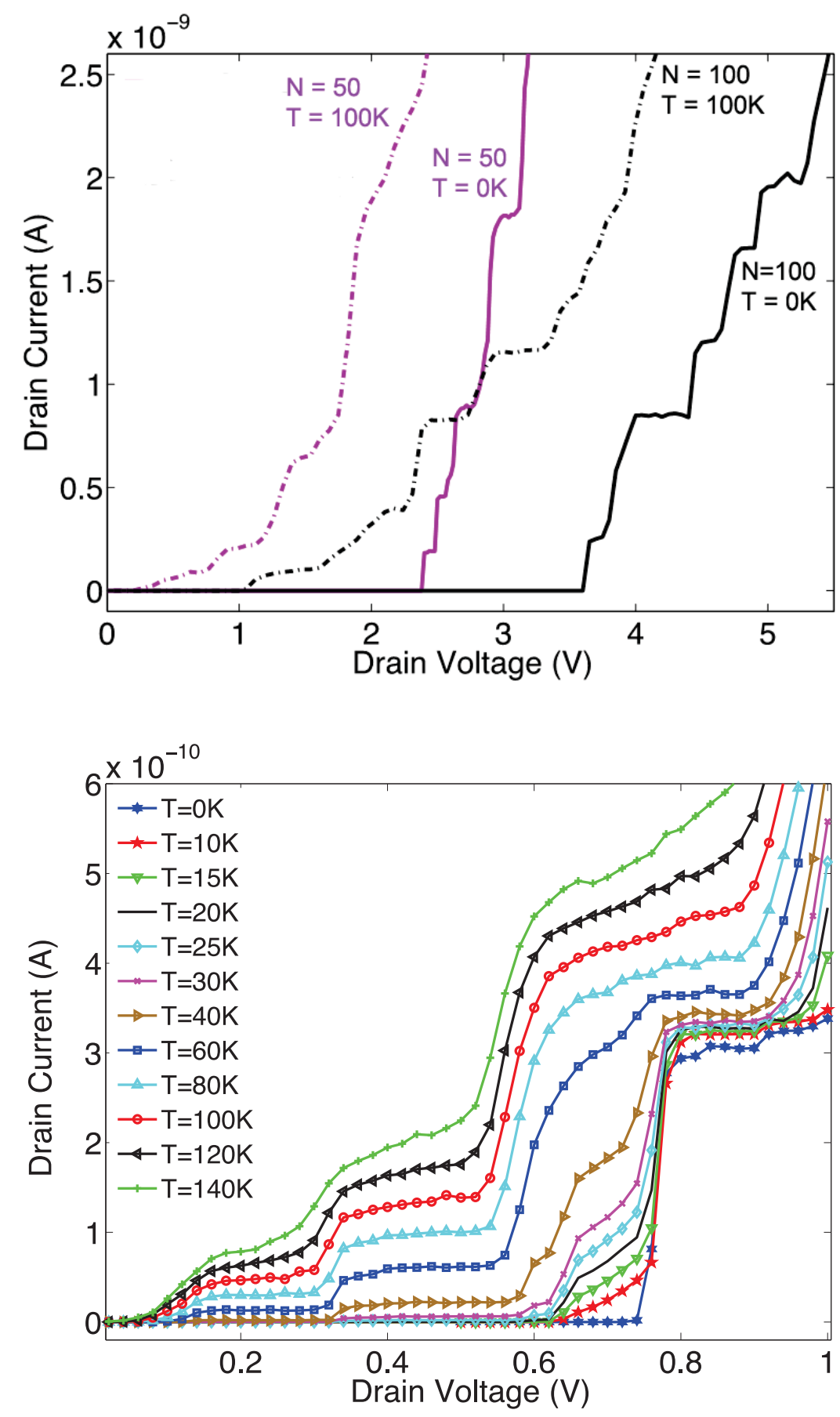

Figure 2.5: Simulated $I-V$ characteristics for a $1 \mathrm{D}$ chain as a function of temperature. (a) $I-V$ characteristics for two different chain lengths at $\mathrm{T}=$ 0 and $\mathrm{T}=100 \mathrm{~K}$. (b) $I-V$ characteristics for a device with $\mathrm{N}=25$ junctions at a series of temperatures between 0 and $140 \mathrm{~K}$. 
and an average spacing of $2.7 \pm 0.1 \mathrm{~nm}$. Simulations were carried out using MITS in the same manner as described above except that the island capacitances and junction capacitances were computed using finite-element methods. ${ }^{2}$ The junction capacitances between near-neighbor islands ranged between $1.5 \times 10^{-20}$ to $10 \times 10^{-20} \mathrm{~F}$. Due to shielding effects from neighboring metallic islands, the island self-capacitances ranged from $0.6 \times 10^{-20}$ to $4.0 \times 10^{-20} \mathrm{~F}$.

As shown in figure 2.6, currents tend to flow in the random $2 \mathrm{D}$ systems along a fairly narrow dominant conducting path, with many junctions carrying greater than $60 \%$ of the total current that is carried to the drain. With increasing $V_{s d}$ (figure 2.6b) the DCP remained relatively robust, and some junctions in the DCP even increase the fraction of the current they carry. At low but non-zero temperatures (figure 2.6c), the DCP also remains robust.

The $I-V$ characteristic for the 67 -island $2 \mathrm{D}$ device is shown in figure 2.7 for sourcedrain biases up to $2 \mathrm{~V}$. The device shows a threshold voltage at $\sim 1 \mathrm{~V}$, and also a weak Coulomb staircase structure compared with the 1D devices. The inset in figure 2.7 shows the currents as a function of $V_{s d}$ for each individual junction in the DCP. These $I-V$ curves show weak Coulomb staircase structures reminiscent of the total device $I-V$ behavior, as one might expect for a junction in a DCP.

\subsection{Discussion}

At any fixed $V_{s d}$ in the on-state, the 1D devices show decreasing currents with increasing device length, as might be expected due to the increased overall resistance of the longer devices and associated increased number of resistive junctions. However,

\footnotetext{
${ }^{2}$ See chapter 3 for details of the finite element method capacitance calculations.
} 

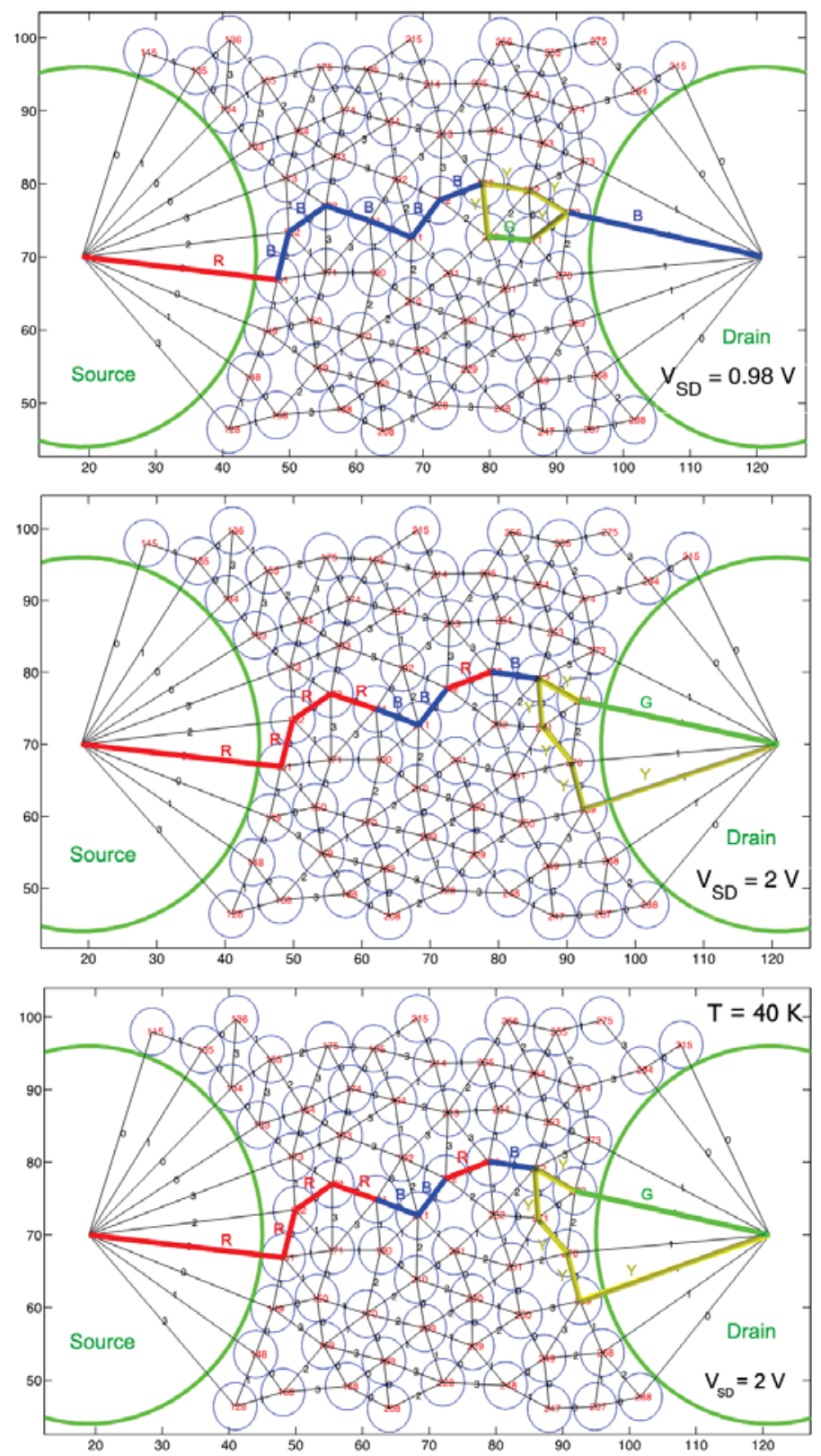

Figure 2.6: Schematic of a disordered 2D device consisting 67 islands, each of radius of $2.5 \mathrm{~nm}$, randomly positioned on the plane between the source and the drain electrodes (large green ellipses) separated by $\sim 50 \mathrm{~nm}$. The nearest neighbor inter-island spacings range anywhere from $\sim 1.5-5 \mathrm{~nm}$. Allowed current paths are shown as solid black line segments. Junctions carrying significant current are color coded according to the percent of the total current carried to the drain: red $(\mathrm{R})=80-100 \%$, blue $(\mathrm{B})=60-80 \%$, green $(\mathrm{G})=40-60 \%$, yellow $(\mathrm{Y})=20-40 \%$. At $\mathrm{T}=0 \mathrm{~K}$ as $V_{s d}$ varies from $0.98 \mathrm{~V}$ (a) to $2 \mathrm{~V}$ (b), the DCP varies but largely retains its dominant conducting nature and position in the $2 \mathrm{D}$ array. (c) The same system at $V_{s d}$ $=2 \mathrm{~V}$ and $\mathrm{T}=40 \mathrm{~K}$. 


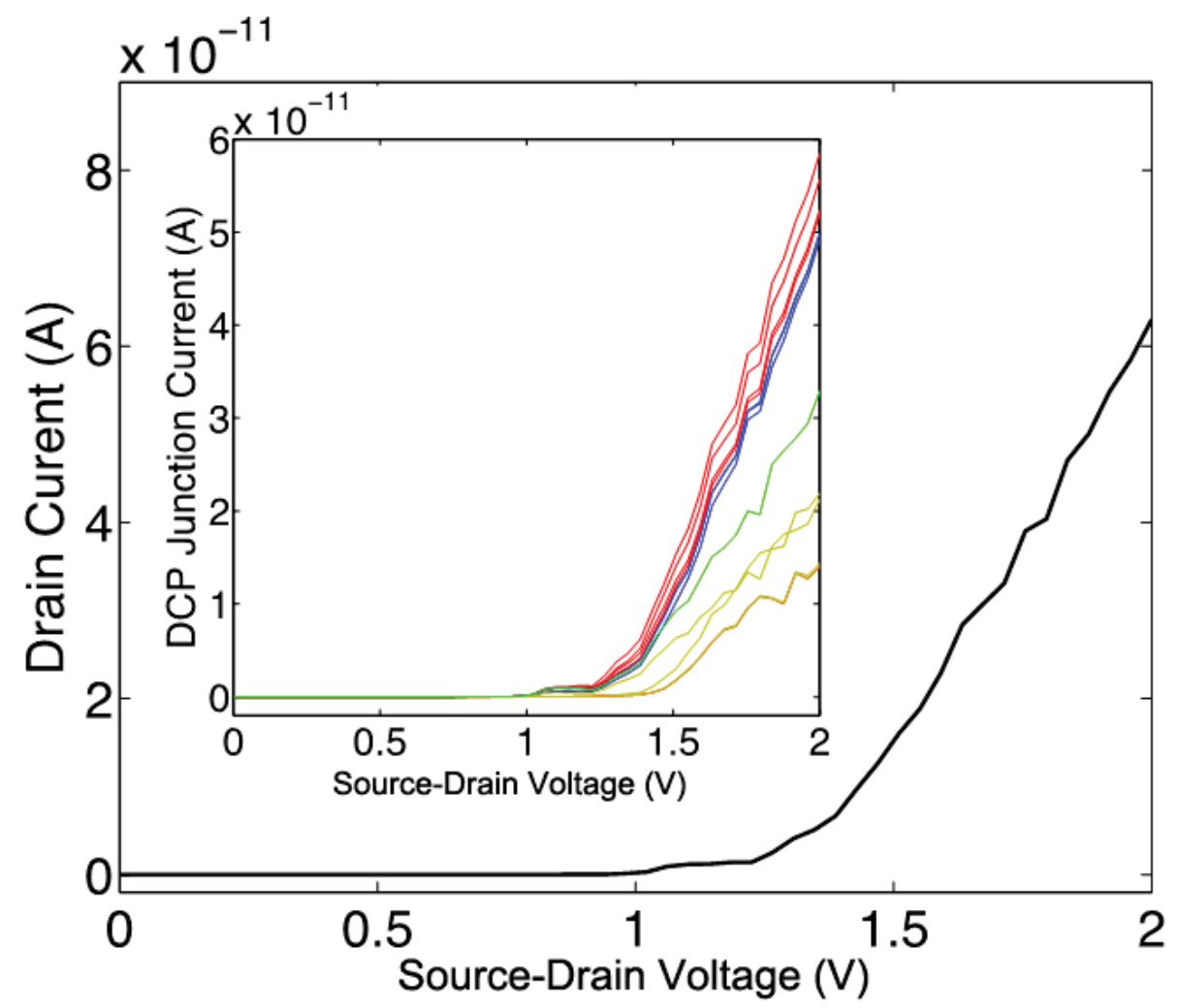

Figure 2.7: $I-V$ characteristics of a $2 \mathrm{D}$ device composed of 67 gold islands, as shown in figure 2.6 , at $\mathrm{T}=0 \mathrm{~K}$, and in the absence of a gate voltage. The inset shows the distribution of currents flowing through individual junctions in the dominant conducting path as a function of applied source-drain voltage bias.

as demonstrated in figure 2.8, which shows the variation in the junction resistances as a function of junction width, the wider junctions in a device experience larger voltage drops across them. Because the barrier heights depend on the voltage drops across the junctions, the wider junctions also therefore experience a larger decrease in their tunneling resistances as the source-drain bias is increased (from $12 \mathrm{~V}$ to $80 \mathrm{~V}$ ).

MITS simulations demonstrate power-law behavior of the $I$ - $V$ characteristics for $V_{s d}$ beyond the threshold voltage, consistent with experiments. The non-Ohmic behavior $(\zeta>1)$, in the simulations has been traced to the dependence of the barrier heights on the voltage drops across the junctions, which varies with charge state and with $V_{s d}$. 
Whereas Middleton and Wingreen [43] have argued that $\zeta$ should equal 1 and 5/3 for infinite 1D and 2D systems, respectively, in the limit of short screening lengths (weak capacitive coupling among islands), their computer simulations for finite systems gave $\zeta=1$ and $2.0 \pm 0.2$, respectively. A variety of experimental studies [40-42, 44, 45] give exponents ranging between 1 and 3 .

Our simulation studies show that the exponent $\zeta$ is sensitive to the disorder in the system and the length of the device [3]. The exponents also show a crossover from a lower value to a higher value as the source-drain bias is increased sufficiently. Such crossover behavior has also been observed in experimental devices [44].

Values of the threshold voltage $V_{t h}$ increase with increasing device length; however, prediction of $V_{t h}$ for a device with random island sizes and separations is an open question. Although there is no steady state current for $V_{s d}<V_{t h}$, as the applied voltage bias is increased across a device, but below the threshold, the charge state of the system changes in a discrete series of "up-steps" [40, 43]. Based on our MITS simulations, these changes in charge state can include the following, alone or in combinations: (i) a change in total charge on the device, (ii) advancement of the charge front across the device, or (iii) rearrangement of charge among the islands. Such transitions occur when $V_{s d}$ is increased sufficiently to bring some $\Delta W_{i, j}$ to zero making a transition energetically favorable. For example, in a simulation of a 25-junction device, increases in $V_{s d}$ necessary to overcome a total of 27 consecutive up-steps, as $V_{s d}$ is increased from zero to $V_{t h}$, range from $0.01 \mathrm{mV}$ to $153.7 \mathrm{mV}$. Once a transition takes place, others may follow until once again the system reaches equilibrium. At sufficiently high bias, the last up-step may be overcome, and the ensuing transition will take place with some rate determined by Eq. 2.1. This transition is a rate-determining step, as a subsequent cascade of transitions then take place quickly, leading to the advancement of one net electron across the device, but ultimately leading to the system coming back to its rate-determining step. Unfortunately, prediction 


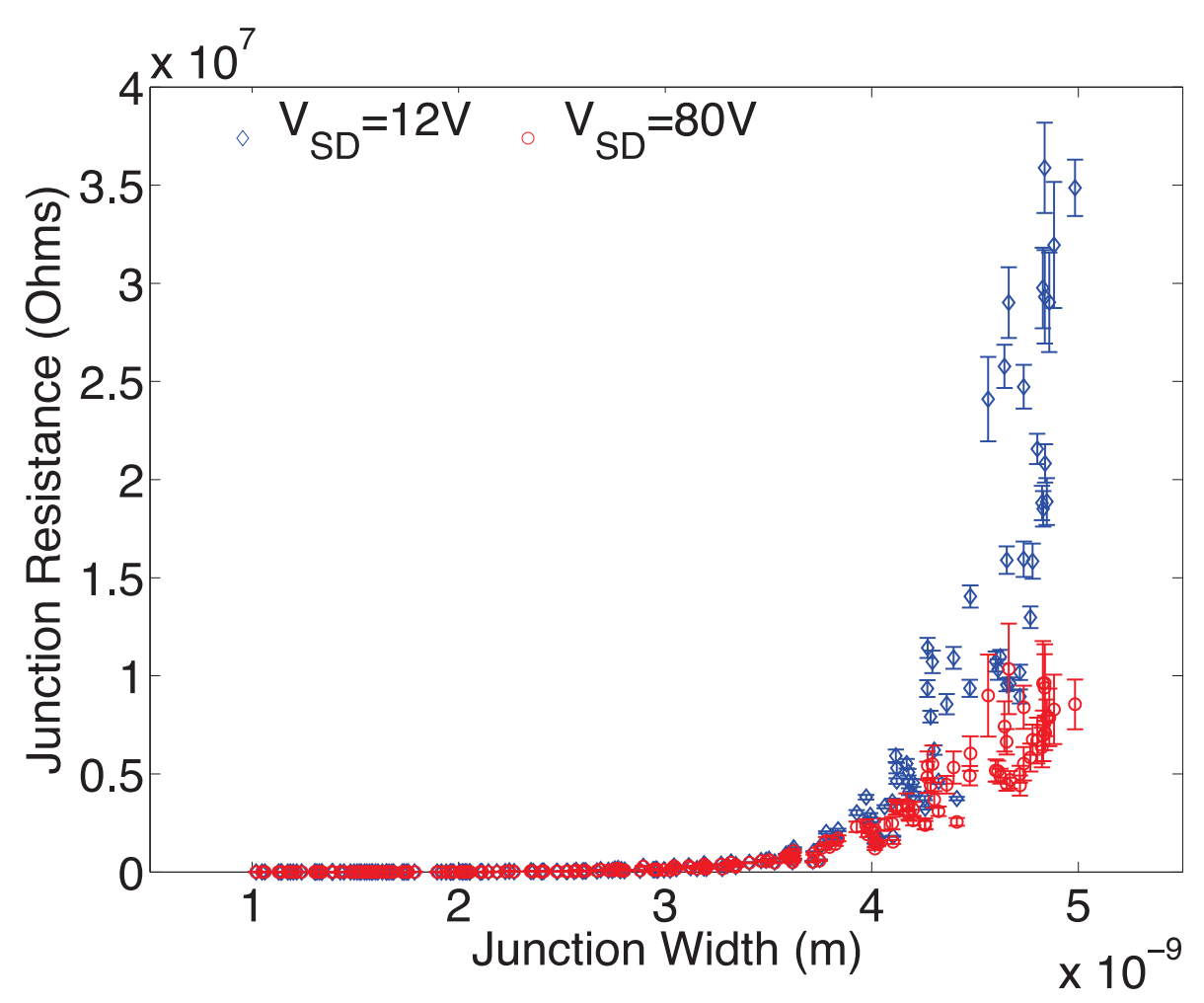

Figure 2.8: Junction resistances as a function of junction width, for a 200junction device, for two different source-drain biases, $12 \mathrm{~V}$ (blue diamonds) and $80 \mathrm{~V}$ (red circles). Note from figure 2.4 that at $12 \mathrm{~V}$ the device is in the Coulomb-staircase regime, while at $80 \mathrm{~V}$ it is in the power-law regime. Error bars represent standard deviations in the junction resistances averaged over 5000 Monte Carlo steps ( $\sim 1$ ns at $12 \mathrm{~V}$, and 18 ps at $80 \mathrm{~V}$ ) after reaching steady state currents. Reprinted with permission from Savaikar et al. [3]. Copyright 2013, AIP Publishing LLC. See appendix D for documentation of permission to republish this material.

of the individual up-steps and $V_{t h}$, based on a physical model of a $1 \mathrm{D}$ random device (materials, island radii, junction separations), appears to be impossible due to the capacitive junction couplings and dependence of the junction voltage drops on the charge state of the system and the applied bias.

With increasing $V_{s d}$ beyond $V_{t h}$, the currents change only slowly due to the slight changes in the junction voltage drops, that is, until some particular $V_{i, j}$ reaches a value such that its associated $\Delta W_{i, j}$ reaches zero and the charge state of the system 
changes. This can lead to the creation of a new conduction channel (sequence of allowed transitions in charge-state- $V_{s d}$ space that results in a net transfer of charge across the device), leading to a sharp increase in the current and the formation of a step in the CS. With increasing $V_{s d}$, ever more conduction channels open up, until at sufficiently high $V_{s d}$ the individual CS steps become indistinguishable.

With increasing temperature, key $\Delta W_{i, j}$ activation barriers for changes in charge state can be thermally overcome that lead to changes in charge state and to non-zero transition rates, even for $V_{s d}<V_{t h}$. Thus, thermal effects can lead to a non-linear decrease of the apparent threshold voltage of a device with increasing temperature, and rounding or elimination of steps in the CS structure. New CS steps can even manifest themselves $V_{s d}<V_{t h}$ due to the system attaining charge states at non-zero temperatures that are inaccessible at $\mathrm{T}=0$ and $V_{s d}<V_{t h}$. Further details of thermal effects in random 1D devices will be the subject of a future publication.

In experimental work on a 2D system of Au grains, by Cordan et al. [27] postulated the existence of a quasi-1D dominant conducting path (DCP) that carried most of the current across devices with a wide range of tunneling resistances. Our preliminary work using MITS has demonstrated the existence of a DCP in a random 2D device. The DCP shows robustness with changes in source-drain voltage and with moderate increases in temperature. The Coulomb staircase structure in the $2 \mathrm{D}$ device was less pronounced than in comparable 1D systems, however. This is likely due to the DCP being relatively optimized, and thus carrying a narrower range of tunneling resistances along the DCP than which exists among neighboring junctions in the overall 2D device. Our preliminary 2D simulations were also carried out on a relatively small system, leading to a short path length for the DCP. As shown above, the Coulomb staircase structure becomes less prominent as the device length decreases due to shorter devices having a lower probability of sampling unusually large junction widths. 


\subsection{Conclusions}

The MITS simulation package has proven to be a useful tool for modeling $I$ - $V$ characteristics of 1D and 2D arrays of nano-scale metallic islands under low and high biases, and gaining understanding of underlying mechanisms to explain the Coulomb blockade, Coulomb staircase, and power-law scaling behavior of the devices. The turn-on threshold source-drain bias depends strongly on the capacitances of the system, but because of the inherent randomness in island spacing and radii, the prediction of the threshold voltage based on the physical layout of a random device is not possible without carrying out the full simulation. With increasing source-drain bias the threshold is reached through a series of up-steps in which the charge state of the system changes, and the charge front eventually advances across the device. In agreement with the hypothesis of Cordan et al. [27], 2D systems with sufficient disorder have a robust dominant conducting path that carries most of the current across the device.

Future studies are planned to elucidate the effects of temperature and degree of ran-

domness on the behavior of such systems. Such insights may be helpful in using MITS to explore device designs with the goal of engineering desired device characteristics.

\subsection{Acknowledgments}

Simulation studies in this chapter were performed in part using the computing cluster wigner.research.mtu.edu in Information Technology Services and rama.phy.mtu.edu in the Department of Physics at Michigan Technological University. Y. K. Yap acknowledges the support from the U.S. Department of Energy, the Office of Basic Energy Sciences (Grant DE-FG02-06ER46294, PI:Y.K.Y.), the Center for Nanophase Materials 
Sciences at Oak Ridge National Laboratory (CNMS at ORNL) (Projects CNMS2009213 and CNMS2012-083, PI:Y.K.Y.), and by ORNL's Shared Research Equipment (ShaRE) User Program (JCI), which are sponsored by the Scientific User Facilities Division, Office of Basic Energy Sciences, U.S. Department of Energy (DOE). 


\section{Chapter 3}

\section{Calculation of Capacitance in Dense Disordered Systems}

\subsection{Introduction and Motivation}

Making an accurate calculation of the change in energy as charges move in a system is critically dependent upon having accurate knowledge of the capacitances in the system. Crude approximations to the capacitances can be calculated using the known analytical solutions such as the capacitance of an isolated sphere, the parallel plate capacitor, the isolated two-sphere system, etc. However the systems of interest in this study are quite dense, making these approximations differ markedly from the results of more sophisticated and accurate calculations. The finite element method (FEM) allows us to create systems with complicated geometries and find the electrostatic potential, electric field, surface charge density, and finally the capacitances of the system. It one of several state of the art methods for calculating capacitances in systems with complex geometry. The power of the FEM is great, but its utility is 
ultimately limited by the significant human and computational resources required to define a model system and run the calculations. To mitigate these hurdles, we have created tools that automate the setup of FEM calculations using open-source codes and allow for execution on computing clusters.

\subsection{Survey of the State of the Art}

The finite element method is one of several state of the art methods for calculating the capacitances in structures with complicated geometry. The primary competing methods are various boundary element methods [46-50], finite difference methods [51], and the floating random walk method [52-55].

Each of these approaches model a system of ideal conductors subject to an applied potential difference. They solve this boundary value problem in order to calculate the distribution of charge on the conductors when they are held at a fixed potential. From this the capacitance is easily calculated using the relationship $Q=C V$.

With the FEM, the region in which the electric field will be calculated is discretized using an unstructured 3D volume mesh. This volume is bounded by the conductors of interest, which themselves are discretized using an unstructured 2D surface mesh. The potential $\phi(\vec{x})$ throughout the volume is described by Laplace's equation,

$$
\nabla^{2} \phi(\vec{x})=0
$$

recast in the integral or weak form,

$$
\int_{\Omega}\left(\nabla^{2} \phi(\vec{x})\right) w(\vec{x}) d \vec{x}=0
$$


where $w(\vec{x})$ can be any arbitrary function. The electrostatic potential within each element is approximated with piecewise continuous polynomials. A trial function representing the solution throughout the whole volume is then the sum of these piecewise elements. The potential at the boundaries is defined, and the variational principle is used to find a global solution that minimizes the residual, where the residual is the integral throughout the volume of the Laplace operator acting on the trial function.

The finite volume method is closely related to the finite element method. The Laplace equation is also expressed in a weak or integral form, however Gauss's law is used to transform the volume integral into a surface integral. The problem is then represented in terms of fluxes through the surfaces of the elements.

In the finite difference (FD) method, the volume is typically discretized using a structured mesh. The Laplace equation throughout the volume is represented in the differential or strong form, and the solution is found using an iterative method.

Boundary element methods ignore the volume and calculate directly what surface charge densities would be consistent with the desired Dirichlet boundary conditions. This is done by discretizing the surfaces into boundary elements and setting up a system of equations describing the potential at each element in terms of sums of integrals over the surface charge densities of the other elements. In the simplest case, the potential for each element is evaluated only at the center of the element, and the surface charge is represented by a point charge at the center of the element. In this formulation the potential at each element is proportional to a sum over all other elements of the charge divided by the distance between the element and that charge. This describes a dense matrix equation that can be solved for the unknown surface charges. The fast multipole method (FMM) can reduce the computational cost of the BEM by grouping charges together and approximating the electrostatic potential distant from the group of charges with a multipole expansion [46]. 
The floating random walk (FRW) method [52] makes use of Monte Carlo methods and the mean-value-theorem to calculate the electrostatic potential on a Gaussian surface surrounding an individual conductor. It is a fast and efficient method that is well established in the semiconductor industry for calculating the 'parasitic' capacitances associated with the wiring in very large scale (VLSI) integrated circuits.

Not all of these methods would have been possible to use for our purposes. For example, it appears all FRW method codes are designed to exploit the rectilinear nature typical of VLSI structures, and it does not appear to be possible to extend the method to arbitrary geometries while maintaining an acceptable level of performance. We chose to use the FEM for several reasons. It is a method that can handle arbitrary geometry, and for which there is a rich ecosystem of open source software implementing the method. We chose the SALOME [22] open-source computer aided engineering (CAE) package to set up the geometry and create the surface and volume meshes. We chose the Elmer finite element package [23] for setting up and solving the FEM equations, and calculating the capacitance matrix from the results. This allowed us to programmatically define the geometry and tune the meshing parameters, allowing for good integration with our kinetic Monte Carlo simulation workflow.

\subsection{Computational Methodology}

\subsubsection{Capacitance Matrix}

There are multiple possible approaches to calculating capacitances within the finite element method (FEM) approach. In each case the underlying physics is the same. We begin by relating the charge $Q$ accumulated on a conductor to the electrostatic 
potential difference $V$, via the capacitance $C$ :

$$
Q=C V
$$

For a simple two-conductor system, the charge on each conductor is equal and opposite and the relevant potential $V$ is the difference in potential between the two conductors. For a system of $N$ conductors, we can write a matrix equation:

$$
\vec{Q}=\mathbf{C} \vec{V}
$$

where $\vec{Q}$ is a vector containing the net charge on each conductor, $\vec{V}$ is a vector containing the potential of each conductor with respect to a common reference, and $\mathbf{C}$ is the Maxwell capacitance matrix, a $N \times N$ matrix derived from the mutual capacitance between each conductor [56]. The Maxwell capacitance matrix is constructed from the mutual capacitances $c_{i, j}$ between the $i^{\text {th }}$ and $j^{\text {th }}$ electrodes. In this terminology the self capacitance of an object is the mutual capacitance between the object and ground, and the total capacitance is the sum of all the mutual capacitances associated with the object. The Maxwell capacitance matrix is defined as

$$
C_{i, j}= \begin{cases}\Sigma_{j \neq i} c_{i, j} & \text { if } i=j \\ -c_{i, j} & \text { otherwise }\end{cases}
$$

where the diagonal elements are the total capacitances and the off-diagonal elements are the mutual capacitances multiplied by -1 . Since $c_{i, j}=c_{j, i}$, this matrix is symmetric and can be determined from the $N(N-1) / 2$ unique $c_{i, j}$ elements.

With the FEM method, we impose constant-potential boundary conditions on each of the conductors, and solve for the surface charges on each conductor. By judicious choice of potentials $(\vec{V})$, enough information is obtained from one calculation to determine one column of the capacitance matrix $\mathbf{C}$. Thus, with $N$ FEM calculations 
we can determine the $N(N-1) / 2$ unique elements of $\mathbf{C}$.

Specifically, the potential is set to zero for all except the $i^{\text {th }}$ conductor, which is set to unity. Pre-multiplying this voltage vector $\vec{V}$ by the capacitance matrix returns the $i^{\text {th }}$ column of the capacitance matrix. Meanwhile, the left-hand-side of the equation is an array containing the charge on each island, as calculated by the FEM method with the potentials set as previously mentioned.

\subsubsection{Finite Element Calculation}

We use the finite element method (FEM) to calculate the capacitances in a system of arbitrarily shaped conductors. However, the FEM is not able to do this directly. There are several steps involved, including creating a geometrical representation of the system, creating a mesh from the geometry, calculating the electrostatic potential in the vacuum, calculating the electric field in the vacuum, calculating surface charges, integrating to find surface charges, and finally relating the surface charges to the capacitances.

\subsubsection{Geometry}

The first step is to create the geometrical representation of the system. For this we use the open-source computer aided engineering (CAE) package SALOME [22]. SALOME exposes a Python-based text user interface, which allows for the operation

of the program to be directed by a Python program. A set of Python and Bash scripts were written to read in a file describing the geometry of the system, and to create the geometrical model within SALOME. 


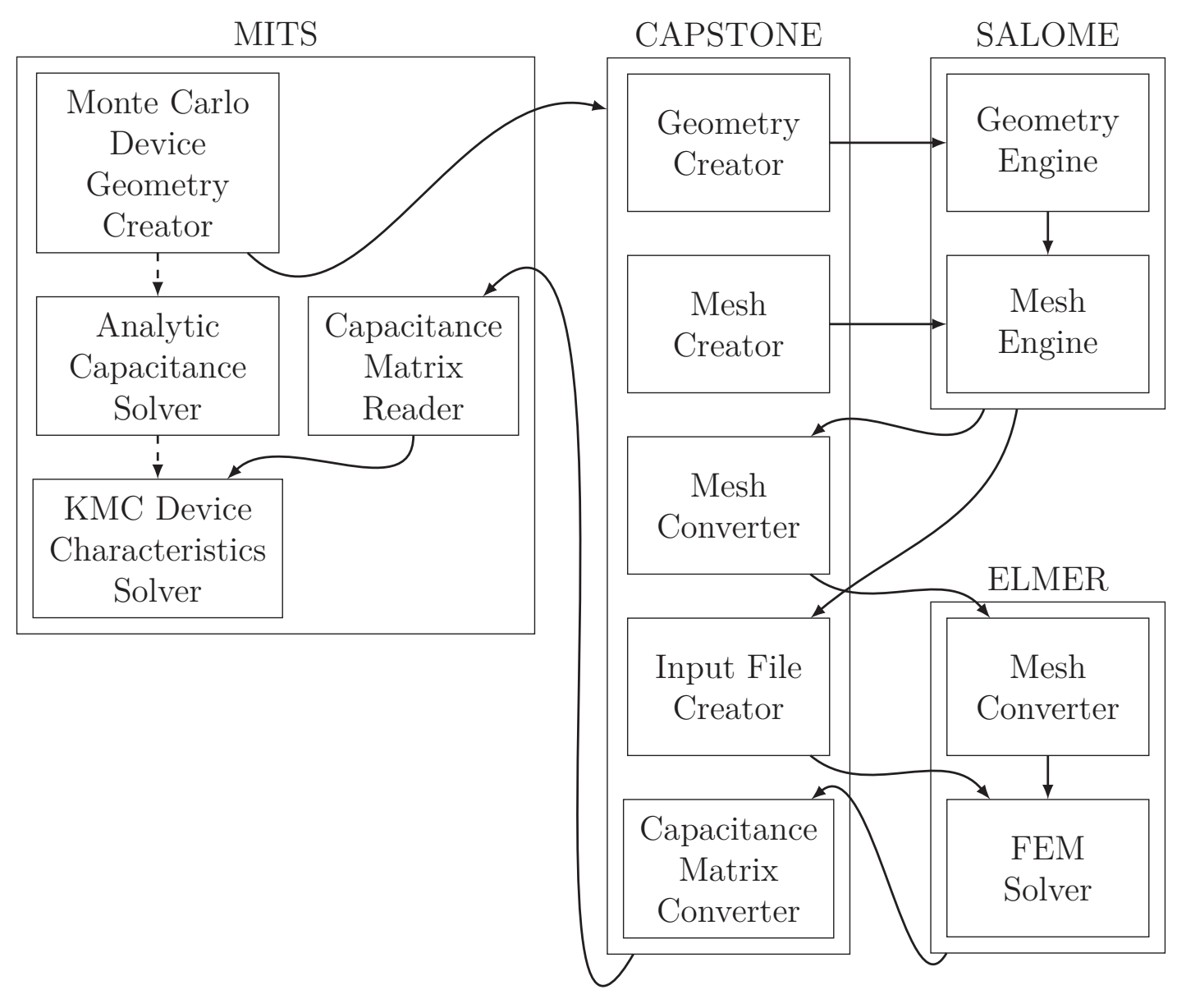

Figure 3.1: A 2D MITS simulation begins with creating a randomized device using a hard-sphere Monte Carlo algorithm. This device geometry is then fed to an analytic capacitance solver which generates the capacitance matrix. This capacitance matrix is an input to the kinetic Monte Carlo device simulator, which calculates current-voltage characteristics. With our FEM capacitance calculations, the analytic capacitance solver in MITS is bypassed and the device geometry is sent to our CAPSTONE capacitance solver package. CAPSTONE interfaces directly with the SALOME package and directs SALOME to build a 3D model of the system and then to discretize the model into a FEM mesh. Elmer is used to convert the mesh, and CAPSTONE creates an Elmer input file based on the output of the SALOME meshing routine. The FEM calculation is run, and output is the mutual capacitance between each pair of conductors. MITS is then used to convert this matrix of mutual capacitances into a Maxwell capacitance matrix, and finally to simulate the characteristics of the device. 


\subsubsection{Mesh}

Next the volume and surfaces described by the geometry need to be discretized, creating a FEM mesh. Again we use SALOME, directed via a Python program.

The volume of interest is not the volume of the conductors, but rather the vacuum surrounding the conductors. We are interested in simulating an isolated system, but this requires an infinitely large volume. Therefore we approximate the isolated system by enclosing the system in a "large enough" computational box. In order to choose how large of a bounding box is large enough, we ran calculations using progressively larger boxes and observed that all relevant results converge towards limiting values. We chose a size that was estimated to match the isolated result within several percent.

The surfaces are meshed by first discretizing the one-dimensional elements of the surfaces, and then creating the two-dimensional mesh beginning from the 1D mesh. For the bounding box, this is straightforward, the edges of the box are split into equal segments, and a triangular mesh is create based on the linear 1D mesh. However the islands are modeled as spheres, and so by default the 1D elements are chosen to be a half-circumference, beginning and ending at the north and south poles. This causes trouble for the $2 \mathrm{D}$ meshing algorithm, due to the singularity at the poles. We get around this by splitting the sphere into two surfaces divided at the equator. This allows us to use a linear 1D mesh and avoid the instability of the meshing algorithm at the poles.

Once the surfaces are meshed, the volume of the vacuum is filled with a tetrahedral mesh. The 2D elements are grouped according to the conductors they represent. The resulting mesh is exported in the I-Deas Universal (UNV) format. See figure 3.3 for an example of a two-dimensional surface mesh, and figure 3.5 for an example of the 
three-dimensional volume mesh.

\subsubsection{Finite Element Method Calculation}

The open source Elmer finite element package [23] is used for the actual FEM calculation. The set of Bash and Python programs that orchestrate the creation of the mesh with SALOME also orchestrate running Elmer and post-processing the results. This begins with converting the mesh from the UNV format to Elmer's native format. Next the input file is created based on a standard template and extended to include a description of each electrode.

The capacitances are calculated in a multi-step process. First, one single electrode (surface) is set 'high,' meaning the voltage on that electrode is set to $1 \mathrm{~V}$. All other electrodes are grounded, i.e. the voltage is set to zero. With the electrostatic potential $\phi$ defined on all the bounding surfaces, Laplace's equation,

$$
\nabla^{2} \phi=0
$$

is solved in the vacuum, delivering the electrostatic potential throughout the volume. From the electrostatic potential, the electric field is calculated:

$$
\vec{E}=-\vec{\nabla} \phi
$$

The surface charge density is calculated from the discontinuity of the perpendicular component of the electric field at the surface of the conductor:

$$
\sigma=-\varepsilon_{0} \Delta \overrightarrow{E_{\perp}}
$$


The electrodes are assumed to be perfect conductors. Therefore there is no tangential component to the electric field, and the electric field inside the conductors is zero. Thus the discontinuity of the electric field at the surface is simply equal to the magnitude of the electric field at the surface. Additionally, for a conductor in equilibrium, all the charge is on the surface. Finally we can calculate the charge $Q_{i}$ on the conductor $i$ by integrating the surface charge density over the surface of the conductor, $\Omega_{i}$.

$$
Q_{i}=\int_{\Omega_{i}} \sigma d A=\varepsilon_{0} \int_{\Omega_{i}}|\vec{E}| d A
$$

As outlined above, a single FEM calculation tells us the charges $Q_{i}$ that must be placed on each of the $N$ conductors in order to generate a potential $\phi_{j}$ on each of the conductors. By systematically changing the potentials and calculating the charges, we can calculate, column-by-column, the capacitance matrix $\mathbf{C}$.

\subsection{Test Systems}

The capacitance calculation capabilities were tested with a series of progressively more complicated (and interesting) test cases.

\subsubsection{Sphere Within a Sphere}

We begin with a model that has a simple analytic solution: the isolated sphere. The capacitance of an isolated sphere of radius $r$ is

$$
C_{\text {Sphere }}=4 \pi \varepsilon_{0} r
$$


However, as mentioned above, we cannot represent a truly isolated system. Therefore a more useful model is the sphere within a larger sphere, which has a mutual capacitance of

$$
C_{\text {Sphere-Sphere }}=\frac{4 \pi \varepsilon_{0}}{\frac{1}{r}-\frac{1}{R}} .
$$

This is a model that we can represent with arbitrary precision, limited only by the fineness of the mesh we are willing to create.

We used two test cases: a smaller system were the ratio of outer radius to inner radius is 10 , and a larger system where the ratio is 100 . In figure 3.2 , we see that a simple mesh with a small number of elements is able to generate a result within $3 \%$ of the exact value for both system. Interestingly for the smaller system, a moderate increase in the fineness of the mesh is able to reduce the error to $1 \%$, while for the larger system, the error doesn't reduce down to that level even for a rather fine mesh. This is likely due to the method we used for increasing the fineness of the mesh, which increased the mesh density uniformly instead of targeting the regions where the errors were occurring. In later work we were more careful in our refining of the mesh.

\subsubsection{Single Impinging Sphere}

In the next test system, we again modeled an isolated sphere by enclosing it in a much larger sphere (with radius 100x the radius of the smaller sphere). Then we brought another similar sphere nearby the first and calculated the impact the additional sphere has on the self capacitance of the first sphere. The self capacitance of the central sphere is defined as the mutual capacitance between the central sphere and the bounding sphere. In figure 3.3, the first sphere is held a fixed potential of +1 V relative to ground, while the impinging sphere and the outer sphere are grounded. Shown in the figure are isopotential surfaces, colored according to potential, with blue 


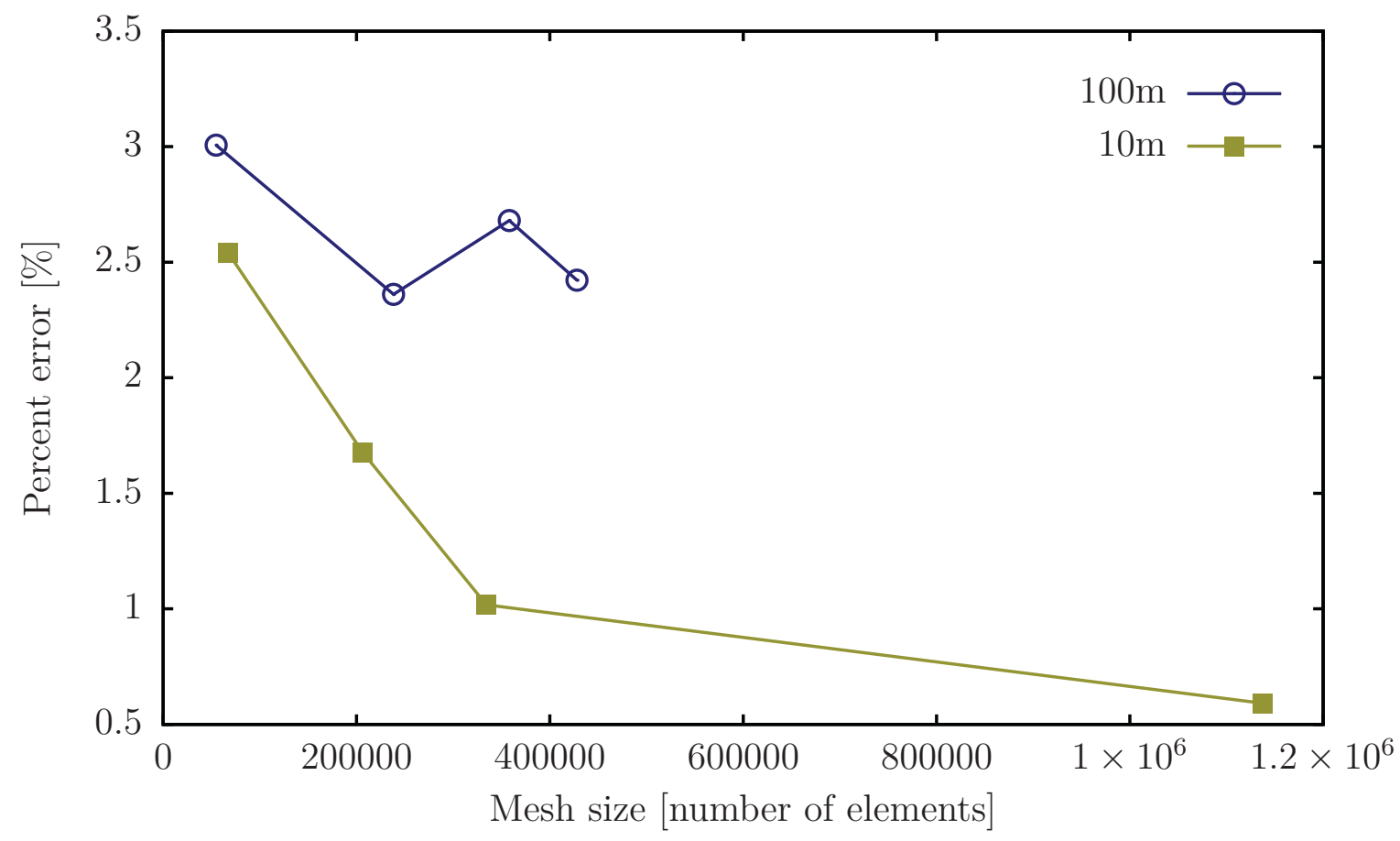

Figure 3.2: The finite element method is used to calculate the capacitance of a spherical capacitor with ratio of outer radius to inner radius of 100 (purple) and 10 (green). Percent difference between the calculated result and analytic result is on the vertical axis, while the horizontal axis is a measure of the quality of the mesh, approximated by the number of elements in the mesh.

equal to zero (ground) and red equal to +1 volt.

The self capacitance of the first sphere decreases as the second sphere is brought closer (figure 3.4). This can be envisioned as the first sphere being shielded from ground (represented by the larger enclosing sphere) by the presence of the second sphere. Interestingly, as the second sphere approaches, the self-capacitance appears to approach a limiting value of approximately $72 \%$ of the value for an isolated sphere. 

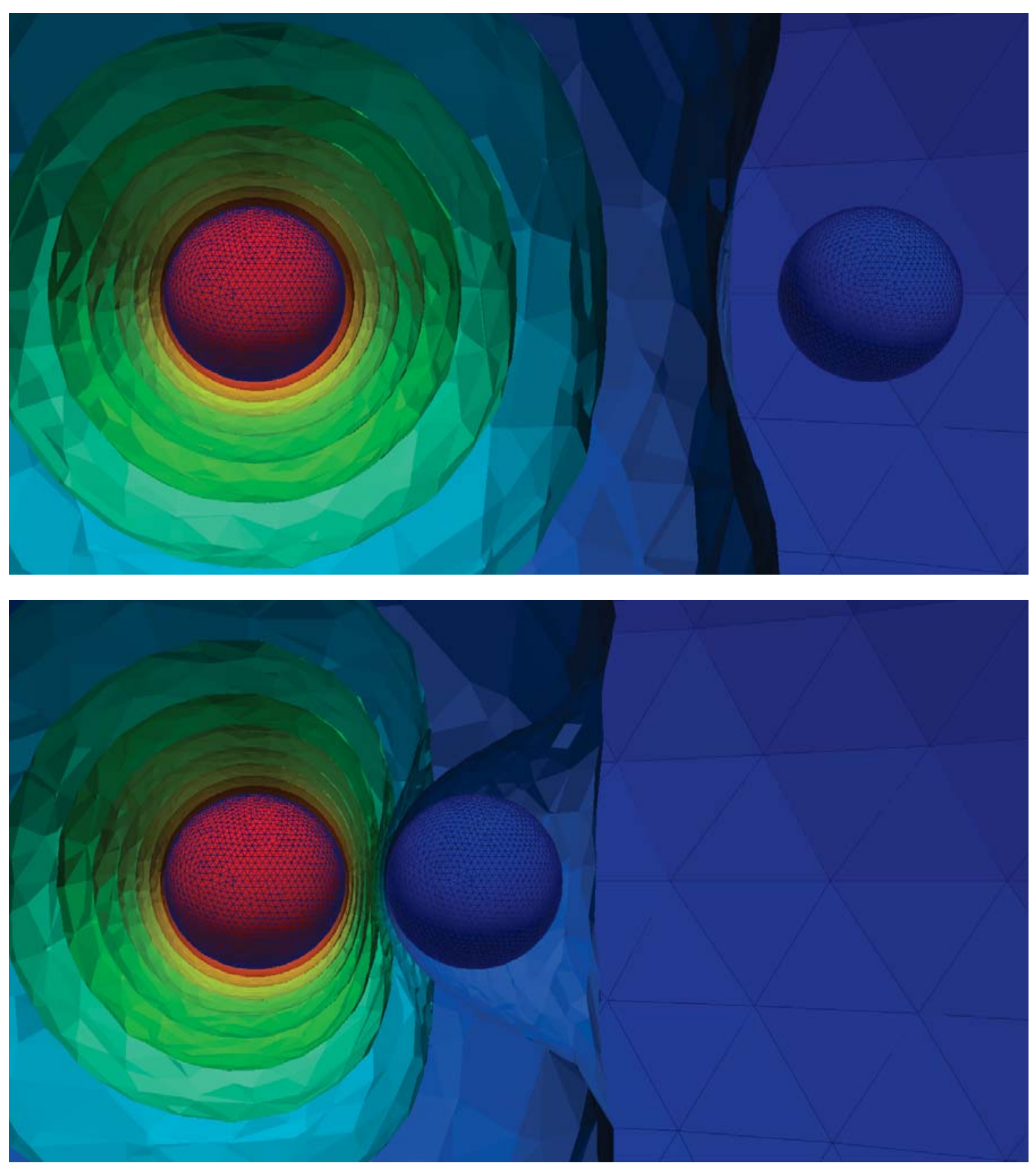

Figure 3.3: The finite element method is used to calculate the electrostatic potential in a system consisting of a sphere with an applied bias relative to ground, and with another grounded sphere impinging upon it. Both spheres have the same radius, and the distance between the two spheres is 5 radii in the first image, and 0.5 radii in the second image. Isopotential surfaces are calculated for every tenth of a Volt from zero (blue) to $1 \mathrm{~V}$ (red). 


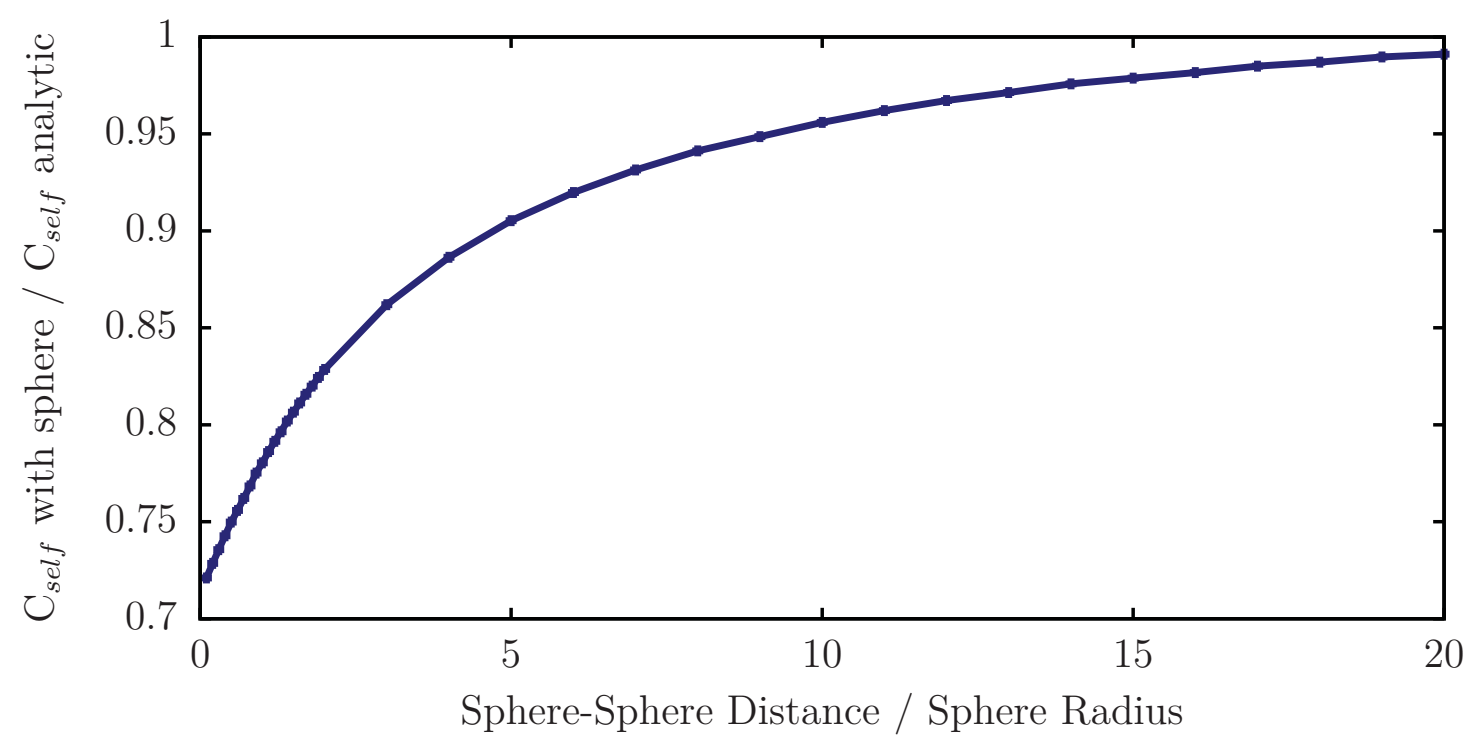

Figure 3.4: The finite element method is used to calculate the capacitance of an isolated sphere with another sphere impinging upon it. Both spheres have the same radius, and the distance between spheres is given relative to the radius (horizontal axis). The capacitance is shown normalized to the self capacitance of an isolated, identically sized sphere.

\subsubsection{Multiple Surrounding Spheres}

Next we investigated the effect of bringing multiple spheres near to the first sphere. We started with a single sphere within a sphere that is 100x larger, and added more equal sized spheres around it until there were seven spheres in a hexagonal close pack (HCP) arrangement. Figure 3.5 demonstrates this arrangement, showing a cutaway view of the mesh for the system with five spheres surrounding the first sphere.

The effect these additional spheres have on the self capacitance is shown in figure 3.6. The self capacitance and total capacitance of the central sphere is graphed as a function of the number of neighbors, and is normalized to the analytic value for the capacitance of the single sphere-within-a-sphere system. As expected, the self capacitance is diminished as more neighbors are added, further shielding the 


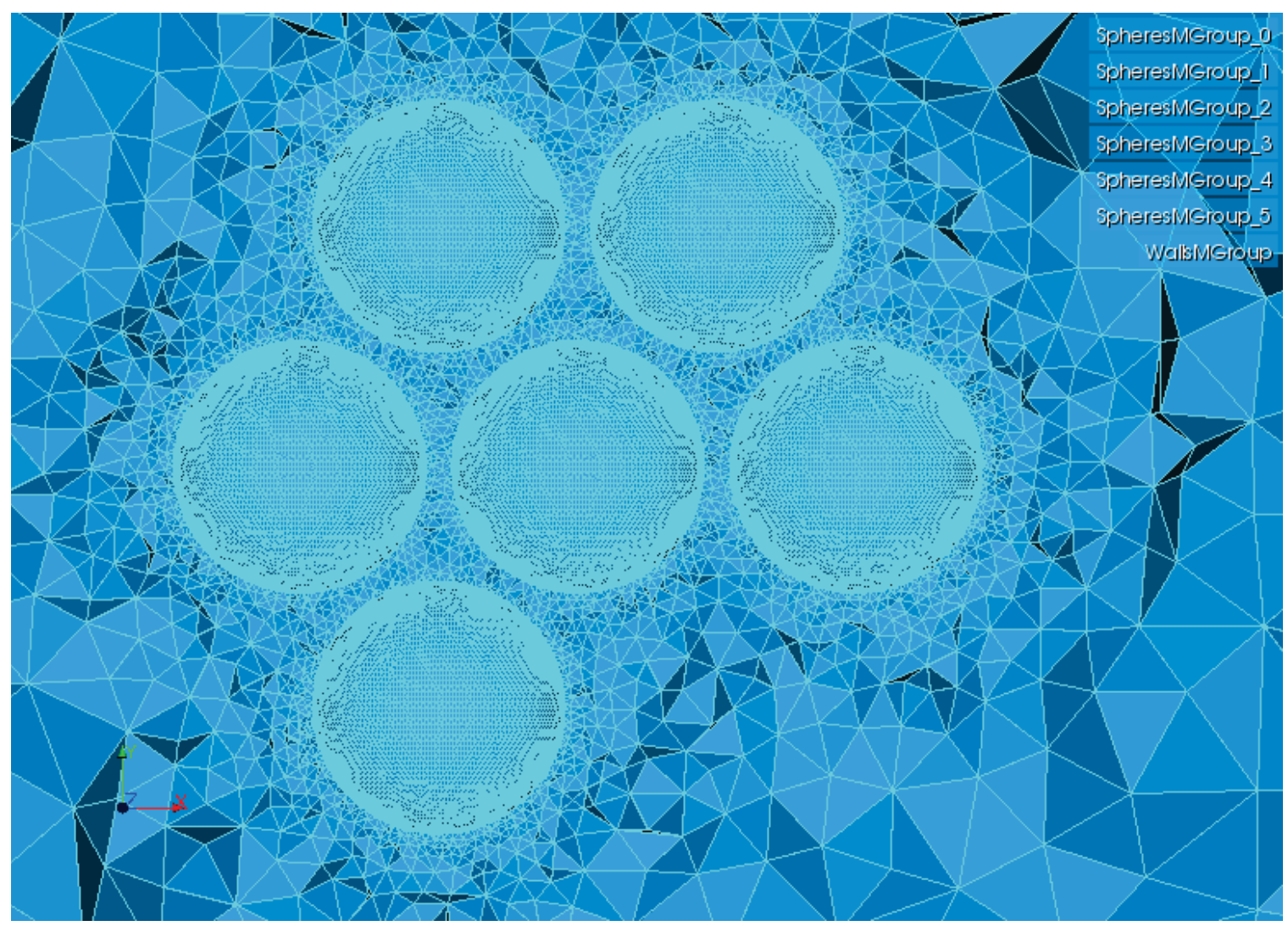

Figure 3.5: Cutaway view of a mesh for a system with five impinging spheres in a hexagonal close pack (HCP) arrangement.

central sphere. Additionally, the total capacitance increases, as the effective dielectric constant increases for the region between the central sphere and the bounding sphere.

Next the density of the neighboring spheres is modified by changing the distance of closest approach between the spheres. Figure 3.7 shows the self capacitance as a function of the distance between the six neighboring spheres. Two interesting things can be noted in this graph. First, the self capacitance of the central sphere is less than that of an isolated sphere, and decreases as the neighbors are brought closer. This can be anticipated as the neighbors act to shield the sphere from ground. Conversely, the total capacitance of the sphere is greater than for the isolated sphere, and increases as the neighbors come near. We can anticipate this result as well if we imagine the 


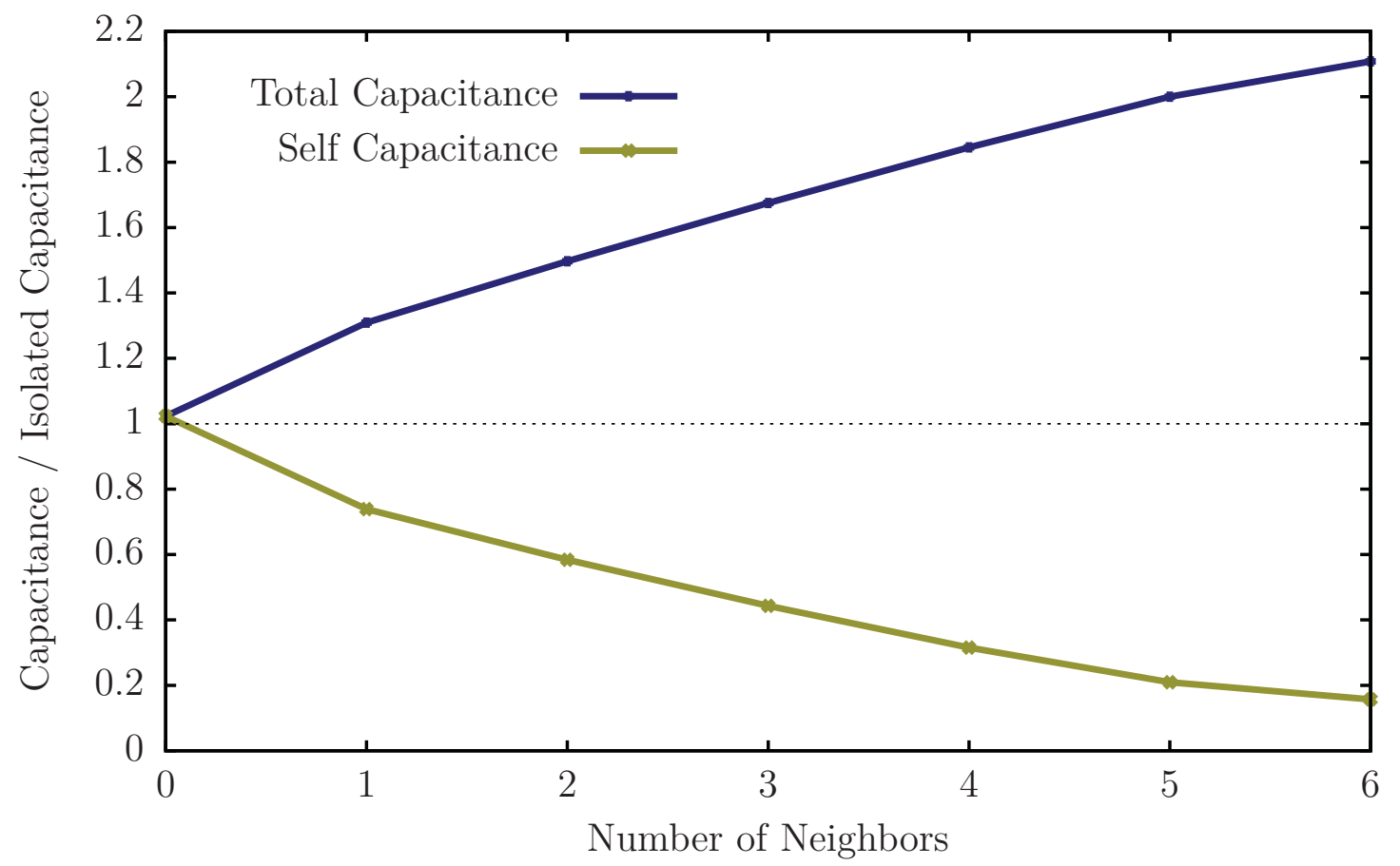

Figure 3.6: Self capacitance is calculated as a function of the number of neighboring spheres.

vacuum and surrounding spheres to be a material with an effective dielectric constant greater than unity.

\subsubsection{Potential Energy Barriers}

When we consider the problem of tunneling from one island to the next, we generally assume that the potential energy barrier separating the islands varies linearly from one island to the next. This is the trapezoidal barrier approximation [57]. In this study we calculated the potential energy barrier along a line of closest approach between the islands for a system consisting of two larger electrodes and three islands. Figure 3.8 shows the electrostatic potential on a plane that cuts through the center of the spheres. In this model, the islands are $1 \mathrm{~nm}$ in diameter and are separated from 

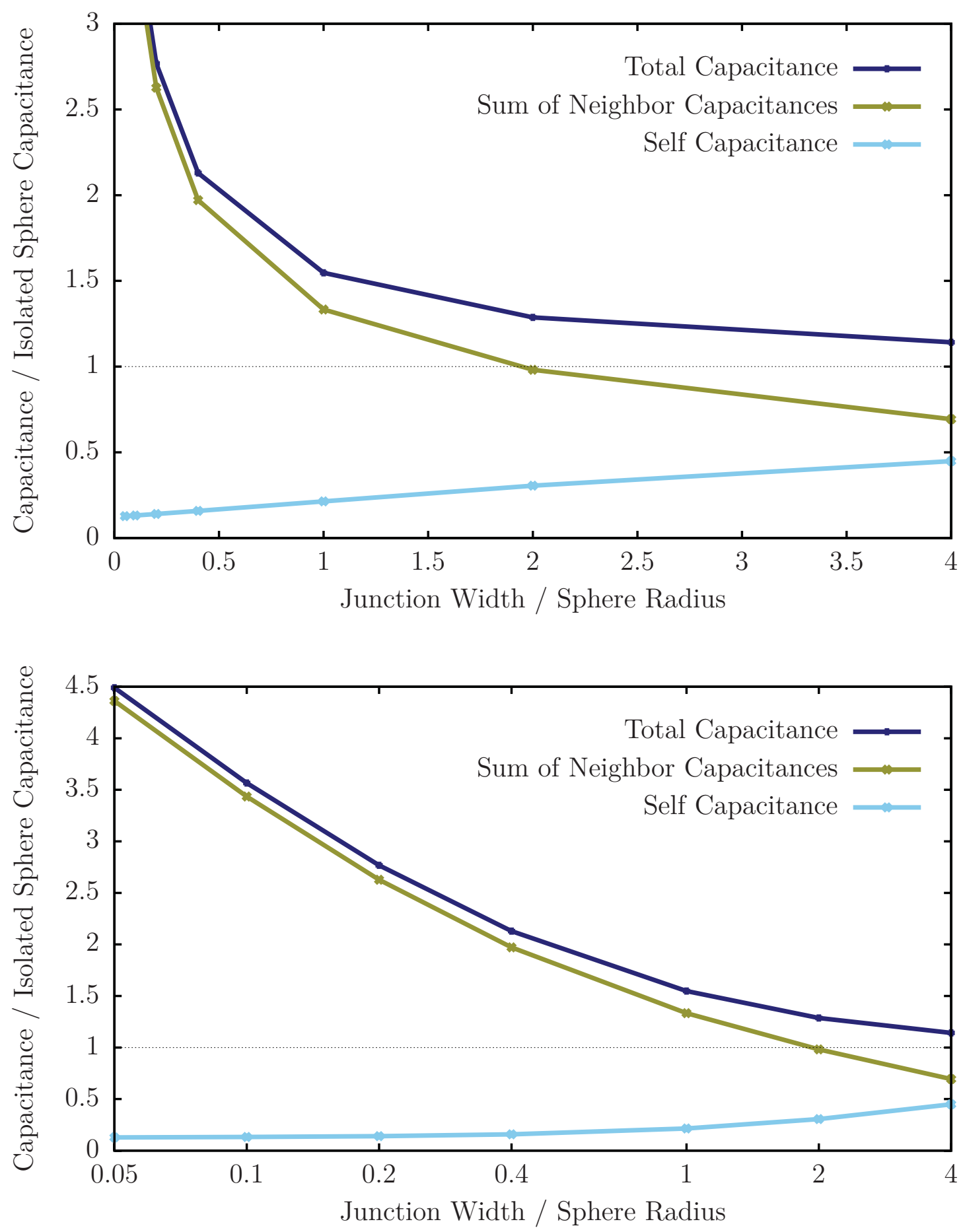

Figure 3.7: (a) Self capacitance is calculated as a function of the density of neighboring spheres, which is manipulated by changing the distance of closest approach between the spheres. (b) The same data is graphed on a semi-log scale. 


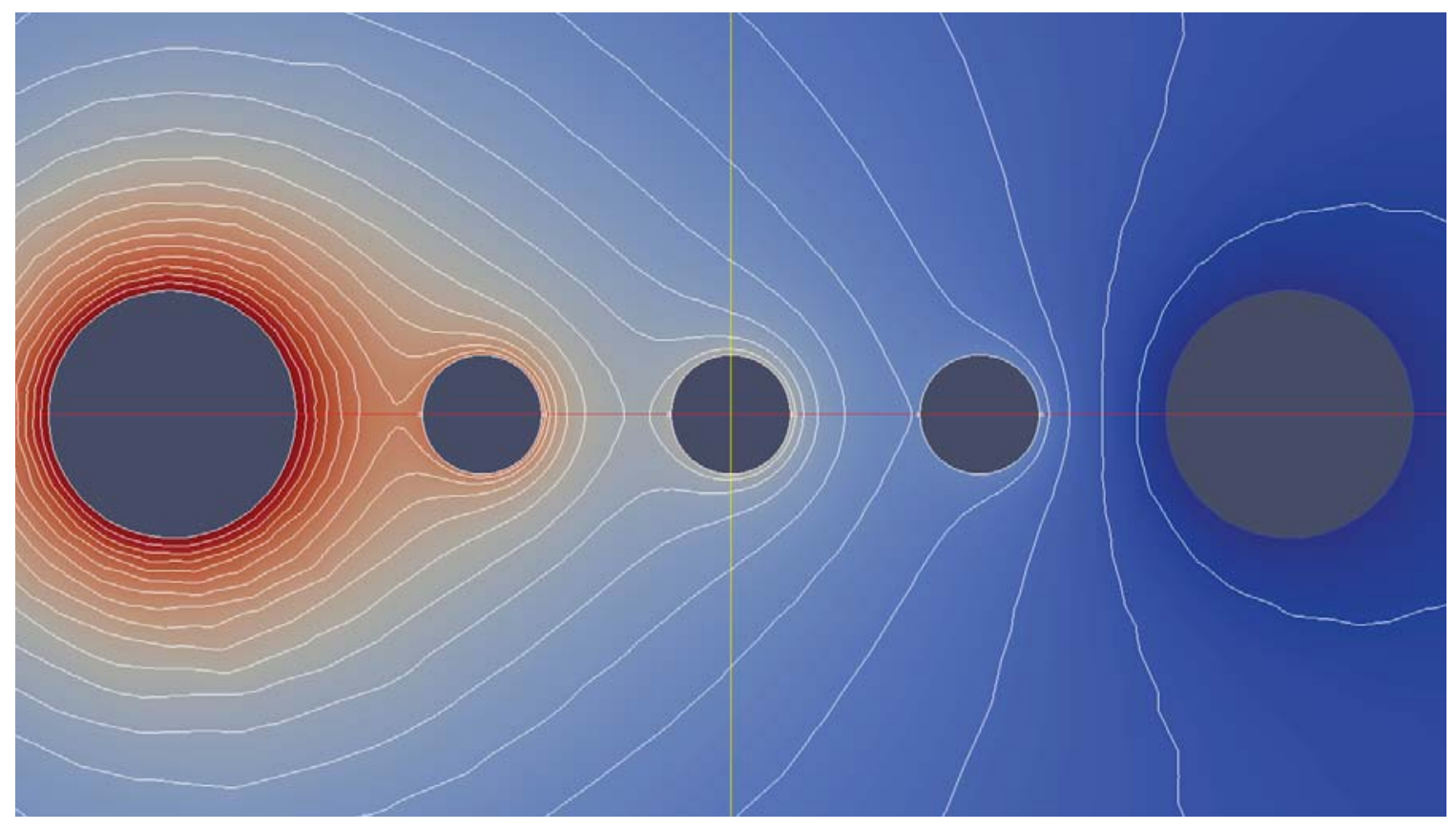

Figure 3.8: We simulated $1 \mathrm{~nm}$ diameter islands between $2 \mathrm{~nm}$ diameter electrodes, separated by $1 \mathrm{~nm}$ tunnel junctions. The leftmost electrode is at $-4 \mathrm{~V}$, and the next three islands are at $-3,-2$, and $-1 \mathrm{~V}$, respectively. The rightmost electrode and the bounding box are grounded. Isopotential lines are drawn every $0.2 \mathrm{~V}$.

one another and the electrodes by $1 \mathrm{~nm}$ junctions. The electrodes are spheres $2 \mathrm{~nm}$ in diameter. The right-most electrode and the bounding box are grounded, and the islands, from right to left, are at 1,2 , and $3 \mathrm{~V}$, with the left-most electrode at $4 \mathrm{~V}$.

In figure 3.9 we see that the barrier is modified enough that we would expect to see a measurable change in the effective resistance of barrier. In particular, the lowering of the first barrier would be expected to decrease the resistance of the junction by a significant amount. If instead the left electrode and islands were negatively biased relative to ground, we would see an increase in the barrier height and a corresponding increase in the effective resistance of the junction. The effects of this barrier modification are explored further in section 4.3.1. 


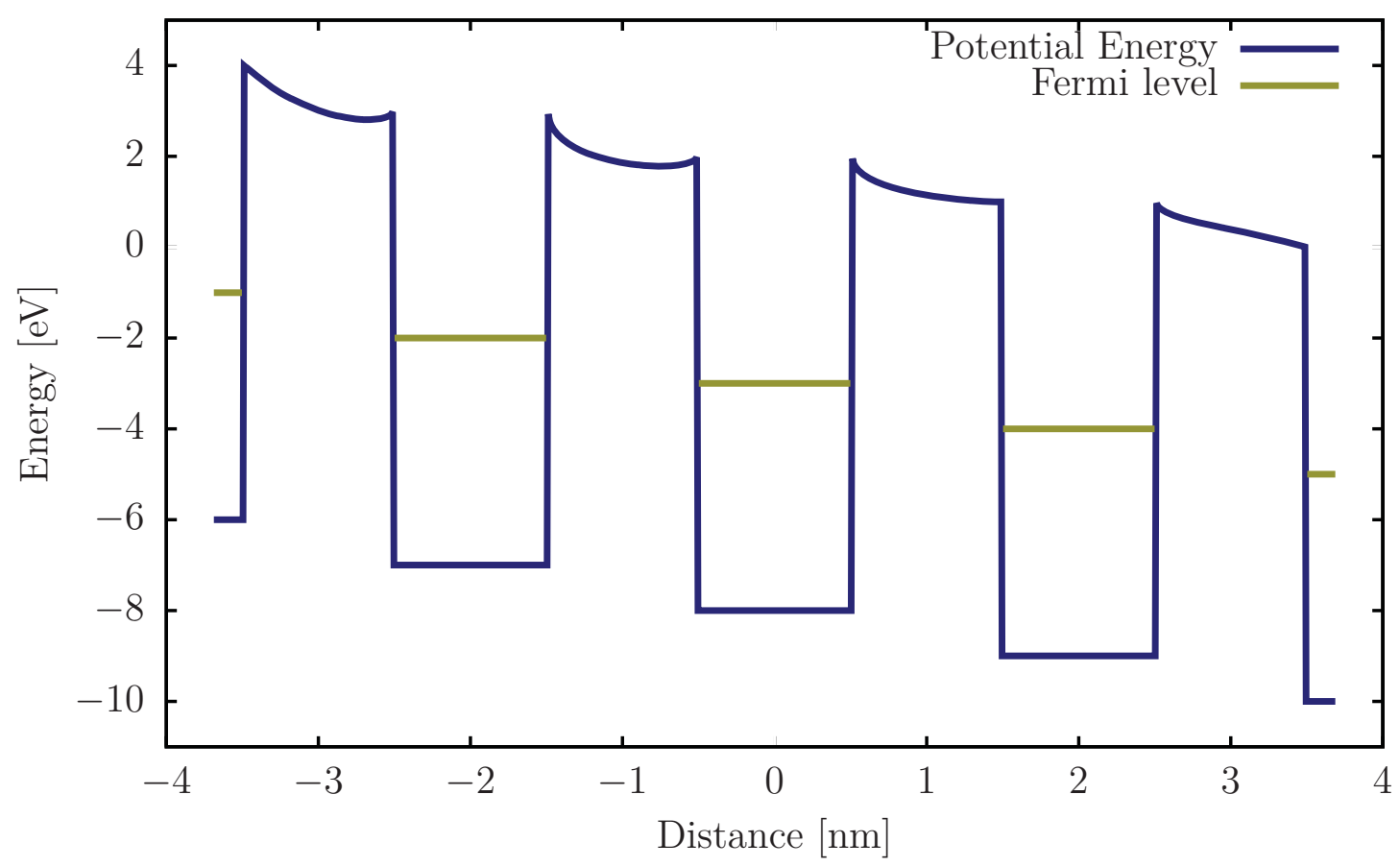

Figure 3.9: An energy diagram illustrating the modification of the barrier by the electric field between the islands. The potential well and Fermi level represent a system with a well depth of $5 \mathrm{eV}$ and work function of $5 \mathrm{eV}$. The shape of the barriers were calculated from the system described above with three $1 \mathrm{~nm}$ islands between two electrodes.

\subsection{Applications}

We applied the method described in this chapter to three types of systems, which are all variations on a theme of multiple-island Coulomb-blockade devices. The first is a linear Coulomb-blockade device described in more detail in chapter 2. For the second application, we bend the linear device and investigate the stability of the device properties as a function of curvature. The third is a planar Coulomb blockade device which allows conduction through a two-dimensional network of islands. Each of these applications are electronic devices that either allow or don't allow a current to flow depending on the tiny changes in electrostatic energy associated with the movement of 
single electrons. Because of the extraordinary sensitivity of these devices, an accurate description of the electrostatics of the system (through knowing the capacitances in the system) is essential to accurately modeling this class of device.

\subsubsection{Linear Chain}

We calculated the capacitances in a linear chain of spherical metallic islands to support efforts to model the electrical conduction properties of so-called QD-BNNT transistors. These quantum dot boron nitride nanotube (QD-BNNT) transistors are created by sputtering gold or iron onto insulating boron nitride nanotubes (BNNTs). This results in a quasi-one-dimensional array of metallic islands supported by an insulating substrate.

Previous modeling efforts were reported in publications by Lee et al. (Advanced Materials 25, 4544-4548 (2013)) [2] and Savaikar et al. (Journal of Applied Physics 114, 114504 (2013)) [3]. In that work we approximated the capacitances in the system based on analytic solutions for the capacitance of isolated spheres and pairs of spheres. For example, the self capacitance of each island, i.e. the mutual capacitance between the island and ground, is taken to be $4 \pi \varepsilon_{0}$ times the radius of the island. This is the exact result for an isolated sphere, but our systems are dense enough that the isolated approximation needs to be evaluated. The mutual capacitance between two spheres in isolation can be found using the image charge method [34]:

$$
c_{i, j}=-\frac{4 \pi \varepsilon_{0} r_{i} r_{j}}{d_{i, j}} \sinh (\alpha) \sum_{n=1}^{\infty}\left|r_{i} \sinh (n \alpha)+r_{j} \sinh ((n-1) \alpha)\right|^{-1}
$$

where $r_{i}$ and $r_{j}$ are the radii of the spheres, $d_{i, j}$ is the distance between the centers 
of the spheres, and $\alpha$ is defined for convenience as

$$
\alpha=\operatorname{arcCosh}\left(\frac{d_{i, j}^{2}-r_{i}^{2}-r_{j}^{2}}{2 r_{i} r_{j}}\right)
$$

Panel (a) in figure 3.10 shows the difference between our calculated self capacitances and the analytic approximation. The calculated self capacitance is always less than the analytical result, and is expressed here as a percentage of the analytical result, ranging from $7 \%$ to $34 \%$. Since the self capacitance is found to be different by a factor ranging from $3 \mathrm{x}$ to $14 \mathrm{x}$, we would expect significant differences in device behavior. However the difference is much less dramatic if we look instead at the total capacitance of the islands, which also includes the mutual capacitances. In panel (b) of figure 3.10 we show the total capacitance as calculated by the finite element method divided by the total capacitance as calculated by the analytic approximations. Values are again presented as percentages. In every case, the total capacitance is less, but here the ratio ranges from about $59 \%^{1}$ to about $89 \%$, less than a factor of $2 \mathrm{x}$ at most. Therefore we expect the impact on calculated device properties to be much more modest than what would be estimated based on the self capacitances alone.

In figure 3.11 we show the current-voltage properties calculated for this same device. The calculations utilizing the analytic approximations are shown in red, and the calculations utilizing the FEM capacitances are in green. The dominant features, notably the threshold voltage $V_{t h}$ and the effective resistance, are captured reasonably well with the analytic approximations. Meanwhile the detailed structure is significantly different from the more accurate FEM calculations. In particular, the width and height of the Coulomb staircase steps are each overestimated by roughly an order of magnitude. This demonstrates the need for accurate capacitances in these

\footnotetext{
${ }^{1}$ The capacitance ratio is even lower for the end-most islands, but here we have used a disk-like model for the electrodes in the finite element calculation, and a sphere model for the electrodes in the analytic approximations.
} 

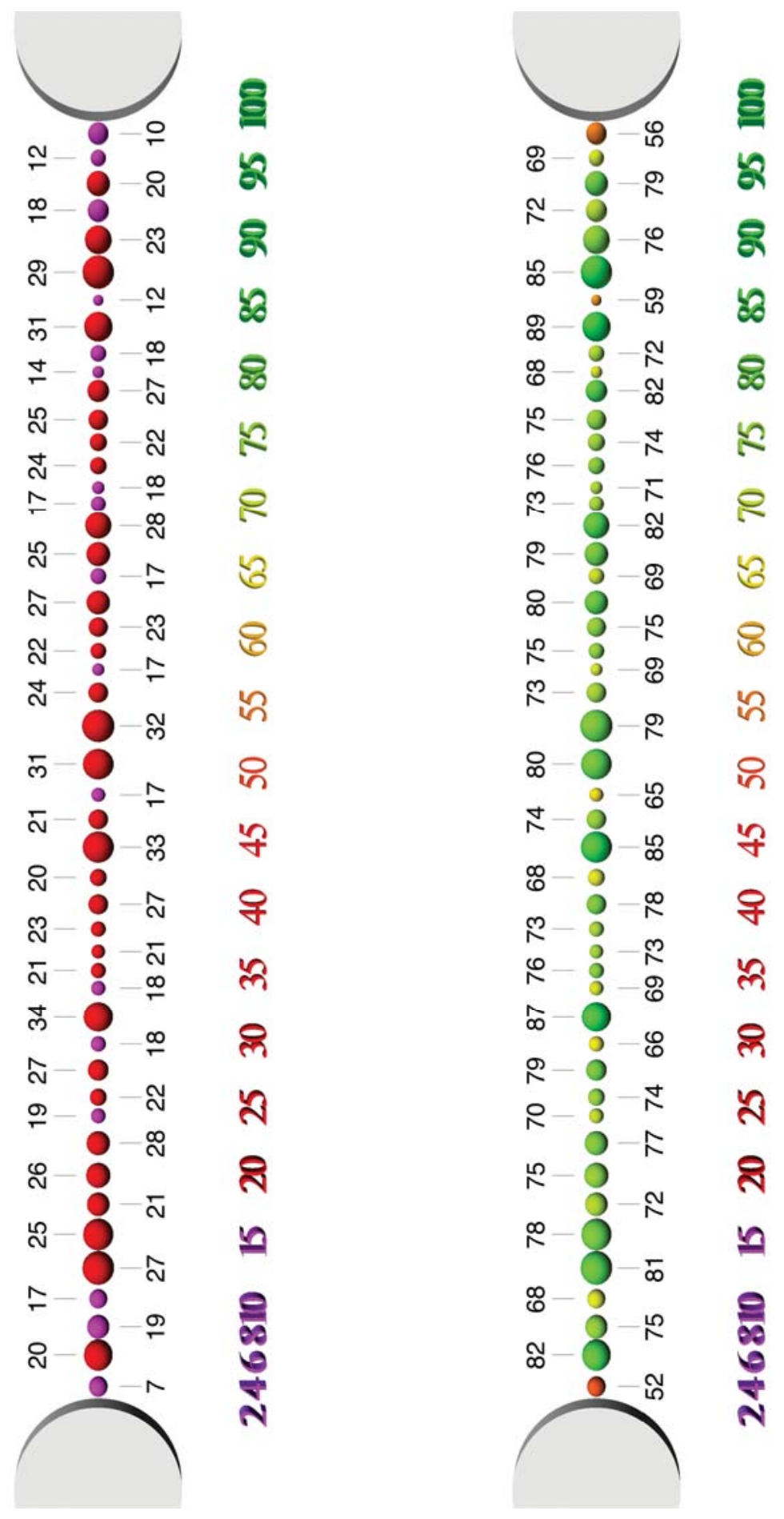

Figure 3.10: Panel (a) shows the self capacitance of each spherical island as a percentage of the self capacitance of a similarly sized isolated sphere. Panel (b) shows the total capacitance of each island as calculated with the finite element method divided by the total capacitance of the island as calculated by analytic approximations, with values expressed as a percentage. 

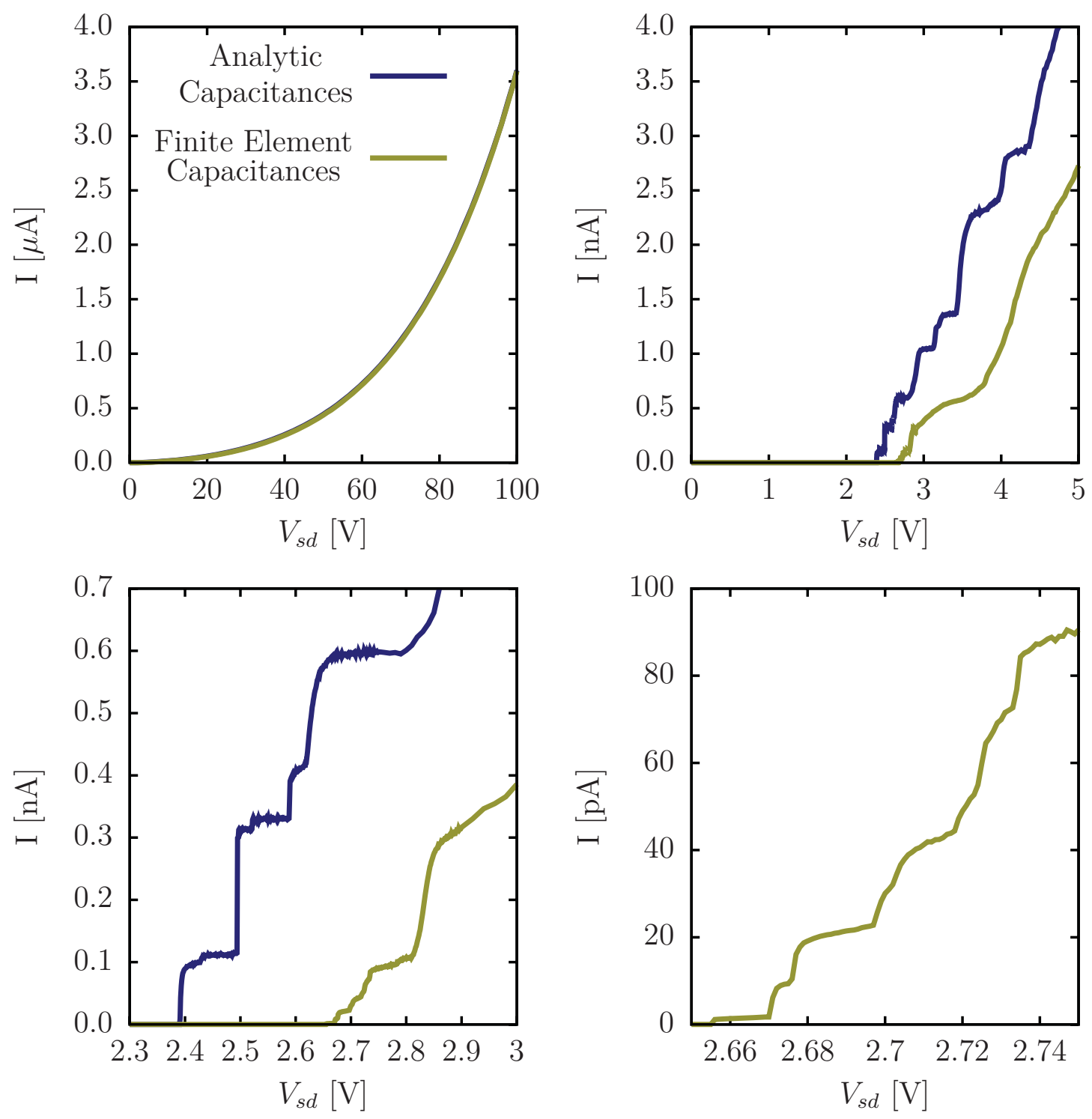

Figure 3.11: Current-voltage properties are calculated for a onedimensional Coulomb blockade device with 50 tunnel junctions. In one case analytic results are used to approximate the capacitances in the system (purple lines). In the other, the capacitances are all calculated using the finite element method (green lines). The dominant features, notably the threshold voltage $V_{t h}$ and the effective resistance, are captured reasonably well with the analytic approximations. Meanwhile the detailed structure, particularly the width and height of the Coulomb staircase steps, are significantly different from the more accurate FEM calculations.

complicated systems. 


\subsubsection{Bent Chain}

In recent work by Hao et al. (Flexible Channels for Tunneling Field Effect Transistors by Quantum Dots Functionalized Boron Nitride Nanotubes. Boyi HaO, Anjana Asthana, Paniz Khanmohammadi Hazaveh, Paul L. Bergstrom, Douglas Banyai, Madhusudan A. Savaikar, John A. JASZCZAK, YOKe KhIN YAP. in manuscript, 2014.) [58], the current is measured in a QDs-BNNT similar to that of Lee et al. (featured in chapter 2 and section 3.5.1) as it is bent to differing degrees. In three different configurations the radius of curvature is approximately $\infty$ (not bent), $500 \mathrm{~nm}$, and $250 \mathrm{~nm}$.

We model this system using a similar system to that of the previous section, with source and drain electrodes, 49 islands, and vacuum separating them. However, instead of the islands being aligned along their centers, we align them along one side as if they are affixed to a nanotube. We use the nanotube to constrain the geometry, but it is not included in the capacitance calculations. We consider the islands to be affixed to a nanotube that is $10 \mathrm{~nm}$ in diameter, and bend the system such that the length of the center of the nanotube is fixed while the top of the tube is stretched and the bottom of the tube is compressed.

In the top panel of figure 3.12 we show the system bent to a radius of curvature of $\infty$ (not bent, black), $+1.5 \mu \mathrm{m}$ (blue), and $-1.5 \mu \mathrm{m}$ (red), where positive curvature indicates bending such that the distance between the islands is reduced, and negative curvature indicates bending such that the distance between the islands is increased.

The bottom panel of figure 3.12 shows the low-bias current for the not-bent system (black) and the system bent to $+500 \mathrm{~nm}$ (blue) and $-500 \mathrm{~nm}$ (red). In order to isolate the effects of the geometry on the electrostatics of the system, the capacitances are 


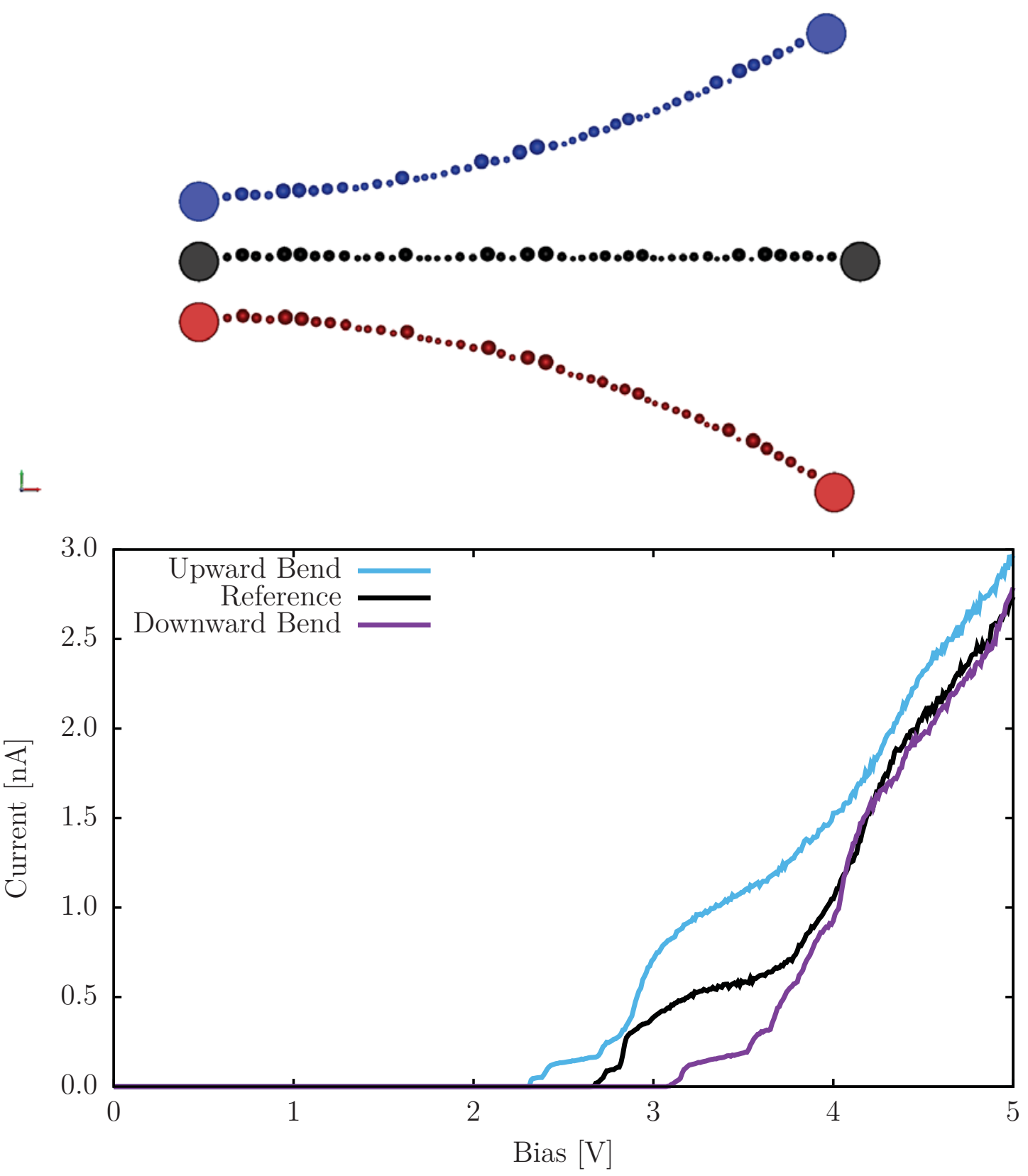

Figure 3.12: A model system representing the bent QDs-BNNTs of Hao et al. [58] in three different bending configurations. The top panel shows the geometry of the bent systems with a radius of curvature of $1.5 \mu \mathrm{m}$, while the $I-V$ simulations are performed with a larger bend of radius $0.5 \mu \mathrm{m}$ to match the experimental conditions. The resistances are calculated based on the geometry of the non-bent device in order to isolate the effects of the changes in capacitance on the device characteristics. For the first device (blue) we see a decrease in the threshold voltage that agrees with the expected increase of the island total-capacitances that comes from decreasing the inter-island spacing. For the second device (red), we see the expected opposite behavior. 


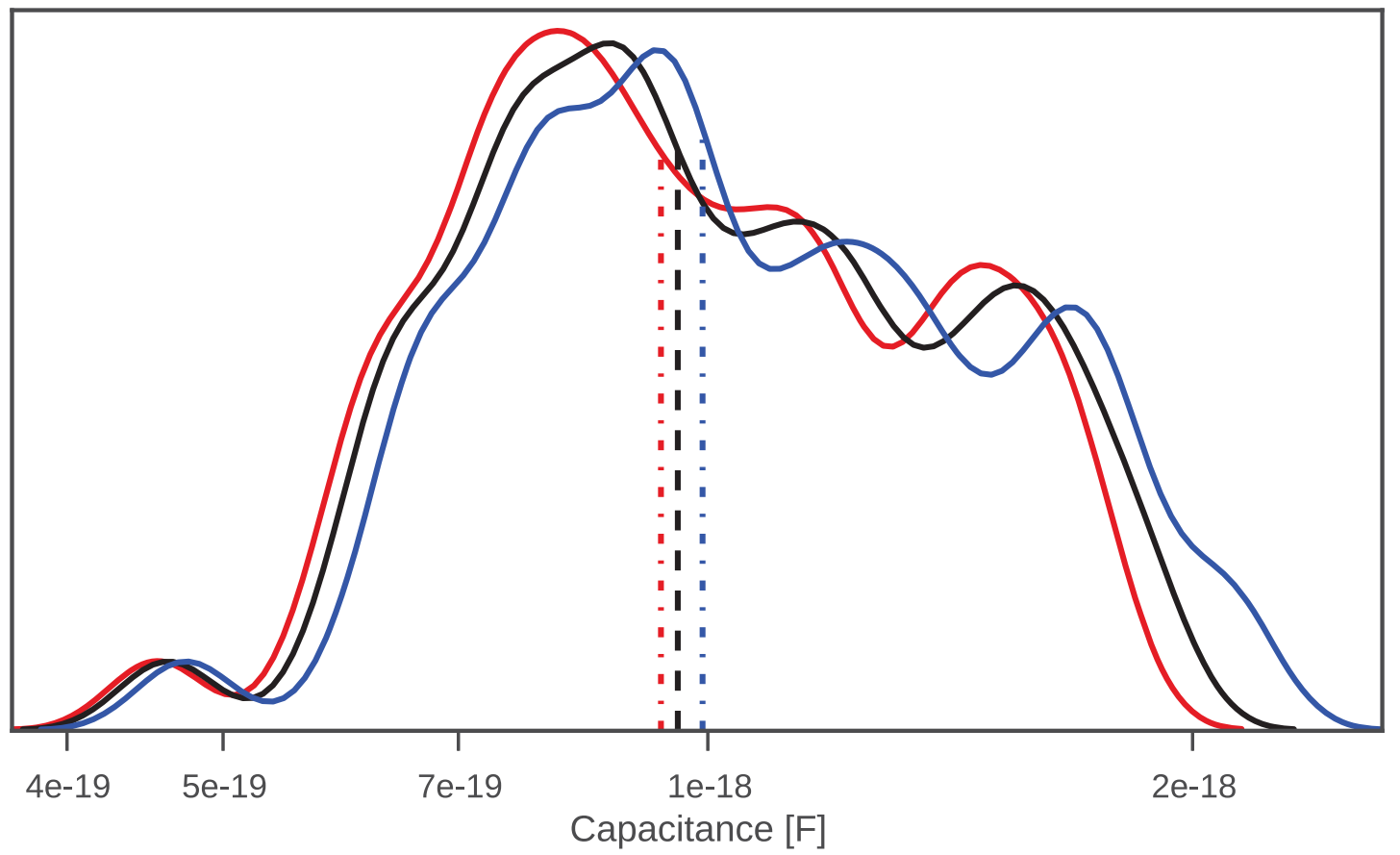

Figure 3.13: The distribution of capacitances increases or decreases relative to the non-bent device (black) as it is bent upwards (blue) or downwards (red), respectively. Here the kernel density estimate is shown as solid lines, and the median capacitance is indicated with dashed lines.

calculated with the changing geometry, while the resistances are calculated from a fixed geometry that corresponds to the not-bent system.

Bending the device 'upward' causes the inter-island separations to decrease, which increases slightly the capacitances in the system as we saw in section 3.4.3. This leads to a slight decrease in the threshold voltage $V_{t h}$ and a modification of where the Coulomb steps are. Bending in the opposite direction leads to the expected slight increase in $V_{t h}$ and again changes in the Coulomb steps. These changes in the capacitances are found to not influence the behavior of the device at higher biases.

In figure 3.13 we show how the distributions of total capacitances change as the system is bent, by calculating the kernel density estimate for the not-bent (black), 
upward bent (blue), and downward bent (red) systems. The median total capacitance values for these systems are 0.96, 0.99, and $0.94 \mathrm{aF}$, respectively, reflecting a change of $+3.6 \%$ or $-2.4 \%$ as the system is bent. The threshold voltage $V_{t h}$ for these systems are $2.7,2.3$, and $3.1 \mathrm{~V}$, respectively, which makes for a much more significant change of $-16 \%$ or $+13 \%$ as the system is bent. It is interesting to note that for this system we cannot accurately predict the change in threshold voltage $(-16 \% /+13 \%)$ from either the change in median charging energy $(-3.5 \% /+2.4 \%)$ or from the maximum change in an individual island's charging energy $(-12 \% /+7.8 \%)$, which both underestimate the change. This unpredictability has been previously remarked upon in section 2.5 and in references $[1,3]$.

\subsubsection{D Systems}

Karre et al. [28, 37] have developed Coulomb blockade transistors capable of room temperature operation where the conduction channel consists of a field of randomly deposited tungsten quantum dots. Simulation of these devices is discussed in section 2.4 .2 .

The method described in this chapter was developed so that we would have the ability to more accurately simulate these devices, since non-nearest neighbor capacitance and self capacitance cannot be reasonably approximated without numerical methods. To illustrate the necessity of an improved capacitance calculation scheme, we compare the calculated self-capacitances to the analytic approximation for the self-capacitance of a sphere of the same size. In figure 3.14 the islands in our simulated system are colored according to the ratio of self-capacitance to analytic self-capacitance. The density of the islands has a huge impact on the self-capacitance. The average island has a self-capacitance approximately $3 \%$ of the analytic value, while islands on the 
edges of the field have values that range from approximately $10-15 \%$. This is a much larger difference than we saw in the one-dimensional chain (section 3.5.1), as would be expected.

\subsection{Conclusions}

We developed a method for calculating capacitances using the finite element method (FEM) which utilizes several open source projects that were not previously compatible. We developed a work flow that integrates this method with the recently developed Multi-Island Transport Simulator (MITS) in order to improve our ability to model complex Coulomb blockade devices with capacitances that are not well described by analytic approximations. 


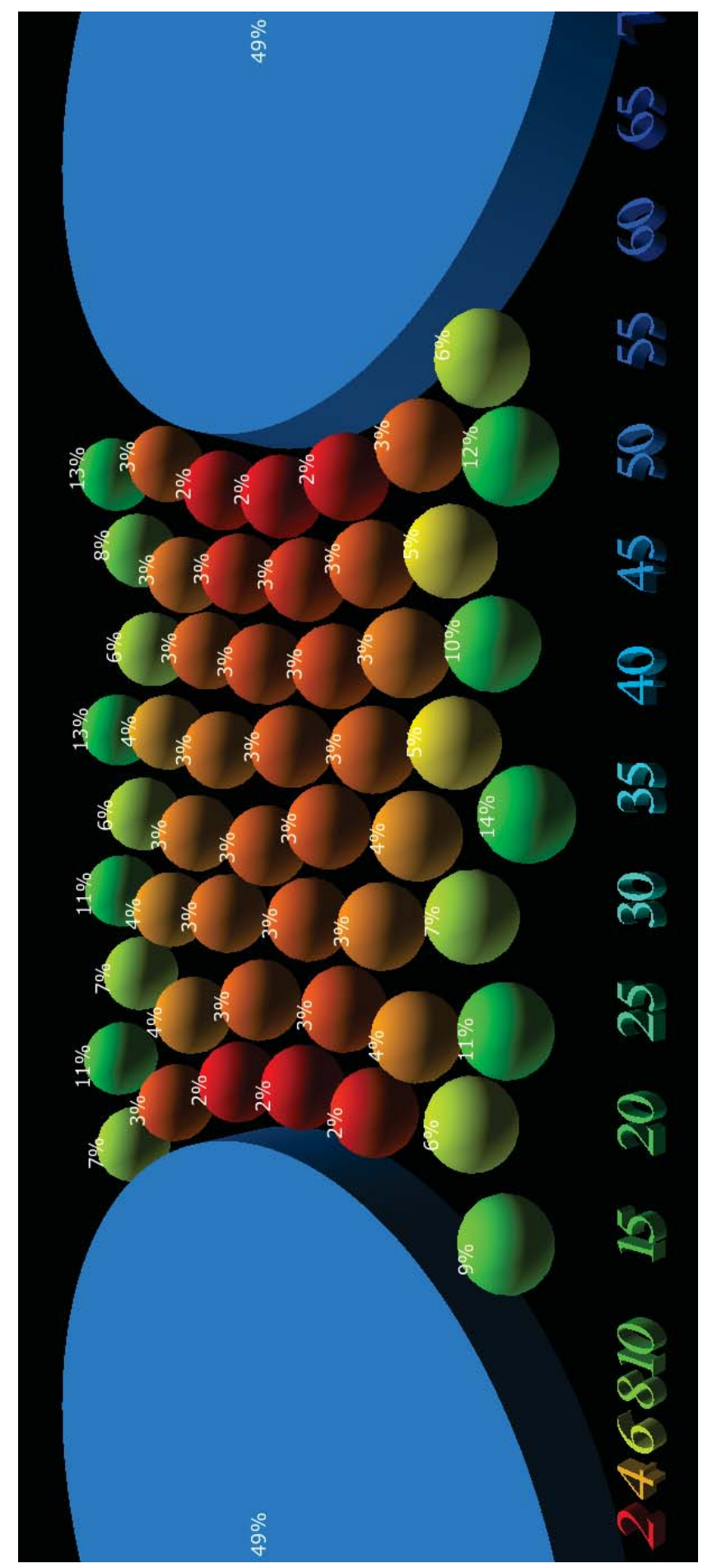

Figure 3.14: The self capacitance of the sphere as a percentage of the self capacitance of a similarly sized isolated sphere. 



\section{Chapter 4}

\section{Tunneling Resistance Between Clusters}

In our investigations of the conduction behavior of Coulomb blockade devices, and especially work simulating scanning tunneling microscopy, quantum mechanical tunneling of electrons is a core component of these physical systems. In this chapter we discuss tunneling from several perspectives, and calculate tunneling properties by analytic and computational means. These preliminary studies prove the feasibility of our approach, and also provide insight into some potential hazards of the applied electronic structure methods.

\subsection{Introduction}

In the simulation of scanning tunneling microscopy and Coulomb blockade devices, it is essential to have a good grasp of the underlying tunneling phenomena occurring in the devices. Most obviously, the magnitude of the resulting current is dependent 
upon the effective resistance of the tunneling junctions. More subtly, differences in the relative resistance of the tunnel junctions in a Coulomb blockade device affect the relative heights of the Coulomb staircase structure. This tunneling resistance is exquisitely dependent upon the geometric details of the junction. For example, in a typical junction, increasing the junction width by just one nanometer increases the tunneling resistance by an astounding ten orders of magnitude. This is a result that is readily available from the most basic theoretical treatments of tunneling, and is borne out in experiment. Less well studied is how other elements of geometry, such as curvature and roughness, impact tunneling. In this chapter we employ modern quantum chemistry methods to investigate this very question.

\subsection{Effect of Resistances on Coulomb Blockade Devices}

For a quick demonstration of the importance of resistances to the properties of Coulomb blockade devices, we calculated current-voltage characteristics using a test system with randomly distributed resistances. The test system is the same as for the current-voltage measurements in chapter 3, using capacitances calculated with the finite element method (FEM), and tunneling resistances calculated as described in chapter 2 .

In order to produce the randomized resistances, we supplied the resistance subroutine in MITS with sham junction widths that had been selected from a uniform distribution. Three different uniform distributions were sampled, with ranges $(0, s),\left(\frac{1}{2} s, \frac{3}{2} s\right)$, and $(s, 2 s)$, where $s$ is the maximum junction width originally present in the system. The tunneling resistances depend exponentially on the junction widths, and so the resulting resistances have an exponential distribution. 


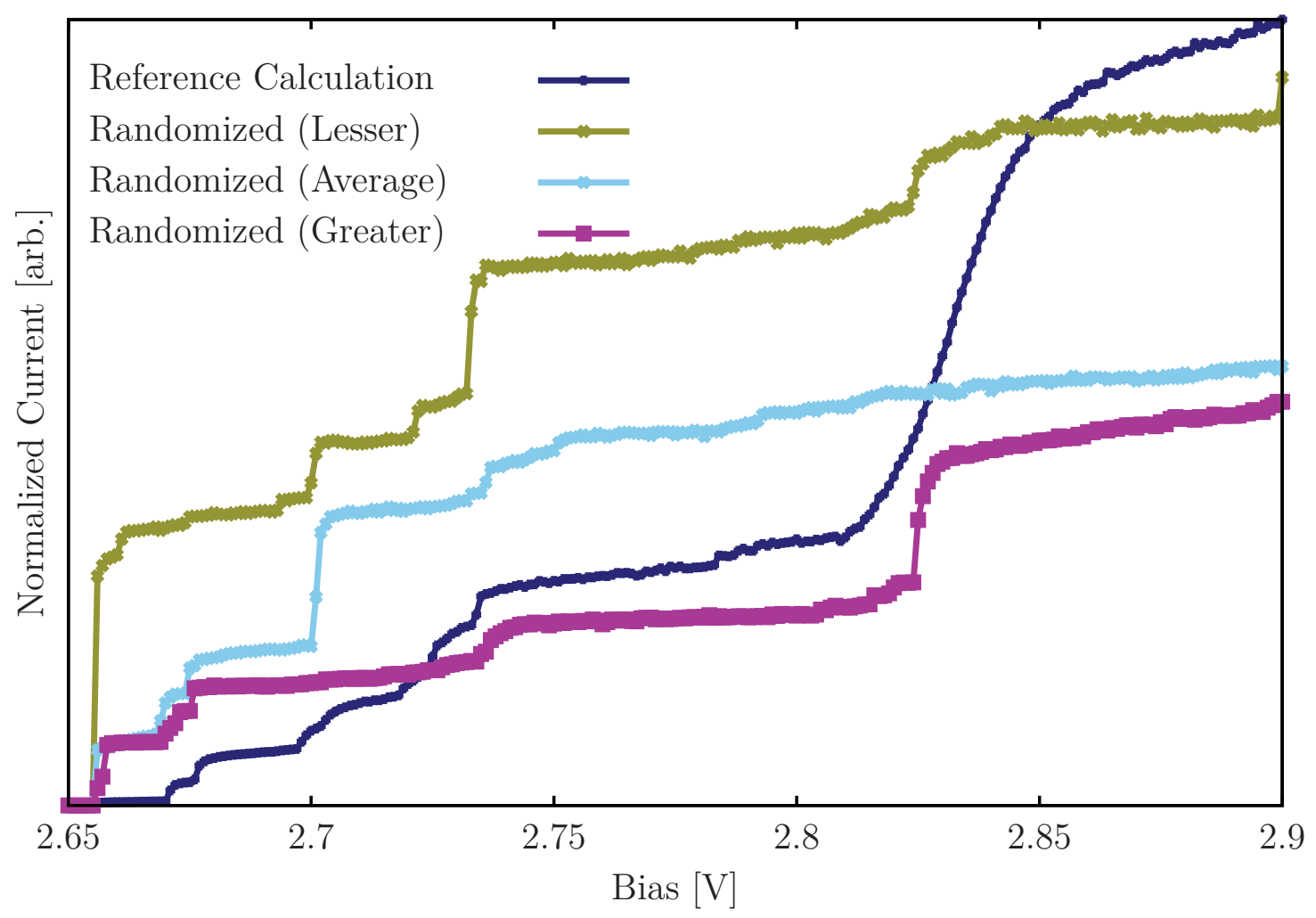

Figure 4.1: Normalized current-voltage characteristics are calculated for a system with randomized resistances. The reference system (purple) is the same 1D SET as in chapter 3. Three randomized systems are simulated with average resistances that are lesser than (green), greater than (fuchsia), or similar to (blue) the average resistance in the reference system.

Figure 4.1 shows the low-bias current calculations for the reference system and the three randomized systems. The results vary by six orders of magnitude, so in this figure the current has been normalized by dividing by the magnitude of the current at $+5 \mathrm{~V}$.

At this voltage scale, the most interesting features are the threshold voltage and the height and positions (voltages) of the Coulomb steps. The threshold voltage is determined entirely by the energetics of the system, which is a function of the capacitances but not the resistances, and so it does not change. As expected, the relative heights of the steps change as the resistances are randomized, creating qualitatively different 
results. The voltages of the individual Coulomb steps are not expected to depend on the resistances, and for the most part this expectation holds. Most interestingly however, the existence of each individual step depends on the resistances, and at 2.70 $\mathrm{V}$ and again at $2.82 \mathrm{~V}$ we see steps that show up in some systems and not in others. Specifically with the reference calculation (purple line in Figure 4.1) we see a very large step just beyond $2.8 \mathrm{~V}$, while the randomized system with similar resistances (blue line) exhibits no such steps. Meanwhile this same system (blue line) shows a dramatic step at $2.70 \mathrm{~V}$ which is much more subdued in the reference calculation and essentially absent from the "greater resistance" system (fuchsia line).

With this we have shown that in addition to determining the overall magnitude of the current, the qualitative details of the tunneling current are sensitive to the individual junction resistances.

\subsection{Tunneling Through Thin Films}

One of the few tunneling calculations that can be evaluated analytically is for tunneling through a one-dimensional rectangular barrier. If we can calculate the ratio $T$ of the amplitudes of the incident and transmitted wavefunctions, then we can calculate the probability of an incident electron tunneling through the barrier, $P=|T|^{2}$. The transmission function $T$ is

$$
T=\left[1+\frac{\phi^{2} \sinh ^{2}(k d)}{4 E(\phi-E)}\right]^{-1},
$$

where $E$ is the energy of the incoming electron, $\phi$ is the height of the barrier, $d$ is the width of the barrier, and

$$
k=\sqrt{\frac{2 m_{e}(\phi-E)}{\hbar^{2}}}
$$


is the wavenumber of electron in the barrier.

If the barrier is not of a constant height, the WKB approximation can be used to calculate the transmission through the barrier:

$$
T=\exp \left\{-2 \int_{0}^{d} d x \sqrt{\frac{2 m_{e}}{\hbar^{2}}(\phi(x)-E)}\right\}
$$

Interestingly, in the case of a constant barrier height, this simplifies to

$$
T=e^{-2 d \sqrt{\frac{2 m_{e}}{\hbar^{2}}(\phi-E)}},
$$

and in this approximate form the exponential dependence on the barrier width $d$ is clearly identified.

\subsubsection{Tunnel Barriers from Finite Element Method Calculations}

In section 3.4.4 we calculated the potential energy barrier profile for a model system that consisted of three spherical islands separated from each other and from two larger electrodes by $1 \mathrm{~nm}$ tunnel junctions. The barrier profiles are shown in figure 3.9.

For each of these four barriers and for a trapezoidal barrier we calculated the transmission probability for an electron coming at the barrier from the left side to tunnel through to the right side. We evaluated the WKB expression for transmission probility in eq. 4.3 by numerical integration. We assumed the incoming electron had an energy equal to the Fermi level on the left side of the barrier. The work function (the value of $\phi(x)$ at the edge of the barrier) was taken to be $5 \mathrm{eV}$. The barriers are $1 \mathrm{~nm}$ wide and drop by $1 \mathrm{eV}$ from left to right. 
The 'area' of each barrier (the value of the integral in the exponent of eq. 4.3) is less than that for the trapazoidal barrier by $4 \%, 6 \%, 3 \%$, and $1 \%$ for the first through fourth junctions, respectively. The value of the transmission probability, and by extension the value of the tunneling current, is greater than that for the trapezoidal barrier by $14 \%, 22 \%, 11 \%$, and $4 \%$ for these same junctions.

\subsection{Tunneling Between Nanoscale Particles}

Next we investigate the effect of extreme curvature on the effective resistance of a tunnel junction by calculating the conductance properties of tunnel junctions between small clusters of gold atoms, representing nanoscale gold particles. We begin with spherical cage-like clusters of 32 gold atoms, and progress to hexagonal 6 atom gold clusters, and finally single atom gold 'clusters.'

We use a Green's function approach to calculate the current through our model systems. A density functional theory (DFT) approach is used to calculate the ground state electronic structure.

\subsubsection{Density Functional Theory}

We use density functional theory to find the ground state geometry for the metal clusters. To do so, we find the ground state electronic structure for a test configuration, and calculate the forces on the ions. The geometry is optimized until the forces on the ions are below a threshold value.

Under the Born-Oppenheimer approximation [59] we can consider the electronic and 
ionic degrees of freedom to be uncoupled, and we can find the electronic ground state subject to a potential due to the fixed ions.

Calculating the ground state electronic structure begins with describing the system with the Schrödinger equation,

$$
H \Psi=E \Psi
$$

where the electronic Hamiltonion in atomic units is

$$
H=-\sum_{i=1}^{N} \frac{1}{2} \nabla_{i}^{2}+\sum_{i=1}^{N} \sum_{j=i+1}^{M} \frac{1}{\left|\overrightarrow{r_{i}}-\overrightarrow{r_{j}}\right|}-\sum_{i=1}^{N} \sum_{A=1}^{M} \frac{Z_{A}}{\left|\overrightarrow{r_{i}}-\overrightarrow{r_{A}}\right|}
$$

Here $N$ is the number of electrons, $M$ is the number of ions, $Z_{A}$ is the atomic number of ion $A, \overrightarrow{r_{i}}$ is the position of electron $i$, and $\overrightarrow{r_{A}}$ is the position of ion $A$. The first term in the Hamiltonian is the kinetic energy of the electrons, the second term is the repulsion between electrons and electrons, and the last term is the attraction between the ions and electrons. The total energy of the system is then

$$
E_{\text {total }}=E+\sum_{A=1}^{M} \sum_{B=A+1}^{M} \frac{Z_{A} Z_{B}}{\left|\overrightarrow{r_{A}}-\overrightarrow{r_{B}}\right|}
$$

where the second term here is the Coulomb repulsion of the ions.

The Hohenberg-Kohn theorem [60] states that the all observables of a quantum system can, in principle, be derived from the electron density of the system. This reduces the $3 N$-dimensional problem of finding the $N$ wavefunctions to the 3 -dimensional problem of finding the ground state electron density. The energy of the system is now described by

$$
E[\rho]=T[\rho]+V_{N e}[\rho]+V_{e e}[\rho]=T[\rho]+V_{N e}[\rho]+V_{H}[\rho]+V_{X C}[\rho]
$$

where $T[\rho]$ is the electron kinetic energy term, $V_{N e}[\rho]$ is the electron-ion interaction 
term, and $V_{e e}[\rho]$ is the electron-electron interaction term. This electron-electron interaction term now includes the Hartree potential, $V_{H}[\rho]$, which is the electron-electron repulsion, as well as the exchange-correlation potential, $V_{X C}[\rho]$, which includes the exchange interaction and all the correlation effects between electrons.

We use DFT as implemented in the Gaussian 09 software package [61]. We use the B3LYP [62] hybrid exchange and correlation functional. The LanL2DZ basis set [6365] was used, which combines an effective core potential (ECP) pseudopotential to represent the core electrons, with the Dunning/Huzinaga full double zeta basis set (D95) [66] to represent the valence electrons. Self consistent field calculations were performed until the density met a convergence criteria of $10^{-7}$.

\subsubsection{Geometry}

Each model system consists of two gold clusters attached to source and drain electrodes. In the DFT calculations, the ends of the electrodes are represented by an atomic wire consisting of two gold atoms. Each cluster is attached to a separate electrode via a sulfur linker atom (see Figure 4.2). The sulfur atoms, the clusters, and the vacuum between them constitute the scattering region for the purposes of the Green's function conductance calculation. The Au-Au bond length in the electrode and the Au-S bond length between the electrode or cluster and the linker S atom were fixed at 2.57 and $2.35 \AA$, respectively, a value corresponding to the bond length in the isolated $\mathrm{Au}-\mathrm{Au}$ or $\mathrm{Au}-\mathrm{S}$ dimer.

The cage-like $\mathrm{Au}_{32}$ and hexagonal $\mathrm{Au}_{6}$ clusters were both optimized until the residual forces were less than $0.01 \mathrm{eV}^{-1}$. These clusters were then frozen and attached to the electrodes without further optimization. The distance of closest approach between 

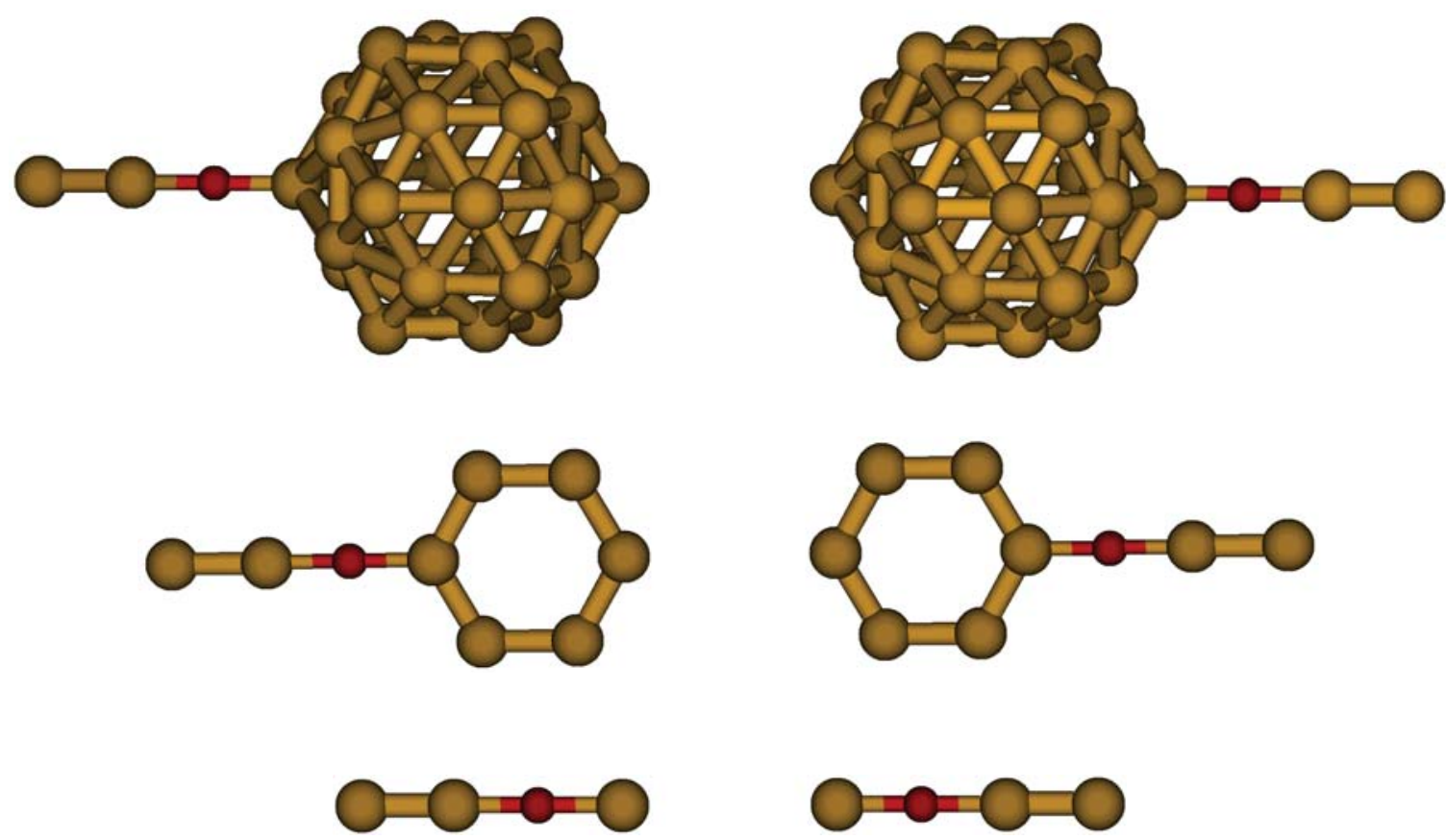

Figure 4.2: Tunneling between clusters was investigated using three different models for clusters of various sizes. The largest clusters we used were spherical cage-like clusters of 32 gold atoms. Our middle size clusters consist of a ring of 6 gold atoms. The smallest possible cluster was modeled with a single gold atom. In each case, the clusters were connected to a gold electrode via a sulfur linker atom. Each of these clusters are shown with a distance of closest approach of $6 \AA$, which results in a tunneling barrier approximately $3.5 \AA$ wide.

the clusters was systematically varied from 1.5 to $6 \AA$ for the larger $A_{32}$ clusters, and from 2 to $9 \AA$ the smaller $\mathrm{Au}_{6}$ and single atom clusters. Figure 4.2 shows the three systems with a distance of $6 \AA$ between them, which results in a tunneling barrier with a width of approximately $3.5 \AA$.

\subsubsection{Results}

In order to compare the cluster results to the simple analytic results, it would be useful to know the height and width of the tunneling barrier between the clusters. 


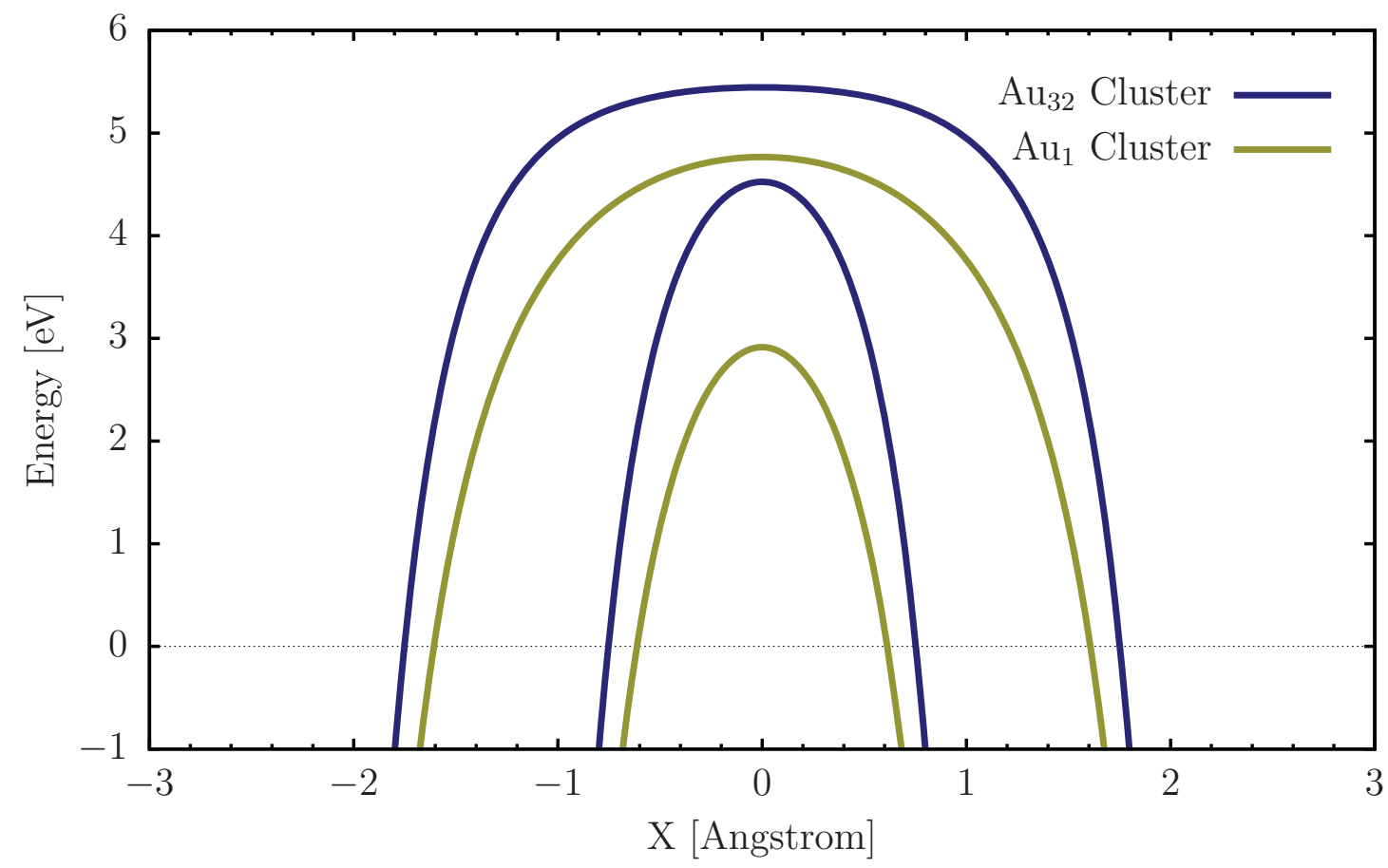

Figure 4.3: The profile of the tunneling barrier is shown for larger $\mathrm{Au}_{32}$ cluster (purple) and the minimal single-atom 'cluster' (green) for two different separation distances. $6 \AA$ separation (solid lines) means the closest atoms are at $\pm 3 \AA$, while $4 \AA$ separation (dashed lines) means the closest atoms are at $\pm 2 \AA$.

Figure 4.3 shows the calculated profile of the tunneling barrier for the $\mathrm{Au}_{32}$ cluster and the single-atom systems. The solid lines show the profile for the barrier for a cluster separation of $6 \AA$, which means the closest atoms are at $\pm 3 \AA$. We find the barrier to be approximately $3.4 \AA$ wide and $5.0 \mathrm{eV}$ high for the $\mathrm{Au}_{32}$ cluster, and 3.0 $\AA$ wide and $4.5 \mathrm{eV}$ high for the single-atom system. Reducing the separation by $2.0 \AA$ (dashed lines) reduces the width of the barrier by $2.0 \AA$, as expected, but also reduces the barrier height, with the single-atom system more dramatically affected than the $\mathrm{Au}_{32}$ system. From this we find we can relate separation distances to barrier width by subtracting 2.6 (3.0) $\AA$ from the separation distance for the $\mathrm{Au}_{32}$ (single-atom) system. 


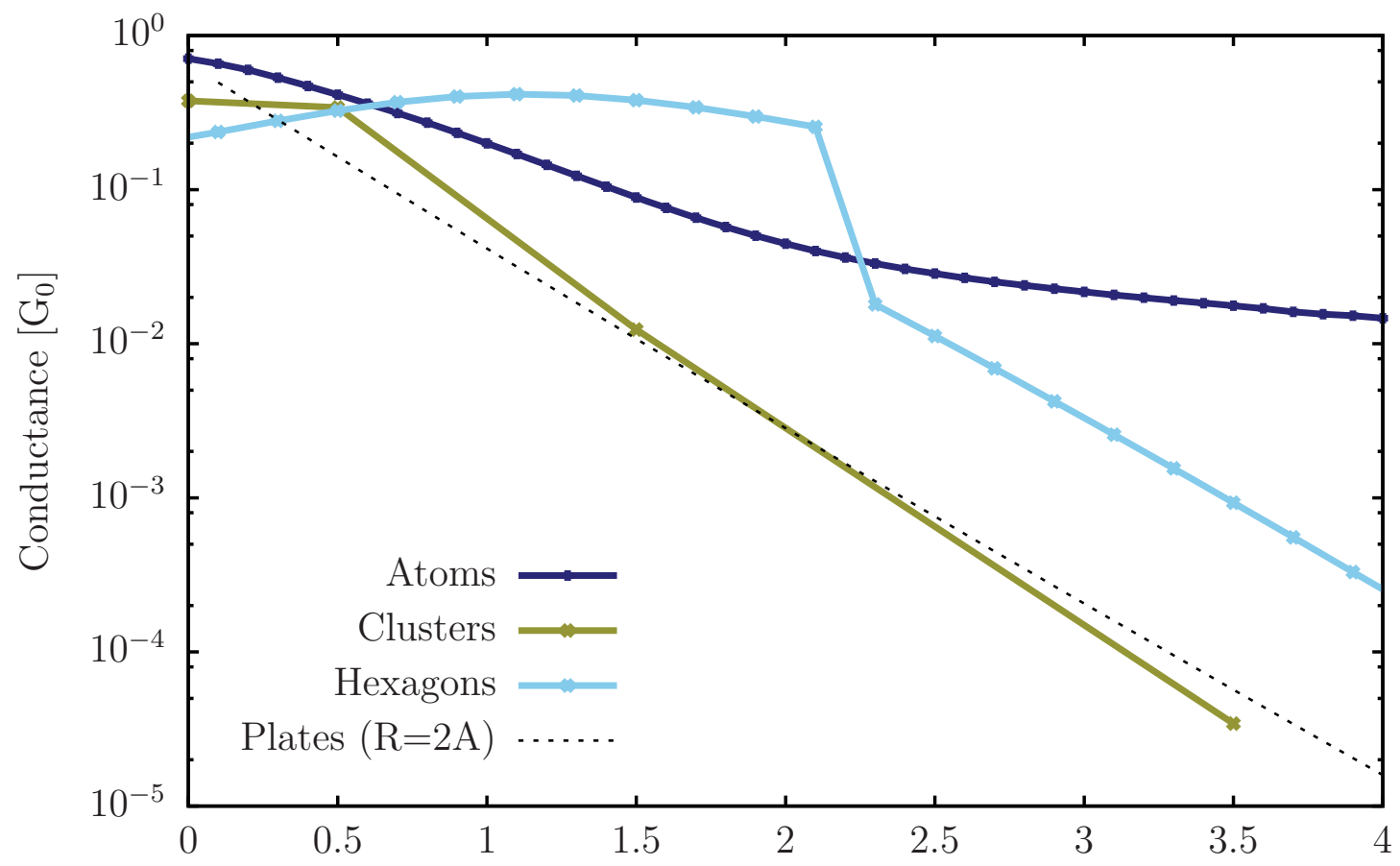

Figure 4.4: Conductance is calculated as a function of junction width for four different systems. The analytic 1D barrier (dashed line) shows a simple exponential decay with junction width. The $\mathrm{Au}_{32}$ clusters (green) show a very similar exponential decay. The single-atom system (purple) does not show the same exponential decay, even for moderately large junction widths. Finally the $\mathrm{Au}_{6}$ clusters (blue) show a surprising discontinuity in the conductance data.

Figure 4.4 shows the calculated conductance for each of the cluster systems as a function of barrier width. For comparison, the analytic solution is also shown for a one-dimensional barrier with $5 \mathrm{eV}$ workfunction and an area of $4 \pi \AA^{2}$ (red line). Few data points were calculated for the $\mathrm{Au}_{32}$ system due to the computational cost for this larger system, but from the data we do have we can see the tunneling conductance follows a similar exponential decrease with barrier width as the 1D system shows. The hexagonal clusters show a discontinuous behavior that we will explain in the next section. In the mean time, we will note that for larger barrier widths the hexagonal system shows a very clean exponential decay matching the behavior of the 1D system, albeit with a higher conductance that would correspond to a 1D 
system with a significantly greater area. With the single-atom system we finally get to a system too small to exhibit similar behavior to the 1D case, as the conductance decreases at a rate that would correspond to a $1 \mathrm{D}$ system with a very low tunneling barrier height (workfunction).

\subsubsection{The Hexagon Cluster Mystery}

We initially expected the discontinuity in the hexagonal system data to be indicative of an error, and were pleased to discover instead an interesting bit of physics.

We began our inspection by looking at the self consistent field (SCF) energy of the system as a function of separation distance (panel (a) in figure 4.5). At $4.7 \AA$ we found a discontinuity in the slope. Starting from $4.6 \AA$, we increased the separation distance and recalculated the electronic structure using the electron densities from the previous calculation as a starting guess. By this method, we were able to map out a series of states (labeled "State 1") for separation distances greater than $4.7 \AA$ that are not the ground state, but rather local minima with the same symmetries as the bonded $(<4.7 \AA)$ system. Similarly, we were able to find solutions below $4.7 \AA$ that were similar to the ground state for the distant systems ("State 2"). Interestingly, below $3.4 \AA$, calculations starting from state 2 find a lower energy state, which is also not the ground state. Panel (a) in figure 4.5 shows the energy of the states we found for separation distances ranging from 2.2 to $9 \AA$.

In panel (b) of figure 4.5, we have calculated the tunneling conductance for each of the states found in panel (a). There is a clear difference in the behavior of the conductance depending upon the electronic state, with the bonded state maintaining a high conductance for large separations, while the non-bonded state has an exponential 

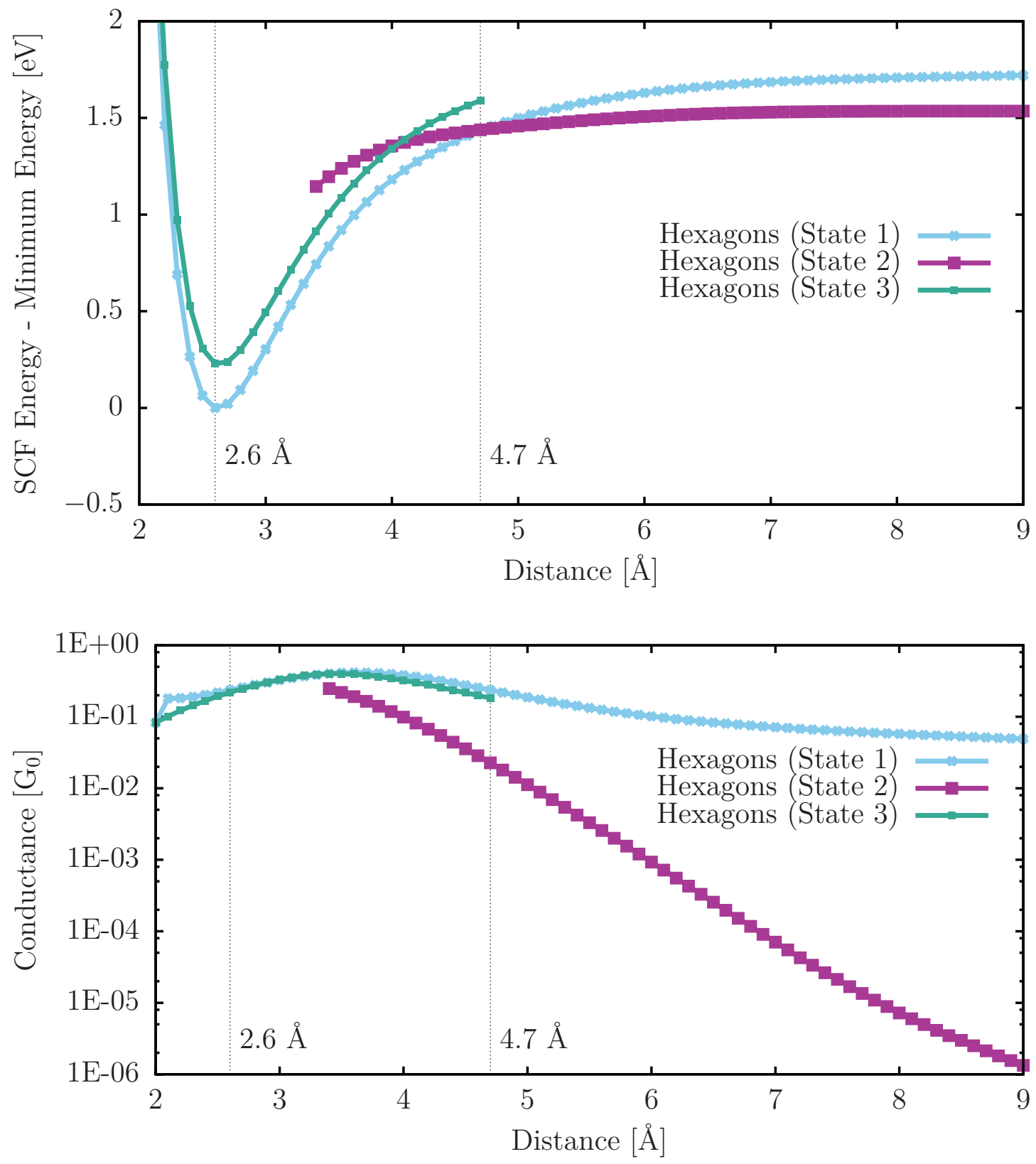

Figure 4.5: The hexagonal system can be coerced into different local minima electronic states. The 'bonded' state (blue), which is the ground state when the separation distance is minimal (upper panel), shows a tunneling conductance that doesn't decay with distance in the expected manner (lower panel). The 'non-bonded' state (fuchsia), which is the ground state at larger separations, does show a simple exponential decay of conductance with increasing separation distance as expected from simple models. A third state (green) has conductance characteristics similar to the non-bonded state, but has a lower energy at small separation distances. 


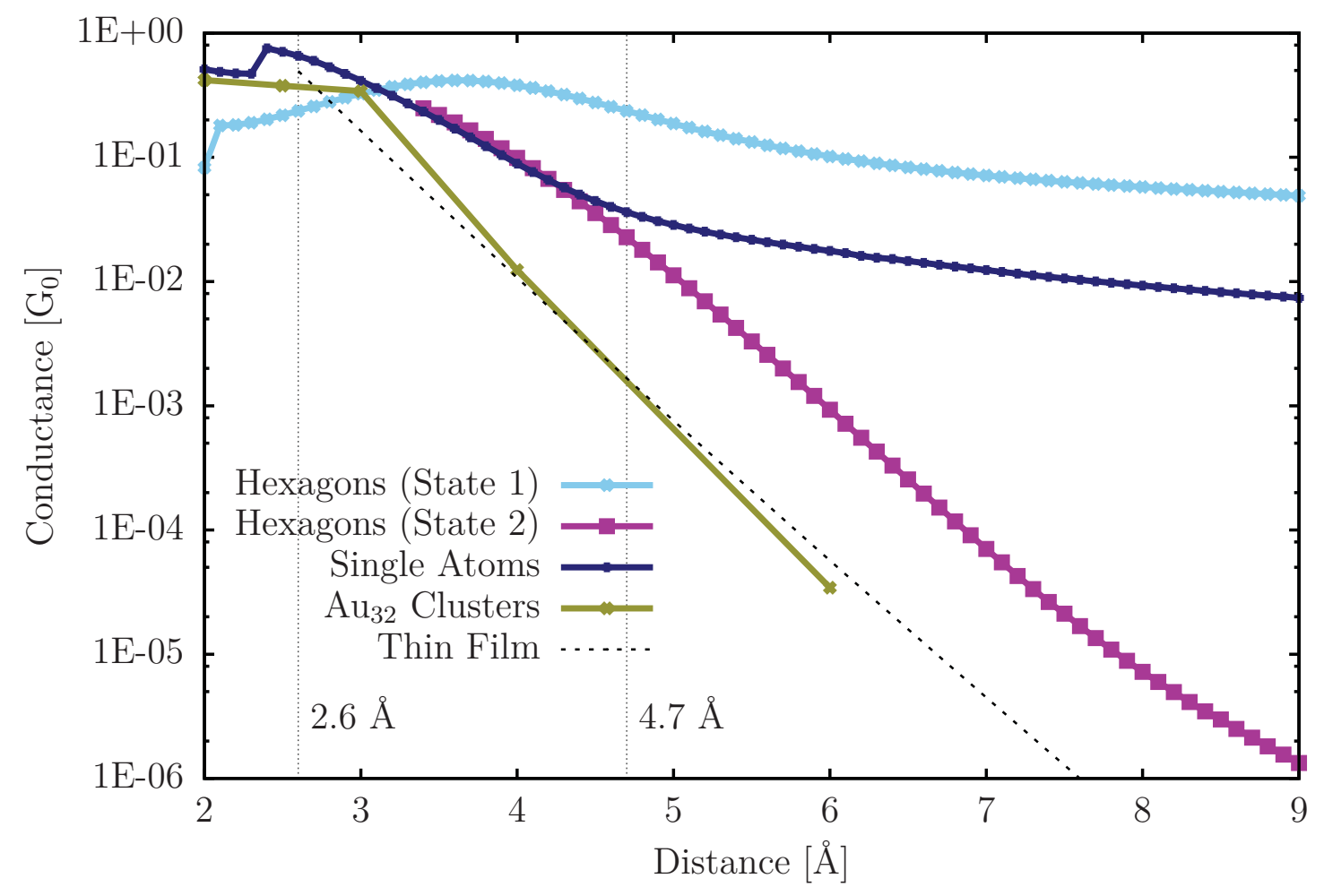

Figure 4.6: Conductance is calculated as a function of distance for each of the investigated systems. The large $\mathrm{Au}_{32}$ cluster (green) and the 'nonbonded' electronic state of the $\mathrm{Au}_{6}$ cluster (fuchsia) show an exponential decrease in the tunneling conductance similar to the 1D tunneling barrier model with a $5 \mathrm{eV}$ workfunction (black dotted). Meanwhile, the 'bonded' state of the $\mathrm{Au}_{6}$ cluster (blue) and the single-atom system (purple) both show anomalously large conductance values at larger distances.

decrease in the conductance similar to the 1D system.

Figure 4.6 shows the conductance for each system. Here we can see that the singleatom system behaves very much like the bonded hexagonal system, while the $\mathrm{Au}_{32}$ system behaves like the non-bonded hexagonal system, which also behaves like the $1 \mathrm{D}$ system. This result seems reasonable in that the $\mathrm{Au}_{32}$ clusters are not bonded and likewise the 1D analytic result assumes there is not bonding across the gap. 


\subsection{Conclusion}

In this chapter we demonstrated the importance of tunneling resistances to the behavior of Coulomb blockade devices of the QDs-BNNT type. We described the analytic model for tunneling through a thin film in order to illustrate the classic exponential dependence on barrier width. We provided a preliminary look at using a Green's function and density functional theory approach to calculate tunneling conductances of junctions between gold nanoparticles, and found a similar exponential decay with distance for the larger particles we simulated. 



\section{Chapter 5}

\section{Simulation of Scanning Tunneling Microscopy and Spectroscopy}

\section{$5.1 \quad$ Introduction}

Since the development of the scanning tunneling microscope (STM) in 1981 [21], many approaches have been developed to simulate STM measurements. Most begin with calculating the local electronic density of states (LDOS) for the sample surface, often within the density functional theory (DFT) framework [67], but with the notable exception of the superposition of atomic charge-density method [68, 69]. In this section we briefly overview current methods for calculating STM properties, from simplistic to sophisticated, and conclude with a description of SLSTM. 


\subsubsection{Methods for computational STM}

The simplest method for simulating STM constant-current images is the atomic charge-density superposition method $[68,69]$. This method has a surprising ability to generate quantitatively reasonable STM images for certain systems as demonstrated by Tromp et al. [69], and is able to handle very large systems due to its mathematical simplicity. A significatn disadvantage of this method is that it fails to provide information about the electronic contribution to the STM images.

One of the earliest, and still one of the most popular methods is the so-called TersoffHamann method, first used in 1983 to investigate the corrugation height of constantcurrent-mode STM micrographs [70]. In this method the electronic structure of the probe tip is ignored and the tunneling current is taken to be proportional to the local density of states of the sample at the position where the probe tip would be. A distinction should be noted between the theory of STM operation as described in Tersoff and Hamann's seminal paper [70] and the much simplified "no-tip-needed" approximation used in that same paper. Advantages of this method include not needing an accurate model of the tip due to the tip-independence, and the ease of calculating constant-current and constant-height images once the local density of states is known. Disadvantages include the lack of inclusion of the effects of the tip, and implementation-specific disadvantages include the need for a larger basis set or higher energy cutoff and better convergence than in typical DFT calculations in order to accurately describe the local density of states (LDOS) in the vacuum. That is to say, calculations that provide suitably accurate results for the DOS in the bulk and at the surface will not necessarily provide accurate results for the LDOS in the vacuum, which in turn will not provide accurate STM results. Furthermore, we know of no implementations of the Tersoff-Hamann method that allow for calculating voltagebias-dependent properties such as current imaging tunneling spectroscopy (CITS) or 
scanning tunneling spectroscopy (STS). A method for calculating STS data based on the theory of Tersoff and Hamann [70] was developed by Lang [71]. This is a fast method for calculating voltage-bias-dependent information for a tip at a fixed position but it has not previously been extended to the calculation of STM images.

A more sophisticated version of the Tersoff-Hamann method starts at the same level of theory as outlined in Tersoff and Hamman's seminal paper [70] but does not discard the effect of the tip. An example is Paz and colleagues' STM simulator implemented in the SIESTA code [72]. In this work they also addressed the difficulty of obtaining accurate results in the vacuum by taking the LDOS near the surface, which SIESTA easily calculates accurately, and projects these values out into the vacuum using a Green's function method. Advantages of this method include the ability to calculate tip-dependent and bias-dependent properties. A significaant disadvantage is limited public availability of software implementing such methods.

The state of the art method for STM/STS image simulation is quantum-transportbased calculations such as the elastic scattering quantum chemistry method [73]. This is the first of the methods described that allows the presence of the tip and the applied bias to affect the electronic structure of the sample. This is the most accurate way to calculate STM properties. Even nuanced effects influenced by directional bonding can be investigated. However, this method requires calculating a self-consistent field (SCF) solution for every combination of tip position and applied voltage. The disadvantages include the dramatically increased computational resources required for such approaches and again a lack a of publicly available software implementing the method.

We introduce a new method that combines the geometry of the atomic charge-densitysuperposition method with the scanning tunneling spectroscopy approach of Lang [71] for a result that is similar in concept to the approach of Paz [72]. This allows for fast 
calculations of STM/STS properties that are not available through the superpositionof-atomic-charge method, the Lang method, or the Tersoff-Hamann method, including current-imaging-tunneling spectroscopy (CITS) images. As with the Paz method, we make use of the LDOS near the surface, avoiding the Tersoff-Hamann method's need for large vacuum regions, increased basis sets, and tighter convergence. This introduces a disadvantage relative to the quantum-transport-based methods in that the calculation of the LDOS in the vacuum is not exact, and the method for calculating the vacuum LDOS from the surface LDOS is less rigorous than in the method of Paz et al.

\subsection{Method}

In this section we describe the mathematical background, approximations, and method implemented in SLSTM. We begin by describing the theories that provide the foundation for the method.

\subsubsection{The STM Theory of Tersoff and Hamann}

One of the most useful and influential formulations of the theoretical tunneling current was first described by Tersoff and Hamann in 1983 [70]. Here the Bardeen expression for tunneling between two surfaces [74] is evaluated for the case where the wavefunction associated with one of the surfaces (the tip) has a spherically symmetric, or s-type characteristic. This allows for the surface integral to be evaluated and the result is an expression for the tunneling current as a convolution of the electronic density of states of the tip, $\rho_{t}(E)$, and the density of states of the sample at the 
position of the tip, $\rho_{s}\left(\vec{r}_{t}, E\right)$ :

$$
I\left(\vec{r}_{t}, V\right)=\frac{2 \pi e}{\hbar} \int_{E_{F}-e V / 2}^{E_{F}+e V / 2} \rho_{t}(E-e V / 2) \rho_{s}\left(\vec{r}_{t}, E+e V / 2\right) d E
$$

Here the electronic density of states of the sample at the position of the tip is defined as

$$
\rho_{s}\left(E, \vec{r}_{t}\right) \equiv \sum_{n}\left|\psi_{n}\left(\vec{r}_{t}\right)\right|^{2} \delta\left(E-\varepsilon_{n}\right)
$$

where $\varepsilon_{n}$ is the energy of the state $\psi_{n}$.

\subsubsection{The STS approximation of Lang}

Lang applies the theory of Tersoff and Hamann to a situation where the LDOS is known at both surfaces (comprised of single atoms) but not in the vacuum [71]. By

allowing the wavefunctions to decay with a simple WKB form [75], Lang estimates the LDOS in the vacuum, $\rho(\vec{r}, E)$, in terms of the LDOS at the surface atom, $\rho(E)$, as

$$
\rho(\vec{r}, E) \approx \rho(E) \exp \left\{-2\left|\vec{r}-\vec{r}_{0}\right| \sqrt{2 m / \hbar^{2}\left(E_{F}+\phi-E\right)}\right\}
$$

Here $\vec{r}$ is some position in the vacuum, $\vec{r}_{0}$ is the position of the surface atom, $\left|\vec{r}-\vec{r}_{0}\right|$ is the distance into the vacuum (i.e. the width of the tunneling barrier), $m$ is the mass of the electron, and $\phi$ is the work function.

He et al. extends this method to include spin and non-zero temperature [76], resulting in an expression for the spin-polarized current,

$$
I^{\uparrow(\downarrow)}\left(\vec{r}_{t}, V\right)=\frac{2 \pi e}{\hbar} \int_{-\infty}^{+\infty} \rho_{t}^{\uparrow(\downarrow)}(E-e V / 2) \rho_{s}^{\uparrow(\downarrow)}(\vec{r}, E+e V / 2) F(E) d E
$$

where $\rho_{s}^{\uparrow(\downarrow)}(\vec{r}, E)$ is the spin-up (spin-down) LDOS as described by eq. 5.3, and $F(E)$ 
is a product of Fermi functions,

$$
\begin{aligned}
F(E) \equiv & f(E-e V / 2)(1-f(E+e V / 2))- \\
& (1-f(E-e V / 2)) f(E+e V / 2)
\end{aligned}
$$

\subsubsection{Superposition of atomic charge-density}

The method of the superposition of atomic charge-densities [77] was successfully used to confirm the structure of the reconstructed $\mathrm{Si}(111)-(7 \times 7)$ surface in 1986 [69]. This method approximates the LDOS in the vacuum as a sum of spherically symmetric atomic charge-densities,

$$
\rho(\vec{r}) \approx \sum_{i} \phi\left(\left|\vec{r}-\vec{r}_{i}\right|\right)
$$

where $\vec{r}_{i}$ is the position of atom $i$ and $\phi(r)$ is an analytic approximation for the charge density far from the nucleus of an isolated atom. The calculation of constant-current images proceeds in the same manner as the Tersoff-Hamann method, by calculating isosurfaces of constant LDOS.

\subsubsection{SLSTM}

We introduce a newly developed method which we have implemented in a Fortran package we call the Simple Lang STM Simulator, or SLSTM. In our method we calculate the LDOS in the vacuum in a manner analogous to the superposition of atomic charge-density method; however, in place of charge density $\phi(r)$, we instead 
use Lang's expression for LDOS in the vacuum (eq. 5.3), resulting in

$$
\rho_{s}(\vec{r} ; E) \approx \sum_{i} \rho_{i}(E) \exp \left\{-2\left|\vec{r}-\vec{r}_{i}\right| \sqrt{2 m / \hbar^{2}\left(E_{F}+\phi-E\right)}\right\}
$$

This expression for the sample LDOS is then used in the tunneling current equation of He et al., eq. 5.4, to calculate the tunneling current for a tip at an arbitrary position in the vacuum and at an arbitrary bias, $I(\vec{r} ; V)$. Once we know this, it is a simple matter to calculate our quantities of interest as they are simple functions of $I(\vec{r} ; V)$.

\subsection{Application to $\mathrm{Si}(111)-(7 \times 7)$}

Confirming the geometry of the reconstructed $\mathrm{Si}(111)-(7 \times 7)$ surface is a classic triumph of the scanning tunneling microscope [78]. Here we use SLSTM on a simple model of the $\mathrm{Si}(111)-(7 \times 7)$ reconstructed surface and make comparisons to previous computational and experimental results.

\subsubsection{Model}

The surface of our system consists of the reconstructed bilayer with twelve adatoms as described by the dimer-adatom-stacking fault model [79] (figure 5.1, from [80]), with periodic boundary conditions. The substrate is represented by a single unreconstructed bilayer, fully passivated below with hydrogen. The hydrogen atoms and the bottom half of this bilayer were fixed while the rest of the system was optimized.

The optimization and electronic structure calculations were performed in the frame-

work of density functional theory using the VASP [81-83] code with PAW potentials 

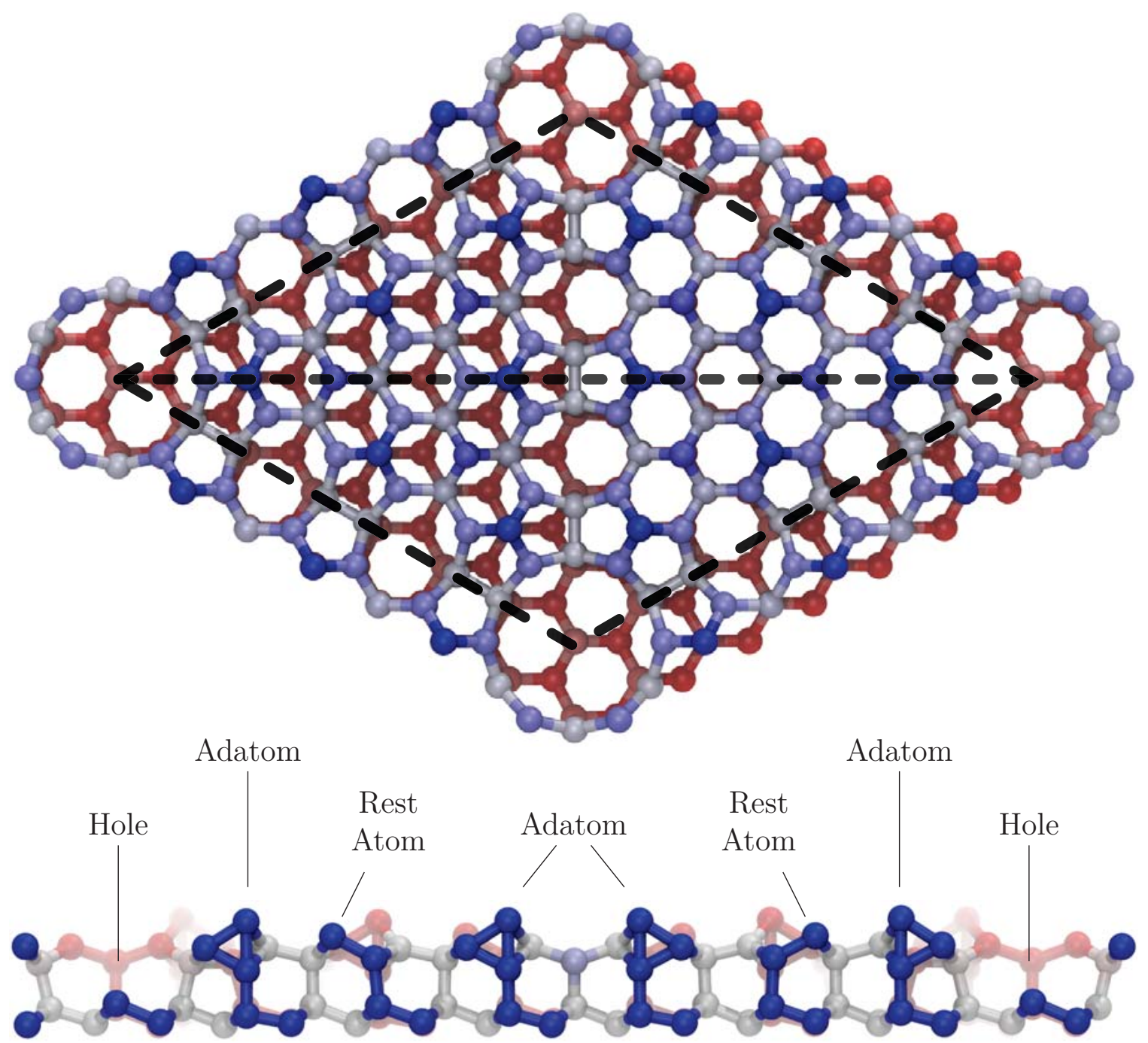

Figure 5.1: The Dimer-Adatom-Stacking Fault model of the $\operatorname{Si}(111)-(7 \times 7)$ reconstructed surface was first proposed by Takayanagi [79]. In both the top-down view (top panel) and the cross section side view (bottom panel), the blue colors indicate atoms that are closer to the viewer, while red indicates atoms that are further away. The unit cell is indicated by the dashed parallelogram. The line scans run from one 'corner atom' to another along the long diagonal, indicated by horizontal dashed line, which is the same path as the cross section. Here faulted half is shown on the right, and the unfaulted half is on the left. 
[84] and PBE functional [85]. The ground structure was obtained by relaxing the structure until forces were less than $0.03 \mathrm{eV} / \AA$.

Two different models were used for the tip. One tip was modeled with a 25-atom Si cluster cut from bulk, with all except the apex atom passivated with hydrogen, and with the apex atom relaxed. Another tip consisted of a pyramid of $11 \mathrm{~W}$ atoms cut from bulk with no optimization. It is commonly seen in experiments that intentionally crashing a clean metal tip into the silicon surface improves the resolution of constant current images, and so the Si-tip model is intended to mimic a contaminated tip while the $\mathrm{W}$ tip is intended to model a pristine tip.

\subsubsection{Results}

We calculated constant current (CCI) images and current imaging tunneling spectroscopy (CITS) images using SLSTM. Figure 5.2 shows a comparison between CCI images at positive and negative bias a,d) from experiment [72], b,e) from previous calculations [72], c,f) from SLSTM, and g) a scanning force microscopy (SFM) image from experiment [87].

Figure 5.3 shows CITS images at negative voltages a) from experiment [72], b) from previous calculations [72], and c) from SLSTM.

From the CCI images we calculated line scans across the long diagonal of the unit cell, which corresponds to the cross section in figure 5.1 (lower panel). This gives us surface height as a function of distance, and allows us to perform quantitative comparisons between our simulations and experimental results published by five different research groups. 


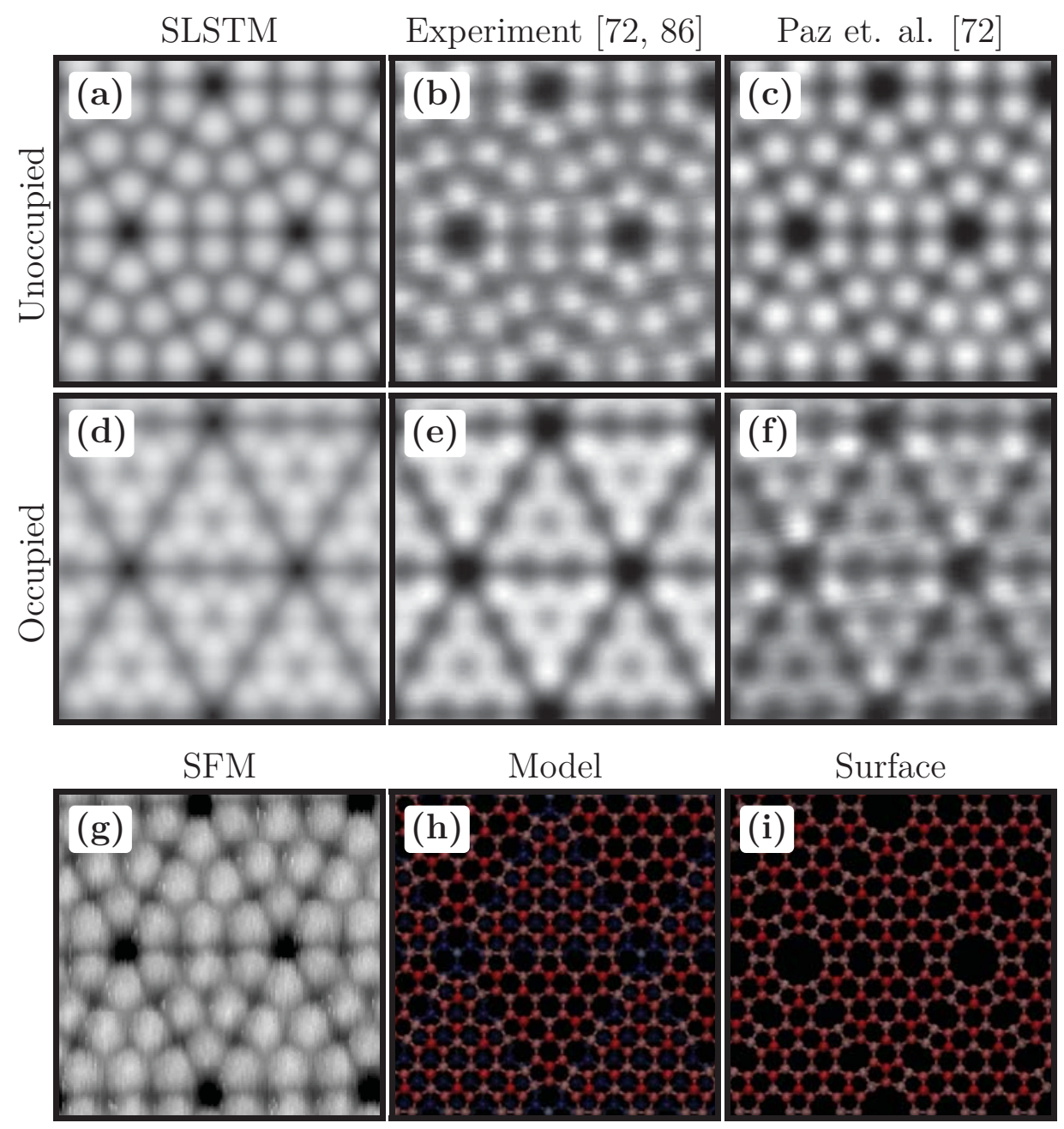

Figure 5.2: Constant current images for the $\mathrm{Si}(111)-7 \times 7$ surface (first two rows). Results from SLSTM (first column) are compared to experiment [72, 86] (middle column) and previous calculations [72] (last column). Set current is $0.2 \mathrm{nA}$. Sample bias is positive for the top row $(+1.5,+1.5$, and $+1.4 \mathrm{~V})$, probing the unoccupied states, and negative for the middle row $(-1.3,-1.5$, and $-1.5 \mathrm{~V})$, probing the occupied states. The topography of the surface without electronic effects can be seen with $(\mathrm{g})$ scanning force microscopy [87]. The atomic positions underlying the simulations is shown in (h), where the Si atoms are colored according to z-height, ranging from blue (lowest) to red (highest). For clarity, the model is shown in (i) without the substrate.

In the simulation results, the center of the line scan data $(x=0)$ is midway between the central adatoms, and corresponds closely to a local minimum of the line scan height. For each experimental data set the precise locations of the atoms in not known, 


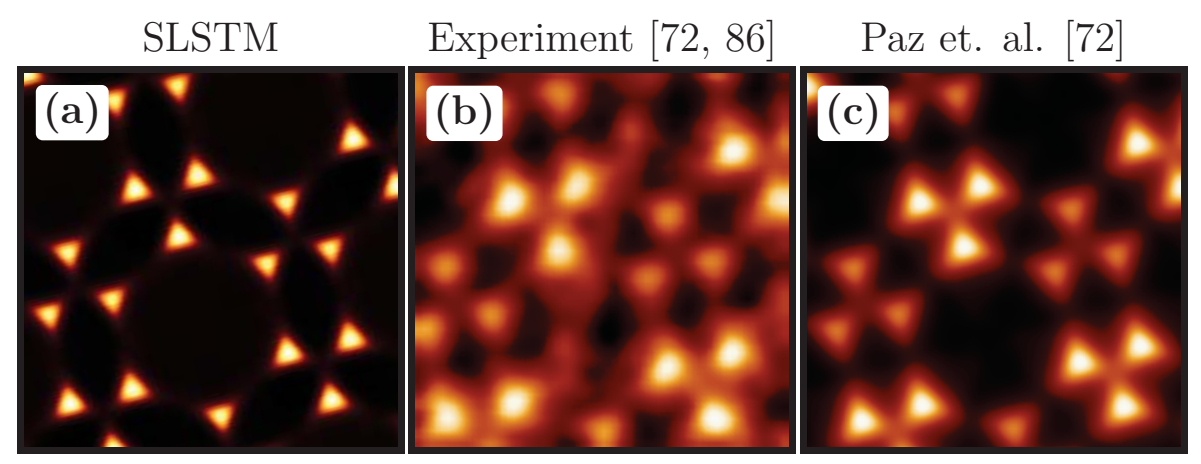

Figure 5.3: Current imaging tunneling spectroscopy (CITS) images for the $\mathrm{Si}(111)-(7 \times 7)$ surface based on the constant current topology at +1.75 $\mathrm{V}$ bias and $2.0 \mathrm{nA}$ set current. CITS bias is $-0.65,-0.85$, and $-0.64 \mathrm{~V}$ for SLSTM, experiment $[72,86]$, and previous calculations [72], respectively.

so $x=0$ is set at the center of the central minimum. From the simulation data, we see that this approximation can be expected to introduce a misalignment of up to -0.3 to $+0.1 \AA$, depending on the bias voltage. However, the differences between the location of the geometric center and the central minimum are a results of asymmetry between the central adatoms, and for each of these data sets this asymmetry is minimal. Each data set is then truncated to the range -18.4 to $+18.4 \AA$. The unfaulted half of the unit cell is in the $y<0$ side and the faulted half is on the $y>0$ side. Since the absolute heights of the line scans are not known for the experimental data sets, $y=0$ is also set at the bottom of the central minimum. Before comparing two line scans, the data are aligned by adding a vertical offset, chosen through least-squares fitting.

In the superposition method as implemented by Tromp [69], there is no electronic contribution to the line scan shape, and the shape is determined entirely by the geometry of the system. Tromp found excellent agreement between the superposition method line scans and experimental data taken at $+2.0 \mathrm{~V}$, so we use Tromp's $+2.0 \mathrm{~V}$ experimental data as a proxy for what the height profile would be based solely on geometry. Figure 5.4 shows the root-mean-square-deviation between Tromp's data and simulations performed over a wide range of voltages and set currents, using the Si tip. 


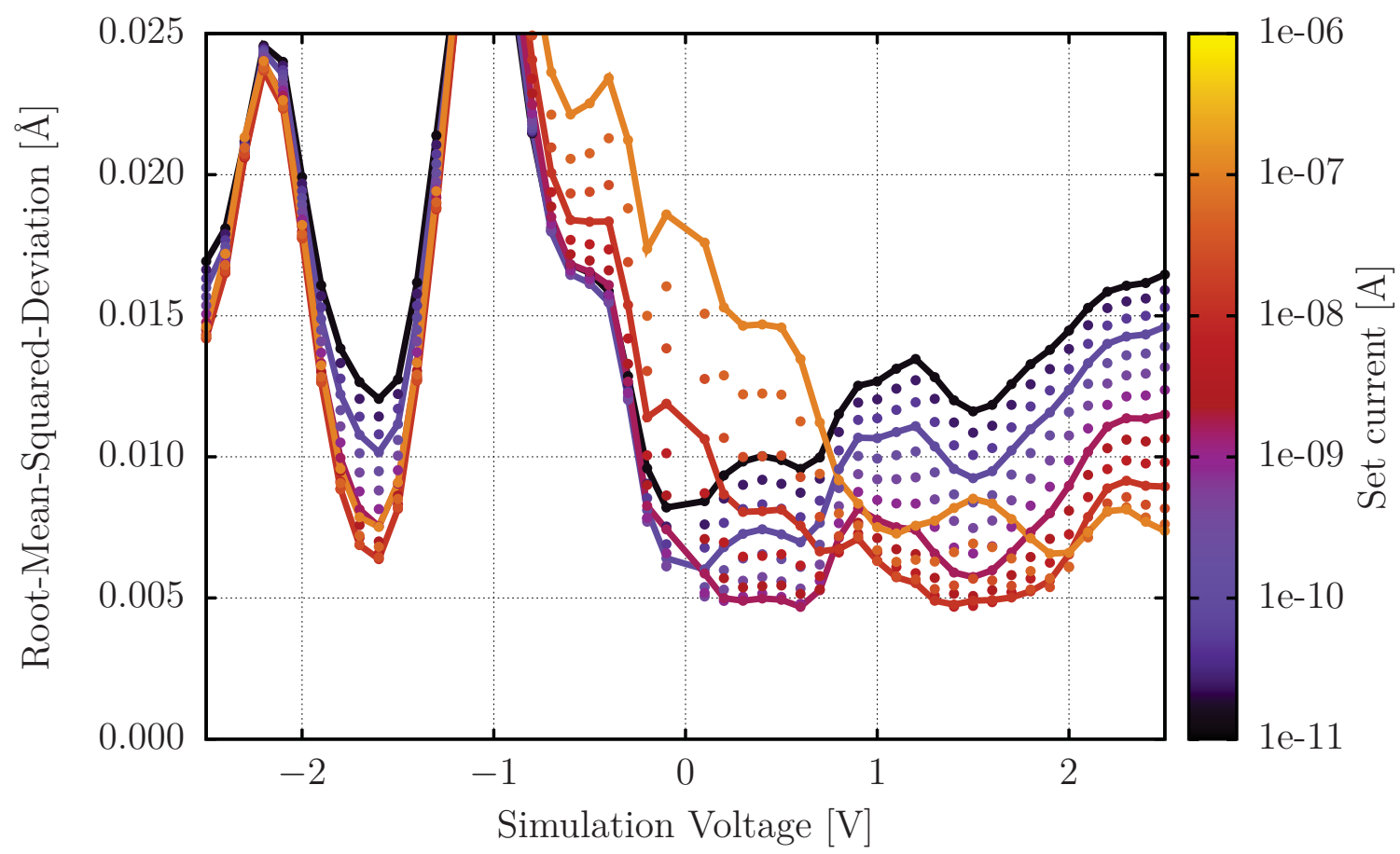

Figure 5.4: The experimental linescan measured by Tromp et al. at +2 $\mathrm{V}$ are used as an approximation to the linescan that would be produced based solely on the geometry of the system. We calculated linescans using SLSTM over a range of voltages and set currents, and determined how much the calculated linescans deviate from a linescan determined solely by the geometry, using a measure of the badness of the fit between the two lines. In this figure, each point represents a single calculated linescan. The x-axis shows the bias voltage used in the calculation, and the color indicates the set current. The badness of fit is plotted on the y-axis, with values less than $\sim 0.007 \AA$ representing particularly good fits, and values over $\sim 0.01 \AA$ representing rather poor fits.

To complement figure 5.4, figure 5.5 shows simulated line scans at voltages +1.5 , $+0.5,-1.0$, and $-1.6 \mathrm{~V}$, and at a set current of $1.6 \mathrm{nA}$. The offset for each line scan is chosen by fitting to the experimental data (dashed line).

Figure 5.6 shows the projected density of states for the two distinct adatoms (at $-18 \AA$ and at $-3 \AA$ ) and the rest atom (at $-13 \AA$ ) in the unfaulted half of the unit cell, as well as the density of states for the Si and the W tips. 


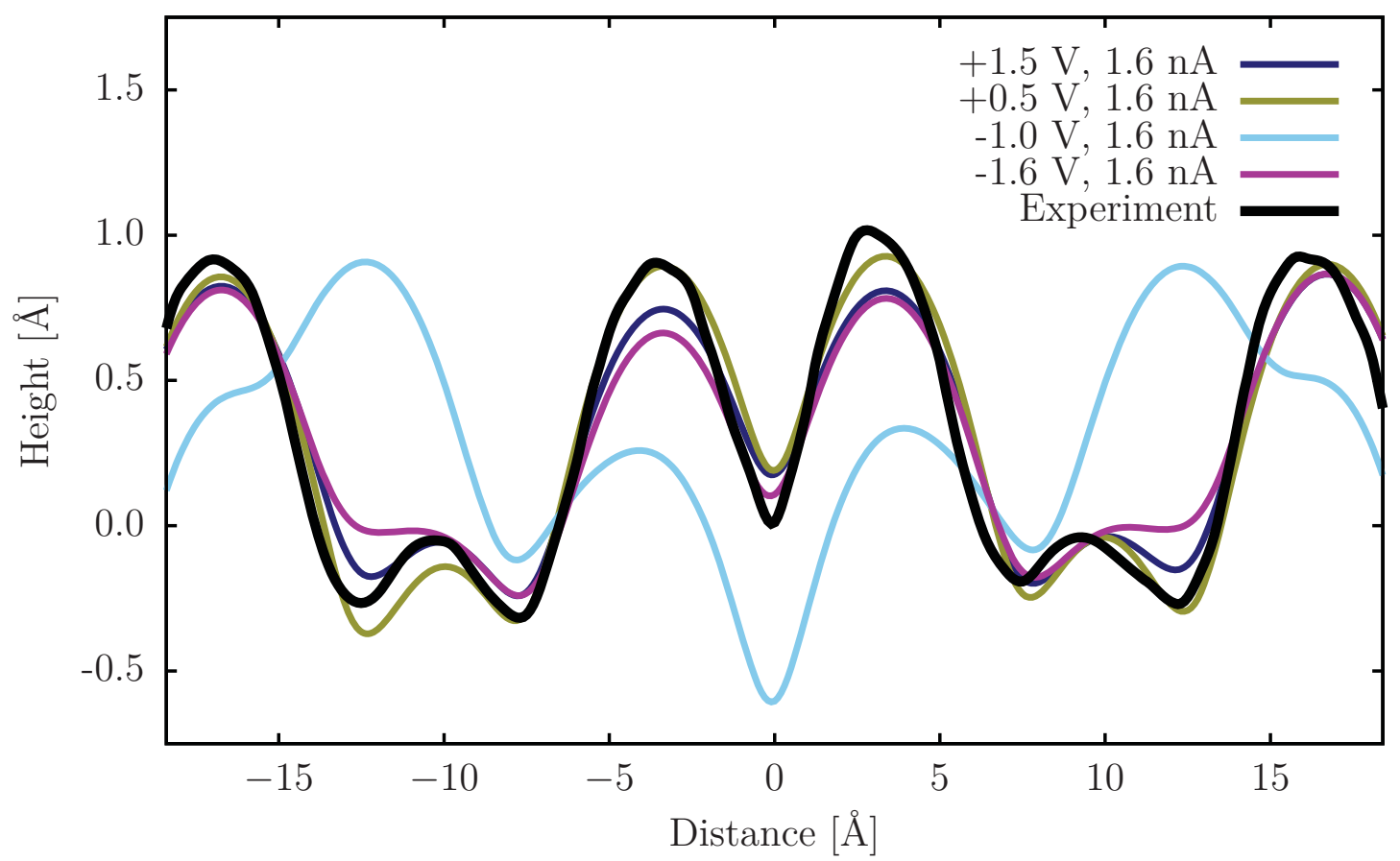

Figure 5.5: Line scans across the long diagonal at positive voltages (purple and green lines) show quantitative agreement with experiment [69] (dashed line). For negative voltages the rest atom (located at $\pm 13 \AA$ ) contributes more than the adatoms, creating a pattern distinct from the positive voltage results as exemplified at $-1.0 \mathrm{~V}$ (blue line). Surprisingly, good agreement can also be found over a very narrow range of negative voltages (pink line).

\subsubsection{Discussion}

From figure 5.2 we can see that at $+1.5 \mathrm{~V}$ SLSTM produces CCI images that are in agreement with experiment and previous calculations. Interestingly, even closer agreement is seen between the SLSTM CCI image and the experimental SFM image. This suggests that at this voltage SLSTM produces an image that is dominated by the geometry of the system. This idea is corroborated by the results of figure 5.4, where we can see that at $+1.5 \mathrm{~V}$ and $0.2 \mathrm{nA}$ the fit between the SLSTM results and the geometric linescan is about $0.007 \AA$, which is a moderately good fit. 


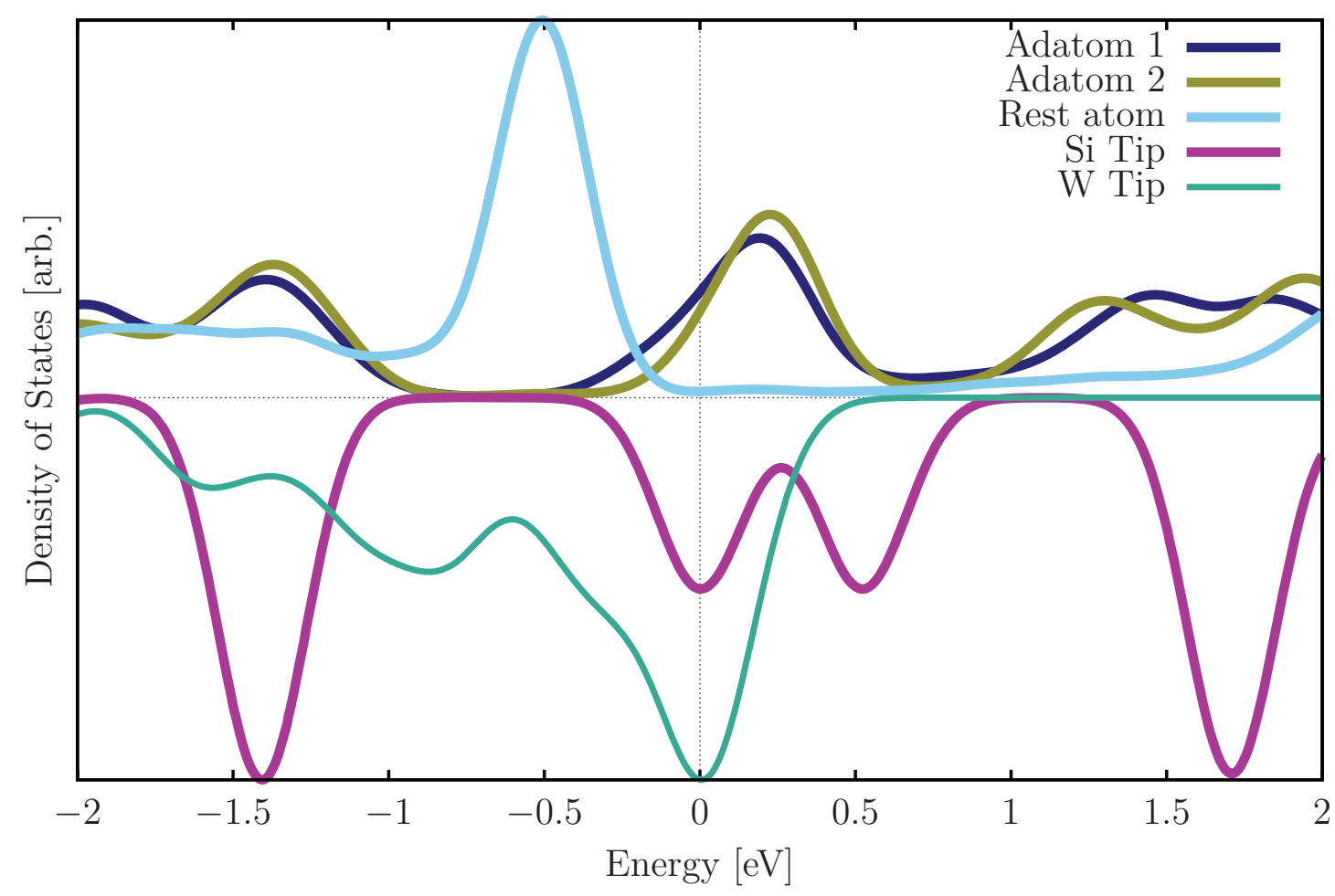

Figure 5.6: Atom-projected density of states for the $\operatorname{Si}(111)-(7 \times 7)$ reconstructed surface are shown for the two unique adatoms and one unique rest atom in the unfaulted half of the unit cell. The adatoms are very similar to each other and quite distinct from the rest atom. Also shown is the total DOS for the Si tip and the W tip.

For a range of negative voltages the rest atoms (see fig. 5.1) are visible and even appear to be as tall as the adatoms, clearly differentiating the image from the geometric image. This can be explained by looking at the projected density of states (PDOS) in figure 5.6, where it can be seen that for a range of energies from about -1.0 to about $-0.3 \mathrm{eV}$ the DOS is dominated by the rest atoms.

A subtle but notable feature that this simulation fails to reproduce is the difference in apparent height between the faulted and unfaulted regions. The PDOS at the surface of our model is essentially the same in the faulted as in the unfaulted regions, so our method and the method of Paz et al. would both be expected to fail to reproduce this 
difference in height given our model as input. From this we conclude this difference can be attributed to the slight differences in the input models.

In figure 5.3 we see qualitative agreement between both computational methods and the experimental CITS image. The dominant feature is the trio of triangular bright spots above the rest atoms, with a difference in brightness between the faulted and unfaulted regions. This is reproduced in both simulations, but our simulation seems again to understate the faulted/unfaulted difference. Neither simulation seems to adequately describe the hole region.

In figure 5.4 we see that for positive voltages probing unoccupied states and moderate set currents the linescans produced by SLSTM follow very closely the geometry of the system, with little apparent influence from the electronic structure of the surface. In contrast we see that over a range of approximately $-0.3 \mathrm{~V}$ to $-1.4 \mathrm{~V}$ and for voltages less than about $-1.8 \mathrm{~V}$ the linescans differ strongly from that expected purely due to geometry. It is over this range that the rest atoms become visible and the standard 12-atom image gives way to the 18-atom image. We can see this in the linescans in figure 5.5, where we have selected voltages that correspond to interesting features in figure 5.4. At positive voltages and at $-1.6 \mathrm{~V}$, we see the linescans follow closely the geometry. However at $-1.0 \mathrm{~V}$ we see strong disagreement, as the rest atom dominates the linescan.

This result can be explained by comparing the projected density of states (PDOS) of the surface atoms and the density of states (DOS) of the tip (figure 5.6). For positive voltages, electrons in the occupied states in the tip tunnel to the unoccupied states in the surface, and these unoccupied states are primarily associated with the adatoms. Since the adatoms are the 'tallest' surface atoms, an image that is dominated by the adatoms will be similar to the geometrical image. For negative voltages, it is the occupied surface states that are involved in conduction, and for a narrow energy 
range these states are overwhelmingly associated with the rest atoms. This allows the rest atoms to appear taller than the adatoms, as seen in figure 5.5. Interestingly, at a bias of $-1.6 \mathrm{~V}$ the PDOS peak associated with the rest atoms (at $-0.5 \mathrm{eV}$ ) lines up with a gap in the DOS of the tip $($ at $+1.1 \mathrm{eV})$, severely reducing the rest atom contribution and restoring the "geometric" image.

\subsection{Application to $(\mathrm{PbS})_{32}$}

We recently investigated the electron transport properties of the $(\mathrm{PbS})_{32}$ "baby crystal" quantum dot on a gold substrate using the SLSTM method and code (Electron tunneling characteristics of a cubic quantum dot, $(\mathrm{PbS})_{32}$. SANJEEV K. Gupta, Haiying He, Douglas Banyai, Anil K. Kandalam, and Ravindra PAndey. The Journal of Chemical Physics, 2013.) [4]. We found that despite the significant band gap $(\sim 2 \mathrm{eV})$ of the isolated quantum dot, the interaction between the dot and substrate creates states near the Fermi level that facilitate a measurable tunneling current. This effect is found to be sensitive to the substrate, in that differences are found between $(\mathrm{PbS})_{32}$ quantum dots on $\mathrm{Au}(110)$ versus $\mathrm{Au}(001)$ surfaces. This section contains a summary of the above referenced work, with some details relegated to the associated journal article.

\subsubsection{Introduction}

Lead-chalcogenide semiconductors are actively being explored for a variety of applications. For example, $\mathrm{PbS}$ nanowires were recently used to build field-effect transistors [88]. PbS semiconducting quantum dots hold promise for applications in photovoltaics and infrared sensors (e.g. [89]), as demonstrated in a recent study that explored the 


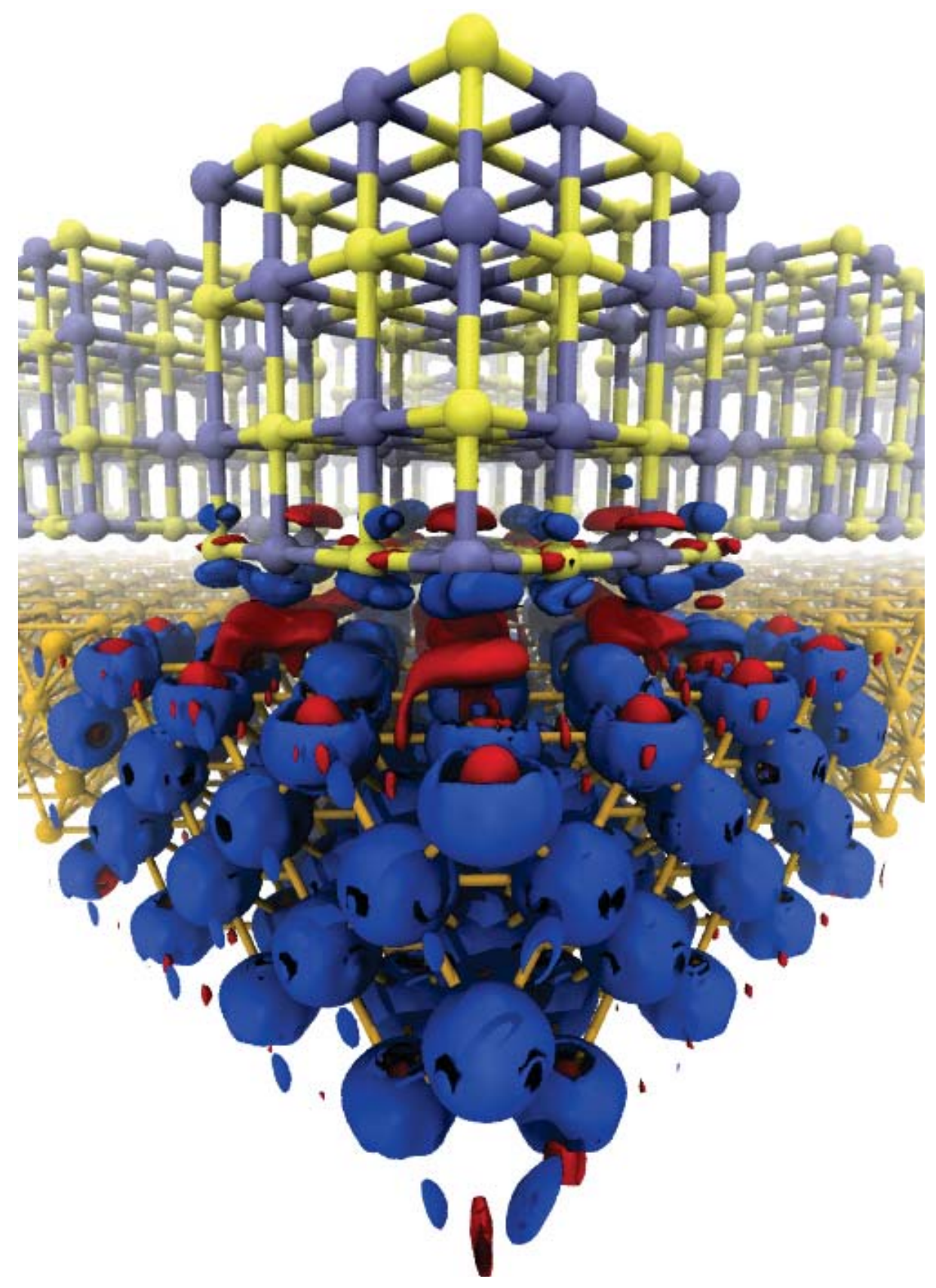

Figure 5.7: The $(\mathrm{PbS})_{32}$ "baby crystal" is shown on a $\mathrm{Au}$ surface. The transfer of charge between the substrate and the QD is illustrated with isosurfaces of charge density difference. Blue indicates regions of electron accumulation, and red are regions of electron depletion. 
performance of $\mathrm{PbS}$ quantum dot photovoltaics as a function of electronic band gap via controlling the size of the quantum dots [90].

$\mathrm{PbS}$ forms crystalline quantum dots as small as $(\mathrm{PbS})_{32}$, which due to its bulklike crystal structure is sometimes called a "baby crystal" [91]. Many studies have looked at small $\mathrm{PbS}$ quantum dots in the range of 2-10 nm, but to date, few studies have looked at the electronic transport properties of ultrasmall $(<1 \mathrm{~nm})$ quantum dots. However, in recent experiments such quantum dots were synthesized and then imaged under a scanning tunneling microscope (STM) [91]. In this work we calculated electronic transport properties of $(\mathrm{PbS})_{32}$ quantum dots on both $\mathrm{Au}(110)$ and $\mathrm{Au}(001)$ surfaces in an STM-like configuration (figure 5.7).

\subsubsection{Model}

The ground state geometry of the cubic, 64-atom $(\mathrm{PbS})_{32}$ quantum dot (QD) was calculated at the PW91-DFT level of theory [92] using the Vienna ab initio Simulation Package (VASP) [81-83]. The $\mathrm{Au}(001)$ surface was represented by a slab with four layers and 32 atoms per layer, resulting in a $16.7 \AA \times 16.7 \AA$ unit cell with periodic boundary conditions. The $\mathrm{Au}(110)$ surface was represented by a slab with five layers and 24 atoms per layer, resulting in a $16.7 \AA \times 17.7 \AA$ unit cell. A separation of $2.8 \AA$ was placed between the substrate and the $(\mathrm{PbS})_{32} \mathrm{QD}$. This distance was found to be the equilibrium distance by calculating the total energy of the system as a function of separation distance. $15 \AA$ of vacuum was added in the z-direction to isolate the slab and quantum dot from their periodic images. The $(\mathrm{PbS})_{32}$ quantum dot is about

$9 \AA$ wide, leaving a minimum separation of about $8 \AA$ between images in the lateral directions. 

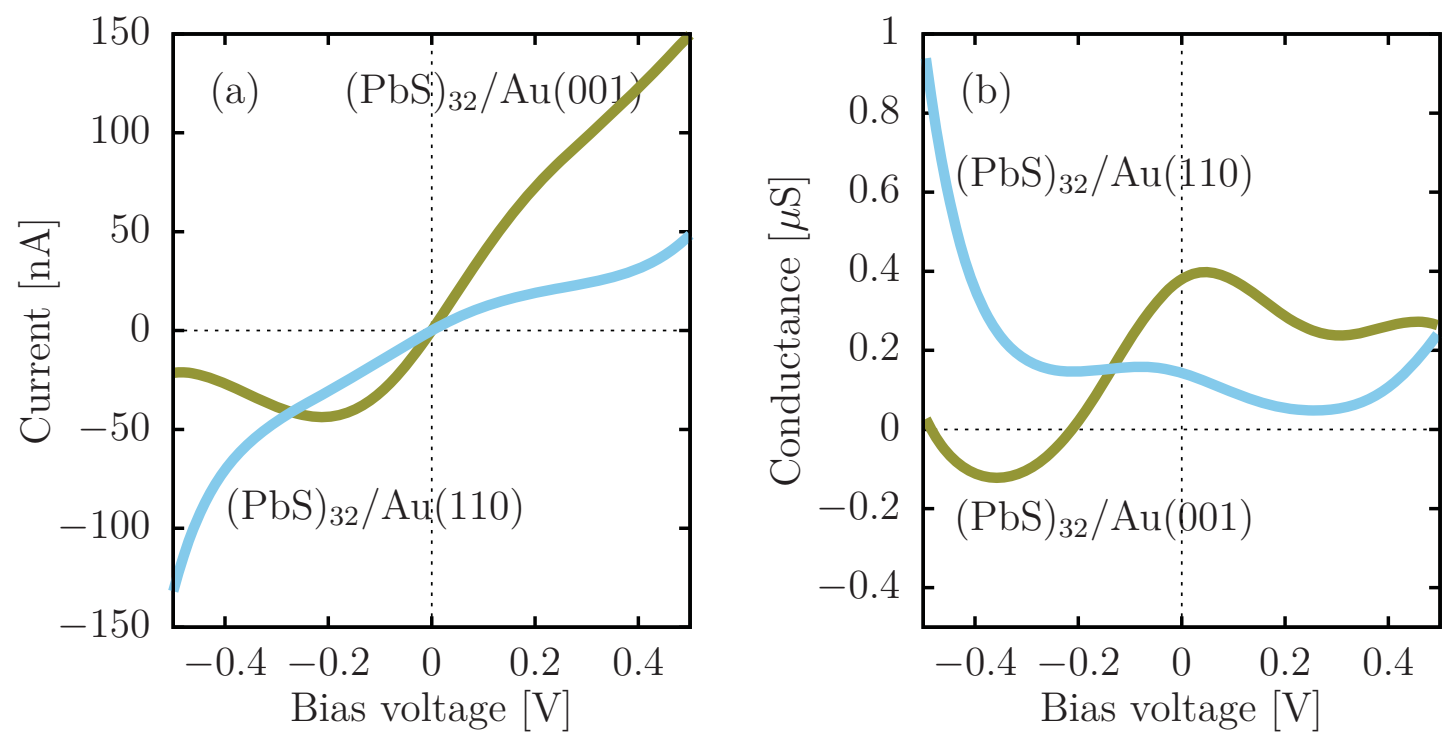

Figure 5.8: (a) The $(\mathrm{PbS})_{32}$ quantum dot shows distinctly different currentvoltage characteristics when on an $\mathrm{Au}(001)$ surface (dashed green line) versus an $\mathrm{Au}(110)$ surface (dotted blue line). (b) The differential conductance graph shows a significant negative differential resistance (NDR) effect, which is strongly dependent upon the $\mathrm{Au}_{13}$ tip we chose.

The cap of the STM tip is represented by an $\mathrm{Au}_{13}$ cluster, which is roughly spherical. For some comparisons, a larger $\mathrm{Au}_{43}$ cluster is used, which was cut from bulk and optimized, leaving it also roughly spherical and with an FCC-like structure. The current-voltage measurements are calculated with the tip at a distance of $5 \AA$ above the $(\mathrm{PbS})_{32} \mathrm{QD}$. A positive bias voltage indicates the substrate is at a positive bias relative to the tip. More specifically, for a bias of $V$, the substrate is biased to $+\frac{1}{2} V$, and the tip is biased to $-\frac{1}{2} V$.

\subsubsection{Results and Discussion}

In panel (a) of figure 5.8, we show currents calculated as a function of bias voltage over the range $\pm 0.5 \mathrm{~V}$. For both systems, the $I-V$ curves are significantly non-ohmic. 

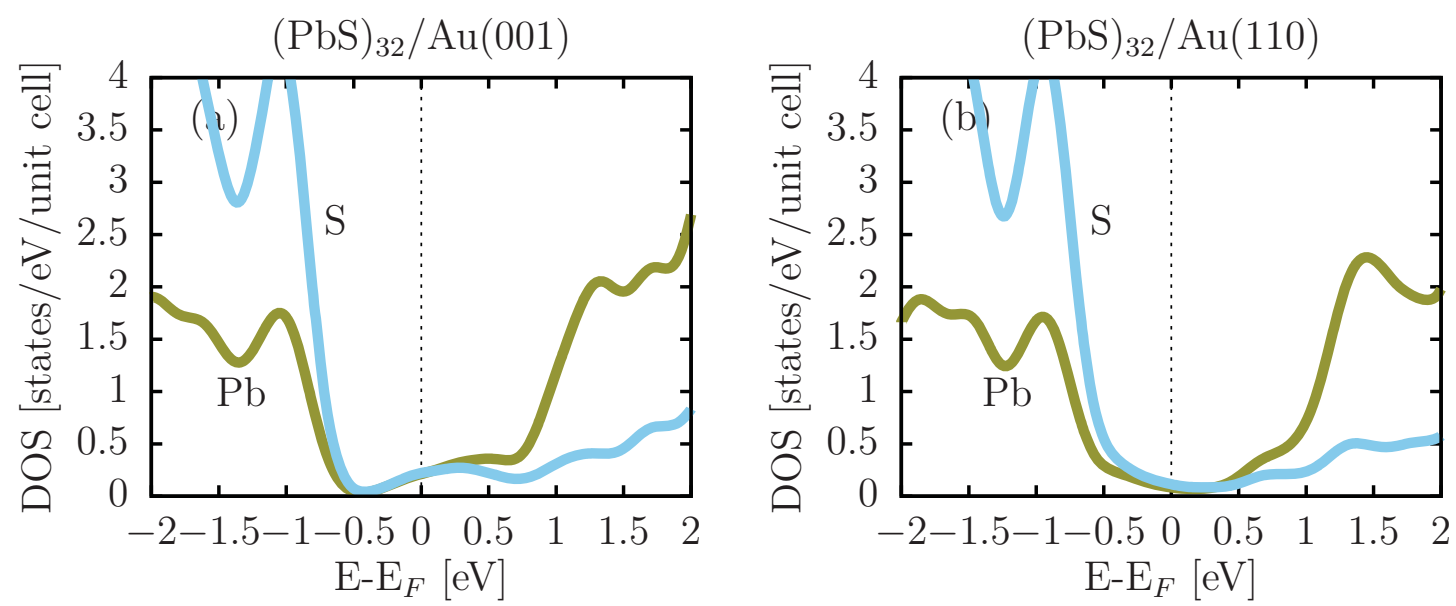

Au13
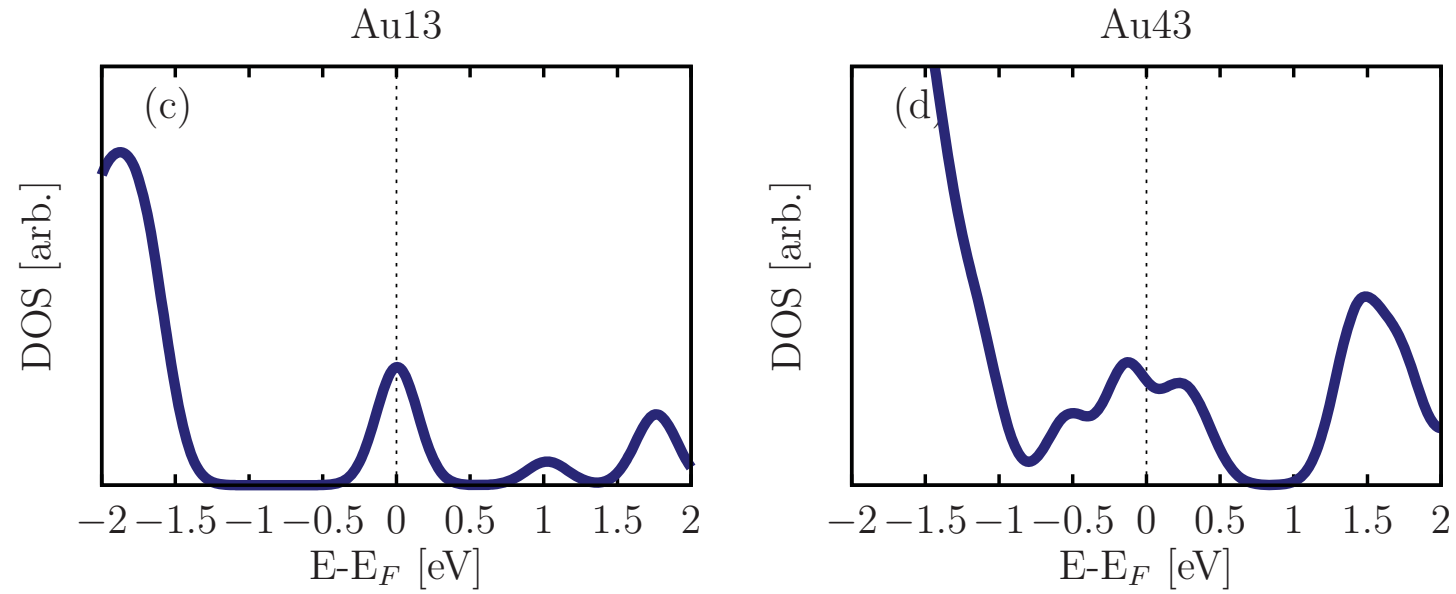

Figure 5.9: The atom projected density of states (PDOS) are calculated for for (a) $(\mathrm{PbS})_{32} / \mathrm{Au}(001)$ and $(\mathrm{b})(\mathrm{PbS})_{32} / \mathrm{Au}(110)$. The PDOS associated with $\mathrm{Pb}$ is indicated with a dashed green line, and that associated with $\mathrm{S}$ is indicated with a dotted blue line. The total density of states (DOS) is shown for the (c) $\mathrm{Au}_{13}$ and (d) $\mathrm{Au}_{43}$ tips.

This is highlighted in the differential conductance curves in panel (b), where it can be seen that the $\mathrm{Au}(001)$ system exhibits negative differential resistance (NDR) of a significant magnitude.

To understand this result, consider the density of states (DOS) for the system and the tip. In figure 5.9 we show the DOS associated with the $\mathrm{Pb}$ and $\mathrm{S}$ atoms in the $\mathrm{Au}(001)$ system along with the total DOS of the tip. Figure 5.10 illustrates the 
overlap between the filled states in the sample and the available states in the tip as the bias voltage is increased. When the bias is zero, there are no available states in the tip to accommodate the filled states in the sample and so there is no current. When the substrate is biased at $-0.3 \mathrm{~V}$, there are now filled states in the sample that are available for tunneling over to the available states in the tip, indicated with crosshatching. An interesting thing happens when we increase the magnitude of the bias from $-0.3 \mathrm{~V}$ to $-0.5 \mathrm{~V}$. Now there are a small number of additional electrons in states deep in the conduction band of the sample that are able to tunnel to the tip, but meanwhile there is a larger number of electrons in states near the Fermi level which now lack corresponding available states in the tip to tunnel to. The net result is that as the bias is increased from $-0.3 \mathrm{~V}$ to $-0.5 \mathrm{~V}$, the total tunneling current decreases, giving rise to negative differential resistance.

Note that this effect is dependent both upon having a minimum of the DOS offset from the Fermi level, and upon the existence of a strongly peaked DOS for the tip. Comparing panels (c) and (d) of figure 5.9, we can see that the $\mathrm{Au}_{43}$ tip does not exhibit such prominent peaks, and thus would not be expected to create a significant NDR. Indeed, in figure 5.11 we can see that the $\mathrm{Au}_{43}$ tip produces only a nominal NDR for the $(\mathrm{PbS})_{32} / \mathrm{Au}(001)$ system. From this we can conclude that the NDR found here is not a general property of the systems we are studying, but rather a very particular result of the interaction between the system and the model we chose to represent the tip. This highlights not only the importance of including the tip when modeling STS properties, but also the possible failings that can occur if the model tip is not representative of the actual tip. 

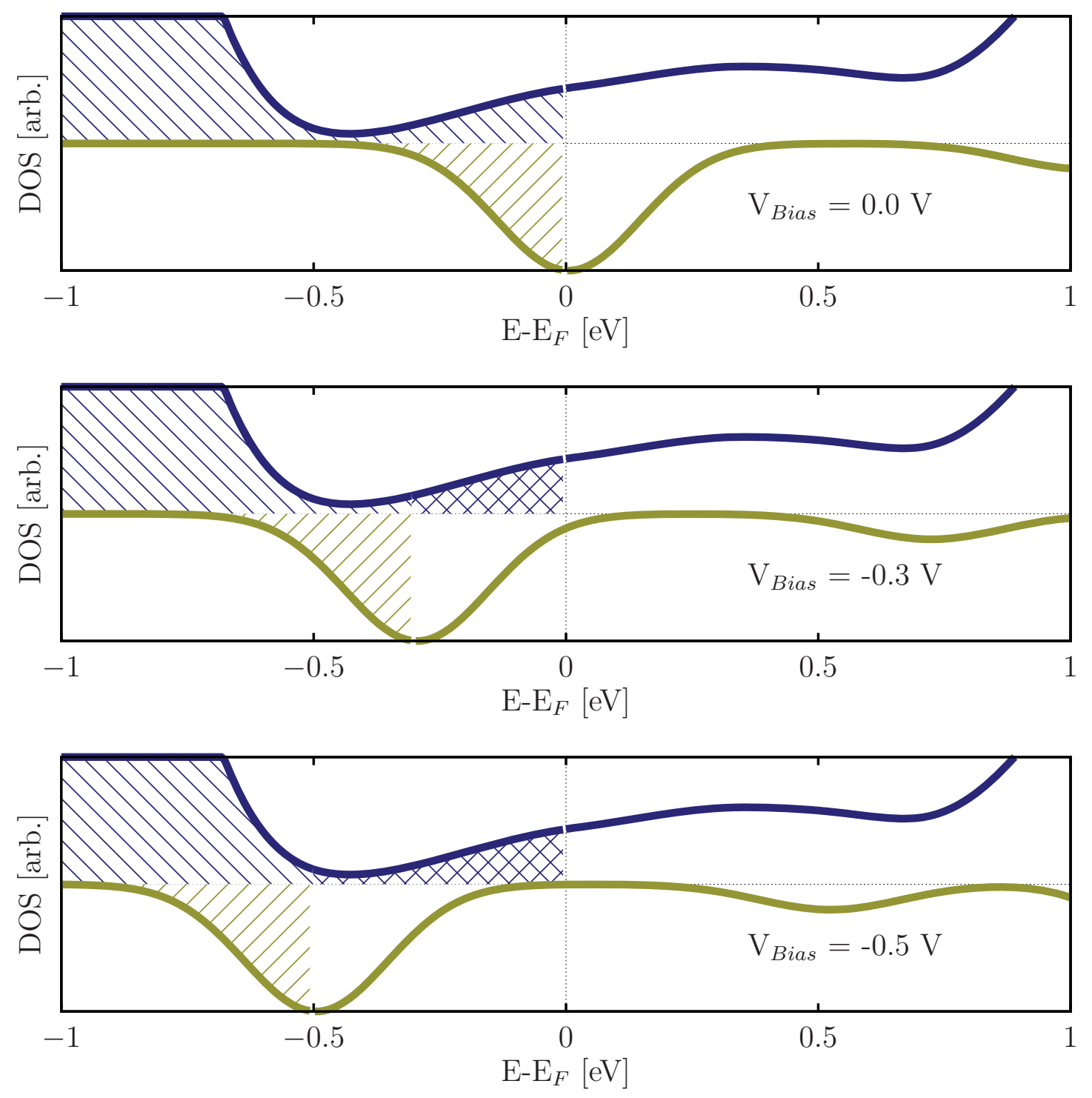

Figure 5.10: The density of states $(\mathrm{DOS})$ of $(\mathrm{PbS})_{32}$ and the $\mathrm{Au}_{13}$ tip are compared as a bias voltage is applied in order to describe the origin of the negative differential resistance (NDR) effect. The $(\mathrm{PbS})_{32}$ total DOS is plotted in purple on the positive $\mathrm{y}$-axis, and the tip DOS is plotted in green on the negative y-axis. The filled regions represent states that are full at zero temperature, and the unfilled regions indicate states that are open. At zero bias (top panel), none of the electrons in the tip or sample see open states to tunnel to, and thus there can be no current. At $\mathrm{V}_{\text {Bias }}=-0.3 \mathrm{~V}$ (middle panel), electrons in the sample with a range of energies (cross-hatched) can tunnel to the tip. However, as the bias is increased to $-0.5 \mathrm{~V}$ (bottom panel), electrons near the Fermi level no longer have a place to go, and there is a net decrease in the number of electrons that have states to tunnel to, despite the increase in energy range. 


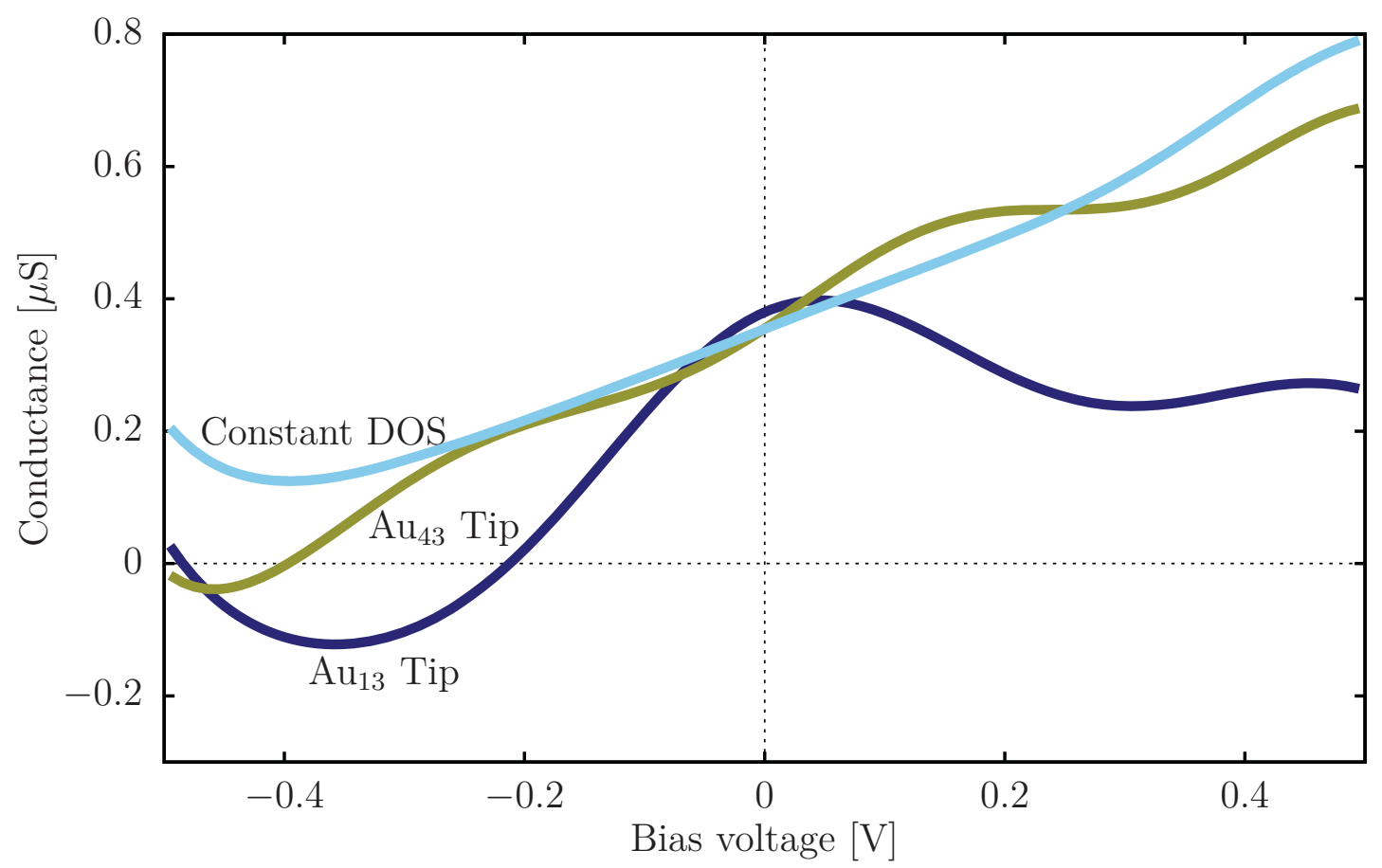

Figure 5.11: Differential conductance characteristics of $(\mathrm{PbS})_{32} / \mathrm{Au}(001)$ are calculated using two alternate tips. In addition to the $\mathrm{Au}_{13}$ tip (purple), a larger $\mathrm{Au}_{43}$ tip (green) is used. For comparison, results are presented for a tip (blue) that has a constant DOS, equal to the value of $\mathrm{Au}_{43}$ tip DOS at $\mathrm{E}=\mathrm{E}_{F}$. The strongly peaked $\mathrm{Au}_{13}$ DOS interacts with the $(\mathrm{PbS})_{32}$ DOS to create a region of NDR. The much smoother $\mathrm{Au}_{43}$ DOS allows only minimal NDR, and the flat DOS of the constant DOS tip creates a strictly non-negative conductance curve.

\subsubsection{Conclusion}

In spite of the significant band gap of the isolated $(\mathrm{PbS})_{32}$ quantum dot, we find significant tunneling currents at relatively low bias. This is attributed to the hybridization of states at the interface between the QD and the underlying surface. The interaction between the $\mathrm{QD}$ and the $\mathrm{Au}(110)$ and $\mathrm{Au}(001)$ substrates are different enough to allow for differentiation based on the differential conductance. 


\subsection{Application to Si-doped BN Monolayer}

This section provides a summary of work we recently published in Nanoscale (Effect of Si doping on the electronic properties of BN monolayer. SANJEEV K. Gupta, Haiying He, Douglas Banyai, Mingsu Si, Ravindra Pandey, and Shashi P. Karna. Nanoscale, 2014.) [5]. Greater detail is provided in the reference and associated supplementary information.

\subsubsection{Introduction}

Much recent work has been done to explore two-dimensional materials for possible use in digital electronics [93], with hexagonal boron nitride monolayers one of the materials of most interest [94], next to graphene. Special attention is given to materials and devices that may be integrated with existing Si-based semiconductor fabrication. Therefore we found it interesting to explore the role of substitutional doping of $\mathrm{Si}$ atoms in BN monolayers. We find that substitutional doping with Si atoms modifies the band structure and thus the electronic properties of the BN monolayer, and that there are noticeable differences depending upon the location of the dopant that should allow for experimental measurements to discriminate between them. 


\subsubsection{Model}

The density-functional-theory-framework was used to calculate the spin-polarized electronic structure of hBN monolayer systems. The exchange and correlation functionals were represented in the generalized gradient approximation (GGA) as described by Perdew, Burke, and Ernzehof [85]. Calculations were performed with the projector augmented wave (PAW) method as implemented in the Vienna ab initio simulation package (VASP) [81-83]. Tunneling properties were calculated with the SLSTM method and code.

The BN monolayers were placed on the top side of an $\mathrm{Au}(111)$ surface at a distance of $3.0 \AA$, a value that was found to minimize the total energy in previous calculations $[95,96]$. The lattices were matched by using a $(4 \times 6)$ supercell for the BN monolayers and a $(6 \times 8)$ supercell for the $\mathrm{Au}(111)$ substrate. The substrate was represented by a slab four layers thick, and $10 \AA$ of vacuum separate the structure from its image in the $\mathrm{z}$-direction.

Three different doping configurations were used for our calculations (figure 5.12), in addition to a pristine un-doped configuration. In the $\mathrm{Si}_{\mathrm{B}}$ configuration, a single $\mathrm{Si}$ atom replaces a single $\mathrm{B}$ atom in the $\mathrm{BN}$ lattice. The $\mathrm{Si}_{\mathrm{N}}$ configuration sees a $\mathrm{N}$ atom replaced by a $\mathrm{Si}$ atom. In the $\mathrm{Si}_{\mathrm{BN}}$ configuration, a single $\mathrm{Si}$ atom replaces a neighboring pair of $\mathrm{B}$ and $\mathrm{N}$ atoms. This results in four-fold bonding between the $\mathrm{Si}$ and the four neighboring $\mathrm{B}$ and $\mathrm{N}$ atoms, creating a so-called "four-fold configuration," in comparison to the "three-fold configurations" of $\mathrm{Si}_{\mathrm{B}}$ and $\mathrm{Si}_{\mathrm{N}}$. In the four-fold configuration, the optimized geometry is planar, while in both of the three-fold configurations the Si atom protrudes from the monolayer as if it were too big for the hole left behind by the vacant $\mathrm{B}$ or $\mathrm{N}$ atom. 


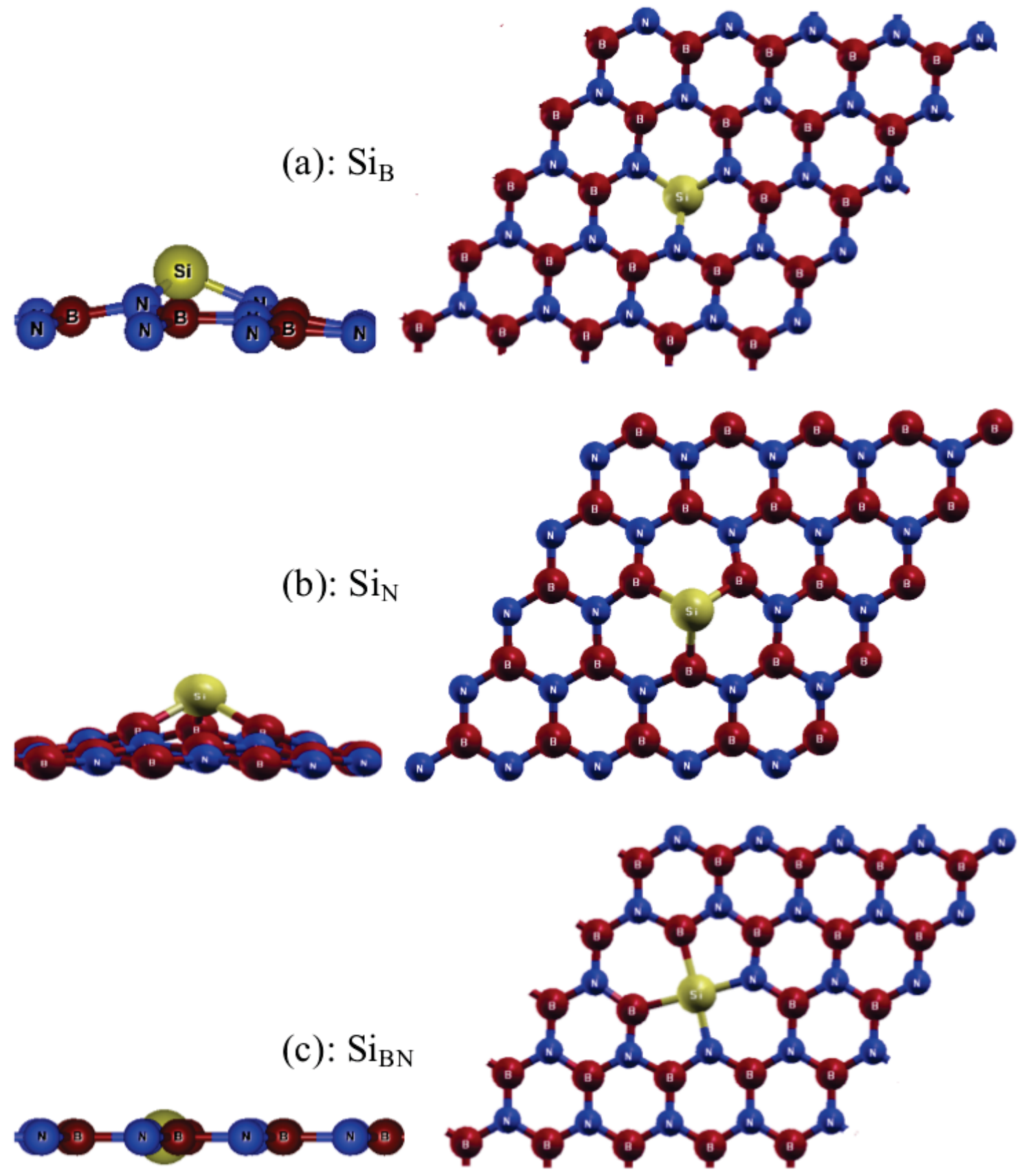

Figure 5.12: The structure of the three-fold coordinated $\mathrm{Si}_{\mathrm{B}}$ (a) and $\mathrm{Si}_{\mathrm{N}}$ (b), and the four-fold coordinated $\mathrm{Si}_{\mathrm{BN}}$ (c) are shown. The top-down views (right side) show the coordination, while the side views (left side) illustrate the significant buckling found in the three-fold coordinated cases. From Gupta, 2014 [5]. Reproduced by permission of The Royal Society of Chemistry. See appendix A for documentation of permission to republish this material. 

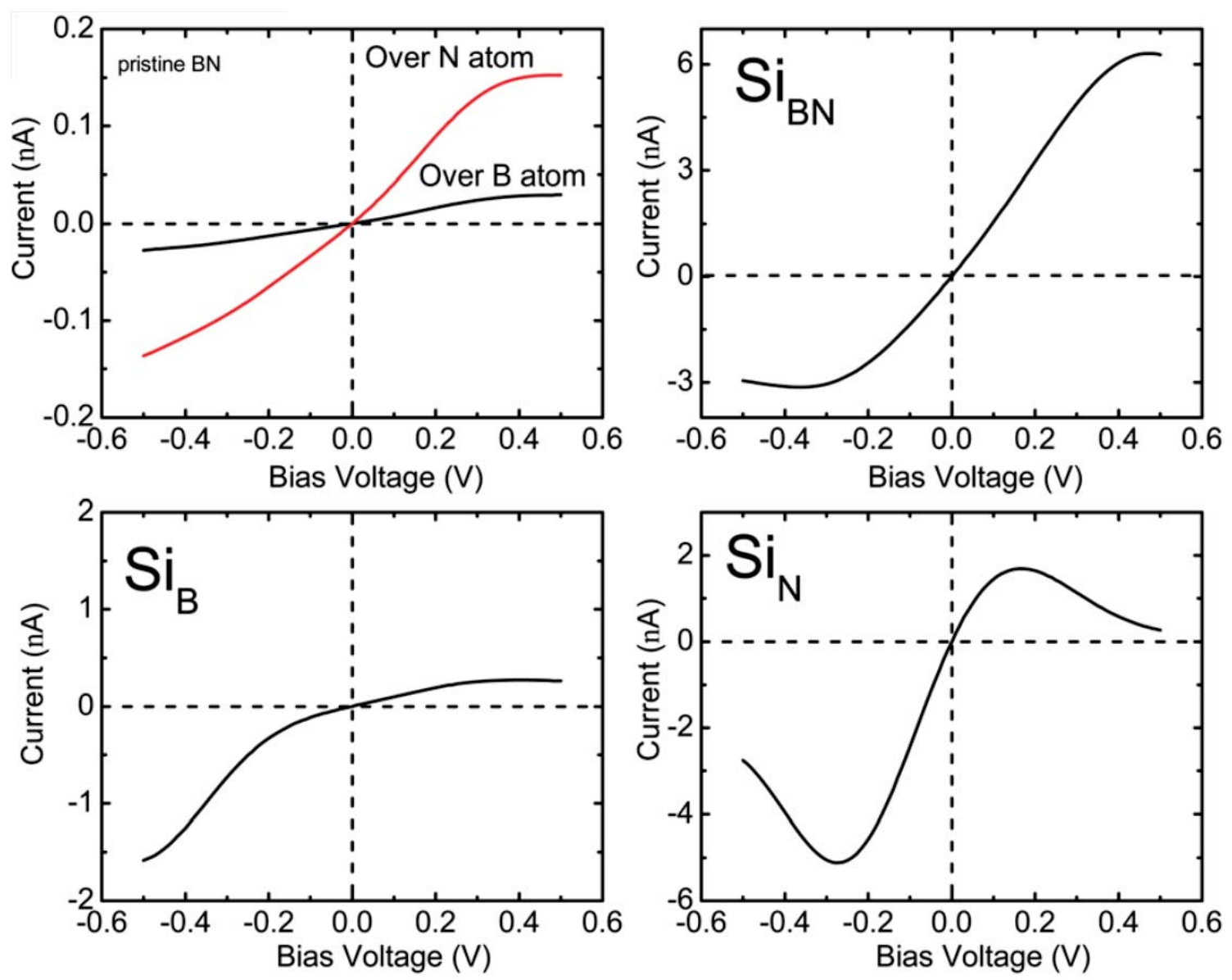

Figure 5.13: In the first panel, current is calculated with a $\mathrm{Au}_{13}$ tip at $+5 \AA$ above a B or $\mathrm{N}$ atom for the pristine BN monolayer (black or red, respectively). The next three panels show the current calculated with the tip $+5 \AA$ above the $\mathrm{Si}$ dopant in the $\mathrm{Si}_{\mathrm{BN}}, \mathrm{Si}_{\mathrm{B}}$, and $\mathrm{Si}_{\mathrm{N}}$ systems. The $I-V$ curve for each is unique, potentially allowing for identification of dopant sites through the scanning tunneling spectroscopy (STS) method. From Gupta, 2014 [5]. Reproduced by permission of The Royal Society of Chemistry. See appendix A for documentation of permission to republish this material.

As in the $(\mathrm{PbS})_{32}$ study above, the tip is modeled with an $\mathrm{Au}_{13}$ cluster. 


\subsubsection{Results and Discussion}

We start by placing the $\mathrm{Au}_{13}$ STM tip $5 \AA$ away from the pristine $\mathrm{hBN}$ monolayer, directly above either a $\mathrm{B}$ or a $\mathrm{N}$ atom. Over a range of $\pm 0.5 \mathrm{~V}$, we find currents on the order of $10^{-10}$ A (panel (a) in figure 5.13). A minimal tunneling current is expected, due to the sizable $4.4 \mathrm{eV}$ band gap of the pristine monolayer.

Next we place the tip above the Si dopant in each of the configurations, keeping a constant $5 \AA$ between the tip and Si Atom (panels (b)-(d) in figure 5.13). Here we find currents 1-2 orders of magnitude larger. Of more interest, while the $\mathrm{Si}_{\mathrm{BN}}$ results is qualitatively similar to the pristine case, the three-fold configurations both show very non-ohmic behavior. In particular, the $\mathrm{Si}_{\mathrm{B}}$ configuration shows a diode-like behavior, where the current at $-0.5 \mathrm{~V}$ is six times larger than the current at $+0.5 \mathrm{~V}$, while the $\mathrm{Si}_{\mathrm{N}}$ configuration shows significant negative differential resistance (NDR) effect beyond $+0.2 \mathrm{~V}$ or $-0.3 \mathrm{~V}$.

As in the $(\mathrm{PbS})_{32}$ system above, the NDR effect depends strongly on the model chosen for the tip, and is not expected to be found if the DOS of the tip is roughly constant near the Fermi level. Therefore we do not expect the finding of NDR to be a robust result. However, the DOS for the different doping conditions is significantly different enough that the differential conductance curve should be a reliable differentiator. In figure 5.14 we have calculated the differential conductance for the three doped systems, using a model tip that has a constant density of states. Contrary to the above prediction, slight NDR is still found in the $\mathrm{Si}_{\mathrm{N}}$ system. This happens for two reasons. First, the DOS for $\mathrm{Si}_{\mathrm{N}}$ quickly goes to zero above the Fermi level, and so increasing bias does not increase the number of states that can contribute to conduction. Second, as the bias is increased, the states in $\mathrm{Si}_{\mathrm{N}}$ near the Fermi level are aligned with states in the tip deeper below the tip Fermi level, and these states see 


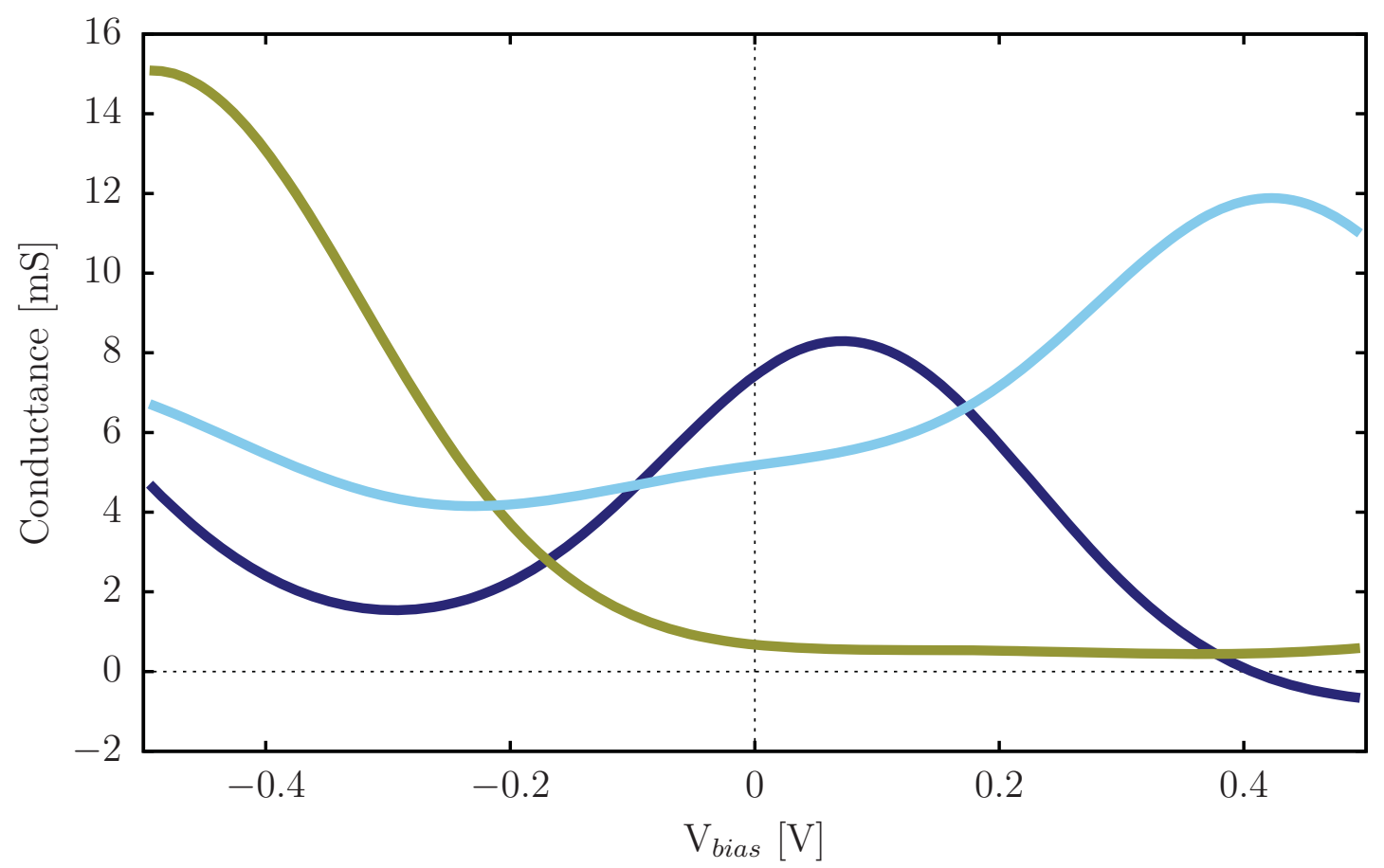

Figure 5.14: Differential conductance is calculated with a tip that has a constant density of states. $\mathrm{Si}_{\mathrm{N}}$ (solid purple), $\mathrm{Si}_{\mathrm{B}}$ (dashed green), and $\mathrm{Si}_{\mathrm{BN}}$ (dotted blue) each show unique conductance characteristics.

an increased tunnel barrier height. Still we find the characteristics for each system are quite distinctive. Therefore scanning tunneling spectroscopy (STS) should be a reliable method to differentiate the different doping sites, regardless of the details of the tip.

Finally we calculate constant current STM images at a bias of $100 \mathrm{mV}$ and set current of $1 \mathrm{nA}$ (figure 5.15). The signal for the $\mathrm{Si}_{\mathrm{B}}$ case is small, with the apparent height of the Si atom similar to the actual out-of-plane distance of the Si atom with respect to the surrounding $\mathrm{BN}$ monolayer. From the $I-V$ curves, we saw that the current above the $\mathrm{Si}$ atom in the $\mathrm{Si}_{\mathrm{B}}$ configuration was of a similar magnitude to the pristine $\mathrm{BN}$ monolayer. These pieces of information are in agreement, and we can conclude that the observed height of the $\mathrm{Si}$ atom in the $\mathrm{Si}_{\mathrm{B}}$ case is primarily attributed to the geometry of the $\mathrm{Si}_{\mathrm{B}}$ system. 


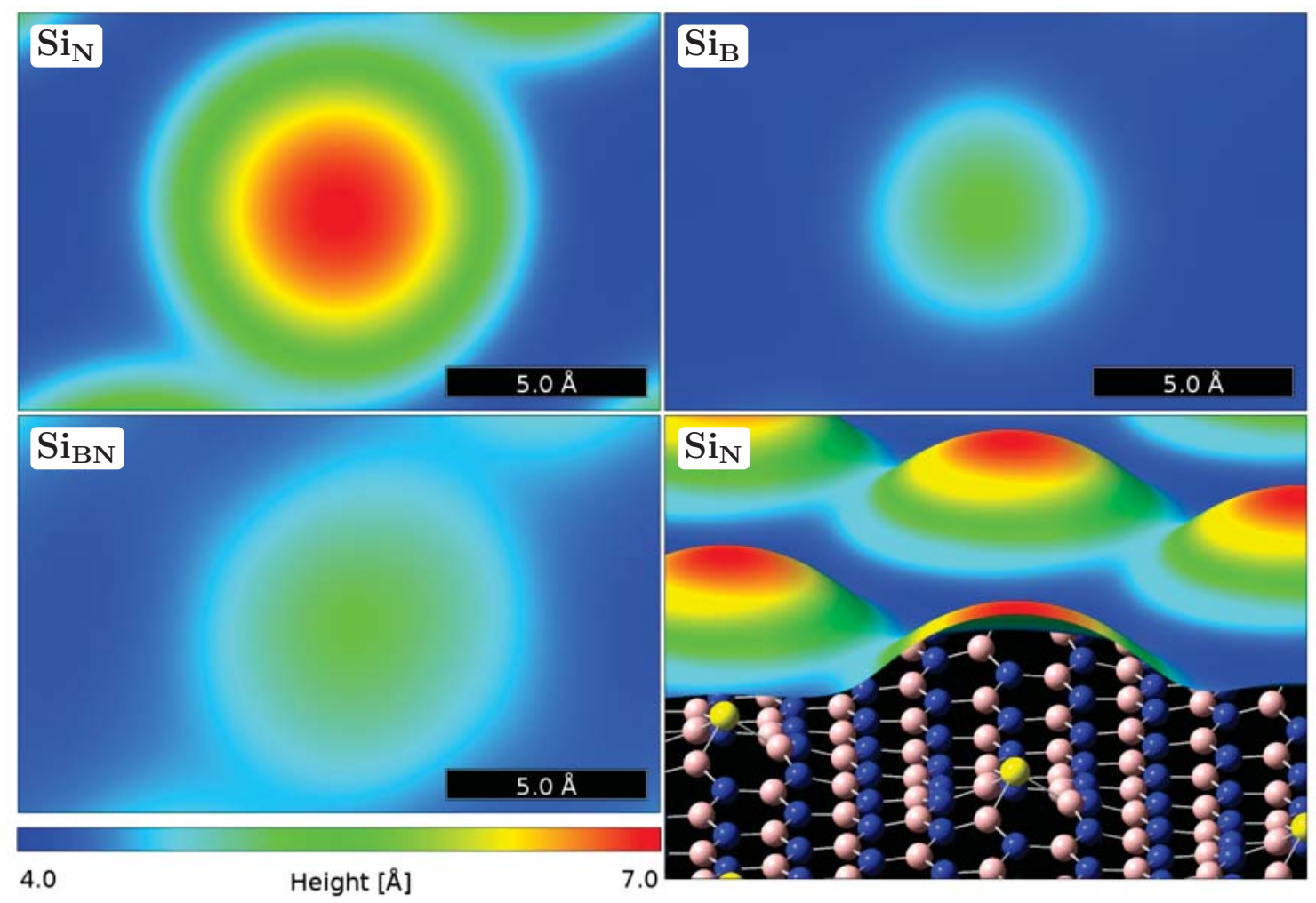

Figure 5.15: Constant current STM micrographs are presented for the $\mathrm{Si}_{\mathrm{N}}$, $\mathrm{Si}_{\mathrm{B}}$, and $\mathrm{Si}_{\mathrm{BN}}$ systems, calculated with a tip bias of $100 \mathrm{mV}$ and set current of $1 \mathrm{nA}$. Last panel is a cutaway view, showing the correspondence between the underlying geometry and the resulting constant-current surface.

For the $\mathrm{Si}_{\mathrm{BN}}$ system, the apparent height of the $\mathrm{Si}$ atom is similar to that of the $\mathrm{Si}_{\mathrm{B}}$ case, however the geometry cannot be responsible, because the $\mathrm{Si}$ atom is inplane with the surrounding $\mathrm{B}$ and $\mathrm{N}$ atoms. Therefore we can conclude here the image is entirely determined by the electronic structure of the sample. This is further supported by the observation that the calculated currents are more than an order of magnitude greater above the $\mathrm{Si}$ atom in the $\mathrm{Si}_{\mathrm{BN}}$ system than above the pristine sample.

Lastly, for the $\mathrm{Si}_{\mathrm{N}}$ system, the apparent height of the $\mathrm{Si}$ atom is greatest, at about $3 \AA$, which is roughly twice the out-of-plane distance of the Si atom with respect to the surrounding $\mathrm{BN}$ monolayer. The currents above the $\mathrm{Si}$ atom in the $\mathrm{Si}_{\mathrm{N}}$ system 
at low bias are more than an order of magnitude greater than the pristine case. This supports the conclusion that the apparent height of the $\mathrm{Si}$ atom in the $\mathrm{Si}_{\mathrm{N}}$ system must be attributed to both the geometry and the electronic structure of the sample.

\subsubsection{Conclusion}

Substitutional doping of $\mathrm{BN}$ monolayers with $\mathrm{Si}$ atoms is of interest for possible applications in future electronics. We investigated three dopant sites and demonstrate methods to distinguish them. We showed that the apparent height of the dopant is the same for the $\mathrm{Si}_{\mathrm{B}}$ and $\mathrm{Si}_{\mathrm{BN}}$ site, but that they are easily distinguished by scanning tunneling spectroscopy (STS) measurements. The $\mathrm{Si}_{\mathrm{N}}$ dopant site is most easily identified due to the very large apparent height and very unique STS spectra.

\subsection{Application to $\mathrm{MoS}_{2}$}

In recent work (Electronic stability and electron transport properties of atomic wires anchored on the $\mathrm{MoS}_{2}$ monolayer. Ashok Kumar, Douglas Banyai, P. K. Ahluwalia, Ravindra Pandey, and Shashi P. Karna. Phys.

Chem. Chem. Phys., 2014.) [6], we predicted the possibility of n-type and p-type doping of $2 \mathrm{D} \mathrm{MoS}_{2}$ through the addition of atomic wires of different composition. Specifically we looked at $\mathrm{Cu}, \mathrm{Ag}, \mathrm{Au}$, and Pt nanowires on one side of a $2 \mathrm{D} \mathrm{MoS}_{2}$ monolayer. In this section I will summarize some key results we obtained through the application of the SLSTM method. 


\subsubsection{Introduction}

Two-dimensional molybdenum disulfide $\left(\mathrm{MoS}_{2}\right)$ is a fascinating material. It has a hexagonal structure and, like graphene, can be exfoliated from bulk with the so-called "Scotch-tape method." Unlike graphene, $2 \mathrm{D} \mathrm{MoS}_{2}$ is a direct-gap semiconductor with a $\sim 1.5 \mathrm{eV}$ band gap, making it a material of much interest for possible usage in digital logic devices.

\subsubsection{Model}

The $\mathrm{MoS}_{2}$ monolayer was simulated using periodic boundary conditions and a $(4 \times 4)$ supercell in-plane and with $15 \AA$ separation between layers. Energy calculations were performed using the SIESTA code within density functional theory (DFT) under the generalized gradient approximation (GGA) with the Perdew-Burke-Ernzerhof (PBE) exchange-correlation functional. The pristine monolayer was fully relaxed until residual forces were less than $0.01 \mathrm{eV} \AA^{-1}$. The lattice constant was found to be $3.23 \AA$. The lattice constants for the atomic wires were found to be $2.42,2.65,2.60$, and 2.50 $\AA$ for atomic wires of $\mathrm{Cu}, \mathrm{Ag}, \mathrm{Au}$, and $\mathrm{Pt}$, respectively. This allows for the use of a $(5 \times 1)$ supercell for the atomic wires, resulting in less than $6 \%$ mismatch in the co-periodic lattices. The $\mathrm{MoS}_{2}$ monolayer with attached $\mathrm{Au}$ atomic wire is shown in figure 5.16, and is representative of the geometry for each of the atomic wire systems.

Electronic transport properties were calculated with the STM probe (tip) $5 \AA$ above the atomic wire, centered on one of the wire atoms. The tip is represented by a $\mathrm{Au}_{43}$ cluster. 

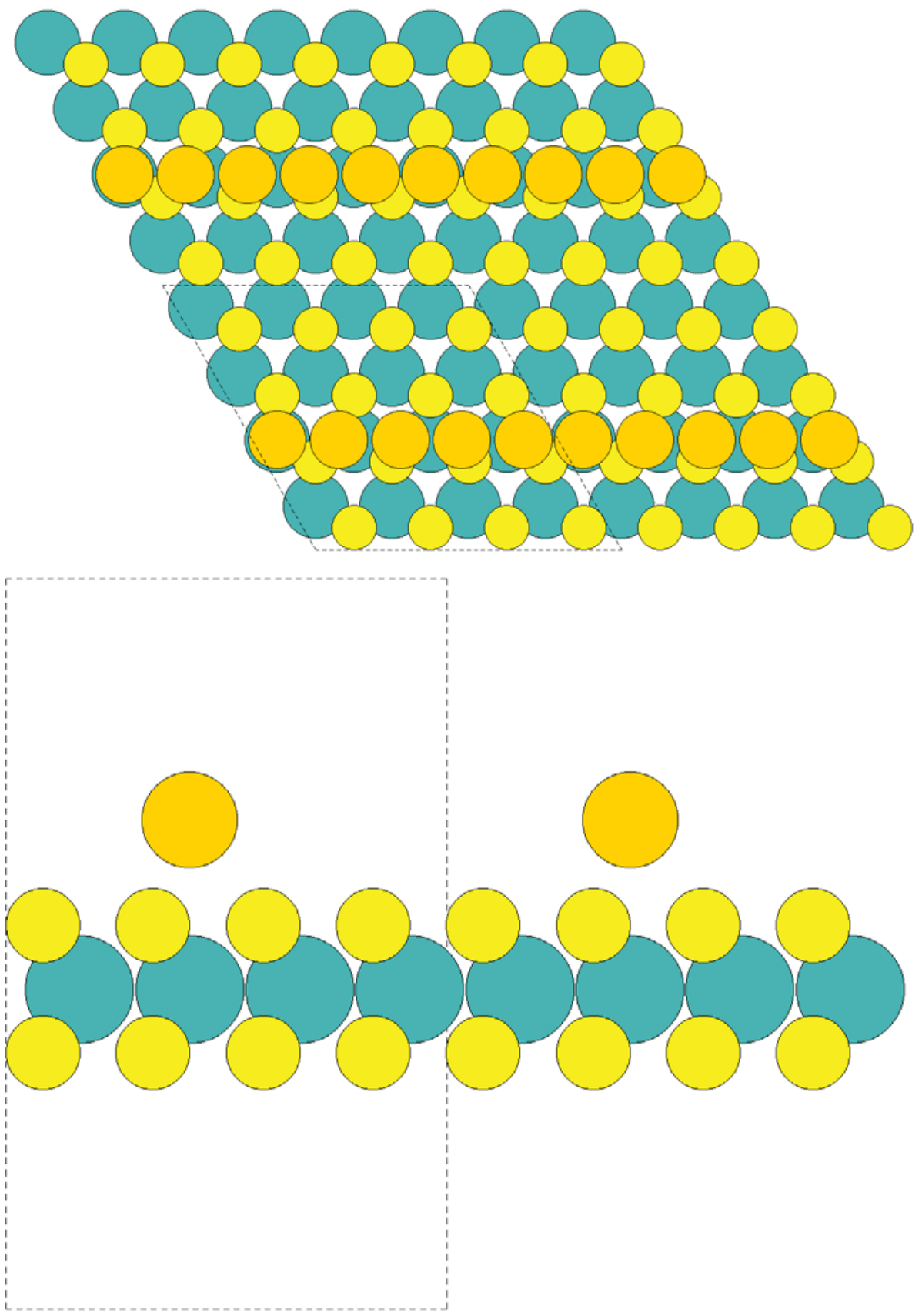

Figure 5.16: Top- and side-views of the $\mathrm{MoS}_{2}$ monolayer with an attached $\mathrm{Au}$ atomic wire. The extent of the supercell is indicated with dashed lines. Two repetitions of the supercell in the $\mathrm{a}$ and $\mathrm{b}$ directions are shown for clarity. Mo atoms are blue, S atoms are yellow, and Au atoms are orange. 


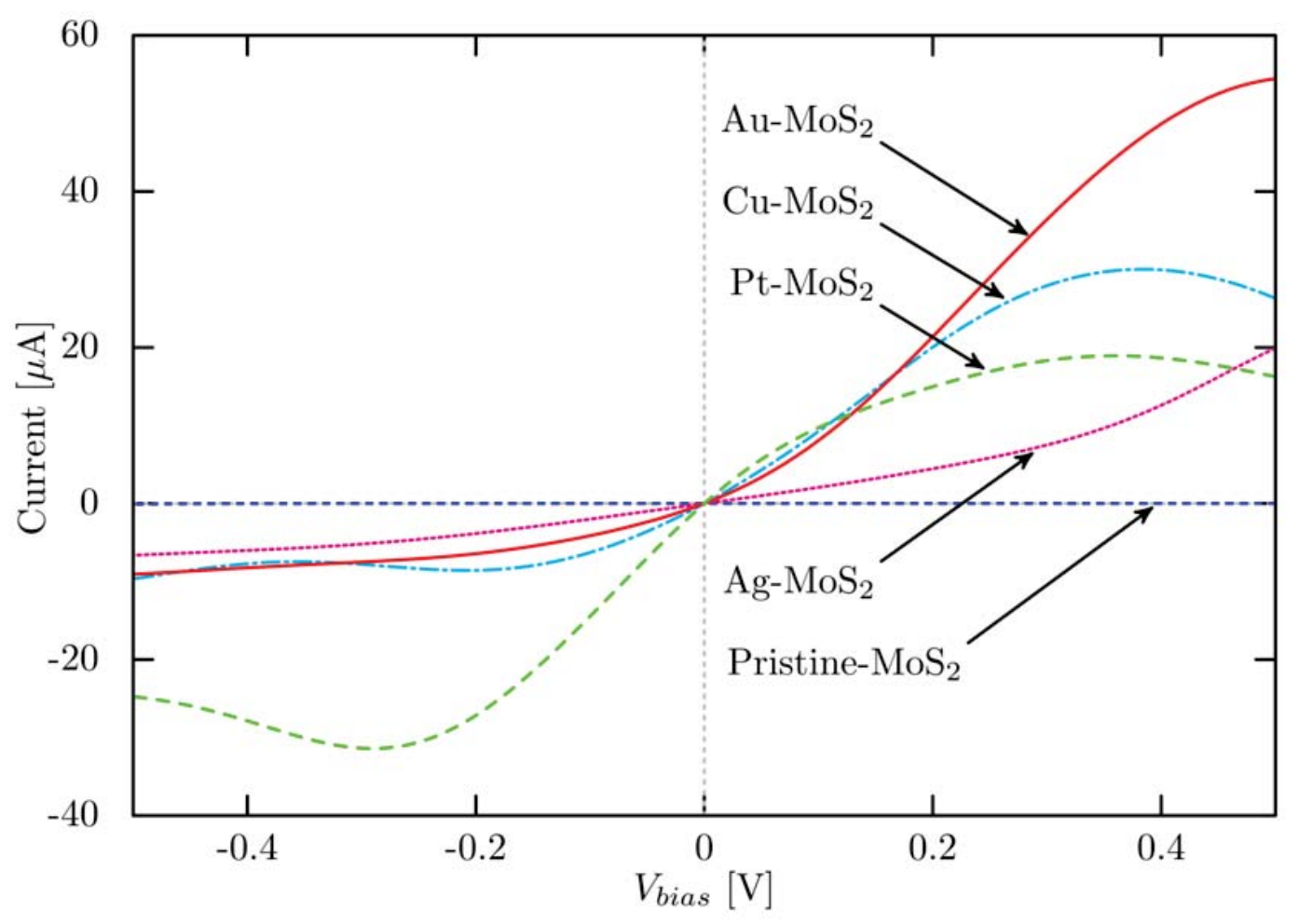

Figure 5.17: Current is calculated as a function of bias voltage with a tip $5 \AA$ above the pristine $\mathrm{MoS}_{2}$ monolayer or $5 \AA$ above the metal atoms of the atomic-wire on $\mathrm{MoS}_{2}$ systems. The $\mathrm{Au}, \mathrm{Cu}$, and $\mathrm{Ag}$ systems show n-type behavior, with enhanced current for a positively biased sample (negatively biased tip), while the Pt system shows p-type behavior.

\subsubsection{Results and Discussion}

Figure 5.17 shows calculated current-voltage measurements in the STM-like configuration for the pristine $\mathrm{MoS}_{2}$ monolayer, and for each monolayer+atomic-wire system. As expected, the pristine monolayer, with its sizeable band gap, admits a negligible amount of current relative to the samples with atomic wires attached. Interestingly, there is a qualitative difference between the results for the Pt system and each of the other systems. The non-Pt systems, especially the Au system, show enhanced 
current in the positive-bias direction relative to the negative-bias results, similar to n-type semiconductors. Conversely, the Pt system shows decreased current in the positive-bias direction relative to the negative-bias results, indicating p-type behavior. Mulliken charge analysis provides some insight into the origin of this results, as the $\mathrm{Pt}$ wire gains roughly $0.25 \mathrm{e}$ /atom from the substrate, while the other atomic wires give roughly $0.05 \mathrm{e} /$ atom to the substrate.

\subsubsection{Conclusion}

Two-dimensional $\mathrm{MoS}_{2}$ is a promising semiconducting material with a direct band gap. We found the $\mathrm{MoS}_{2}$ monolayer can be effectively doped by attaching atomic wires to the surface of the monolayer. p-type doping was observed with the addition

of a $\mathrm{Pt}$ monowire, while $\mathrm{Cu}, \mathrm{Ag}$, and $\mathrm{Au}$ monowires resulted in n-type doping. This result, along with the observed excess charges on the surface of the monolayer, lead us to propose these materials for use in catalysis. 



\section{Chapter 6}

\section{Future Work and Open Questions}

\subsection{Overview}

In this thesis we have investigated - and developed new methods for investigatingcertain classes of single electron devices (SEDs). Such devices have the potential for being the basis of the next generation of digital circuits. In addition to any possible commercializability of these designs, these devices are useful testbeds for exploring single-electronics. Fundamental questions remain regarding such devices, and many avenues remain for furthering this research into the behavior of such devices. They can be categorized into three categories: investigating and improving the characterization of the detailed behavior of individual junctions, improving the algorithms and tools for exploring the collective behavior of many-junction devices, and exploring and characterizing the behavior of ensembles of devices. The next few sections we will describe some of these questions and proposed methods to investigate them.

We have also described a new method for investigating scanning tunneling microscopy 
and scanning tunneling spectroscopy (STM/STS), which we call SLSTM. We have shown this method reproduces some experimental results quite well, including some that are not well described by similar methods. However there is still further work to be done to assess the accuracy of the method relative to other state of the art methods, and to search for systems that demonstrate any limitations of the method. These ideas will be expanded upon in the last section of this chapter.

\subsection{Detailed Behavior of Individual Junctions}

Characterizing devices requires understanding the working of the details, which primarily means the changes in energy as described by the capacitances, and the tunneling currents as described by the tunnelling resistances.

In this work we improved upon previous modeling efforts by employing state of the art methods to calculate capacitances in the model systems. These calculations can be improved in a number of ways. The fidelity of the geometrical description of the devices can be improved. For example, we modeled all the island electrodes as perfect spheres embedded in a uniform dielectric, and these geometric models could be made more detailed. The existence of sharp protuberances or edges would affect capacitances, and the associated increased electric fields could affect tunneling probabilities.

More interestingly, we would like to extend these methods for use with systems containing semiconductor islands and electrodes. This raises many questions, including whether capacitance matrices can fully determine the change in energy when an electron tunnels between semiconductors, and whether these methods designed for calculating capacitances of perfect conductors can be used for calculating capacitances 
of semiconductors. Additionally, the so-called Orthodox Theory [20] underpinning our kinetic Monte Carlo solver [3] makes many approximations in the derivation of the resulting rate equations. The most obviously troublesome is the assumption of a constant density of states, which has been investigated previously in a limited fashion $[33,97]$. Orthodox theory also assumes the transition matrix can be approximated by a constant, which is independent of the initial or final states. This may be a reasonable approximation for the states near the Fermi level in a conductor, but should be revisited for the case of semiconductor quantum dots.

We also investigated the tunneling currents between the islands using density functional theory (DFT) by calculating conductance between nanoscale gold clusters. In this work we focused on the low-bias conductance, and it would be valuable to investigate whether the moderate- and high-bias conductance follows the same trends we found for low-bias. The methods we used are not suitable for high-bias calculations, and it would be valuable to explore these questions with a more robust tool. Additionally, these calculations focused on pairs of isolated clusters, which leaves two prominent lines of investigations. These devices are dense with islands, and so it would be valuable to perform calculations to investigate the role of neighbor islands. Additionally the islands are attached to or even embedded in a substrate. This raises a question of whether a nearby insulating substrate can affect the rate at which electrons tunnel from one island directly to another. Even more interesting, is the question of whether substrate assisted tunneling occurs, where states associated with the substrate play an essential role in the tunneling process. An excellent starting point would be to study two gold nanoclusters adsorbed on a boron nitride nanotube surface, investigating whether the clusters create states in the band gap of the nanotube, and if the nanotube modifies the conductance of the junction between the clusters. 


\subsection{Algorithms and Tools for Investigating Device- level Properties}

The most important properties of a SED device are the zero-temperature threshold voltage $V_{t h}$, the gate periodicity $\Delta V_{g}$, and the resulting Coulomb diamond (CD) structure. We currently use the kinetic Monte Carlo (KMC) algorithm to investigate these properties, which is a powerful technique that allows for simulating the dynamic evolution of a system. However, the above mentioned properties are not dynamic, and computational time could be saved by developing algorithms which calculate these properties without simulating the dynamics of the system. Such a tool would enable calculating the properties of ensembles of devices.

There are two approaches that appear particularly promising. One would be to use our existing $\mathrm{KMC}$ framework to determine zero-temperature Coulomb diamond by mapping out the regions in $V_{s d^{-}} V_{g}$ space where no steady state current is possible. This would require developing a method for differentiating a steady-state current from a transient avalanche of electrons flowing through the system due to a change in applied bias. Another approach would be to extend the master equation (ME) approach to systems of more than one island. From preliminary investigations, it appears the ME approach for a chain of multiple islands would yield a sparse matrix equation that could be solved numerically. The mathematics would be more complicated than the single-island ME, but the solution speed would be much faster than KMC. 


\subsection{Exploring Ensembles of Devices}

Before SEDs are mass produced, we would like to know not just how one device might behave, but rather we would like to know the distributions of the resulting device properties. In integrated circuit devices such as MOSFET transistors, small differences in otherwise identical devices create variations in the behavior of individual devices. These variations limit the performance and efficiency of the integrated circuit as a whole by requiring operating the device within the limits of the weakest link. This problem is expected to be exacerbated with circuits made from SEDs as they are exquisitely sensitive to the capacitances of the islands, which is affected not only by the device itself but also by all the other devices and wires nearby. When we consider devices such as those described in this thesis, created with randomly placed islands, we can expect much greater variation between individual devices. This raises the question of whether existing models for creating very large scale integrated circuits (VLSI) will be able to accommodate such devices, or whether new fault-tolerant-type models will have to be developed.

We can investigate this by creating ensembles of devices and measuring their properties. In creating model SEDs, we select some number of islands with radii and junction widths picked from distributions with a given mean and variance. By cal-

culating the dependence of system properties on the input distributions, we may be able gain insight into ways to engineer devices to optimize various system properties. 


\subsection{Characterizing the Accuracy and Limitations of SLSTM}

In this work we developed a novel algorithm for calculating scanning tunneling microscopy/spectroscopy (STM/STS) results from projected electronic density of states (PDOS) data, and implemented the algorithm in a new STM calculation package, SLSTM. Further work should be done to characterize the performance and limitations of this new algorithm, specifically in comparison to existing methods.

Existing state of the art methods for calculating STM/STS properties include the Green's function-based method of Paz et al. [72] and the elastic scattering quantum chemistry method [73]. So far we have only been able to compare SLSTM to results from these methods as published in the literature. However, these methods are implemented in quantum chemistry codes such as SIESTA [98, 99] which are able to export the PDOS data required as input to SLSTM. Calculations of STM/STS properties should be performed using the method of Paz et al., with the resulting PDOS saved. Comparisons can then be made between these results and calculations performed with SLSTM which use the same PDOS as input. This would allow direct comparisons between the PAZ STM algorithm and the SLSTM algorithm, without the current confounding factors of not having an identical foundation for the comparisons. Additionally, while the $\operatorname{Si}(111)-(7 \times 7)$ reconstructed surface is a great test case with lots of published results to compare to, having the ability to make these direct comparisons would enable testing a wider variety of systems. 


\section{References}

[1] J. A. Jaszczak, M. A. Savaikar, D. R. Banyai, B. Hao, D. Zhang, P. L. Bergstrom, A.-P. Li, J. C. Idrobo, and Y. K. Yap. Simulation of Charge Transport in Disordered Assemblies of Metallic Nano-Islands: Application to Boron-Nitride Nanotubes Functionalized with Gold Quantum Dots. MRS Proceedings, 1700:1728, July 2014.

[2] C. H. Lee, S. Qin, M. A. Savaikar, J. Wang, B. Hao, D. Zhang, D. Banyai, J. A. Jaszczak, K. W. Clark, and J. C. Idrobo. Room-Temperature Tunneling Behavior of Boron Nitride Nanotubes Functionalized with Gold Quantum Dots. Advanced Materials, 25(33):4544-4548, 2013.

[3] M. A. Savaikar, D. Banyai, P. L. Bergstrom, and J. A. Jaszczak. Simulation of charge transport in multi-island tunneling devices: Application to disordered one-dimensional systems at low and high biases. Journal of Applied Physics, 114(11):114504, 2013.

[4] S. K. Gupta, H. He, D. Banyai, A. K. Kandalam, and R. Pandey. Electron tunneling characteristics of a cubic quantum dot, $(\mathrm{PbS})_{32}$. The Journal of Chemical Physics, 139(24):244307, December 2013.

[5] S. K. Gupta, H. He, D. Banyai, M. Si, R. Pandey, and S. P. Karna. Effect of Si 
doping on the electronic properties of BN monolayer. Nanoscale, 6:5526-5531, 2014.

[6] A. Kumar, D. Banyai, P. K. Ahluwalia, R. Pandey, and S. P. Karna. Electronic stability and electron transport properties of atomic wires anchored on the $\mathrm{MoS}_{2}$ monolayer. Phys. Chem. Chem. Phys., 16:20157-20163, 2014.

[7] G. E. Moore. Cramming more components on electronic circuits. Electronics, 38, April 1965.

[8] E. A. Johannessen, T. B. Tang, L. Wang, L. Cui, M. Ahmadian, N. Aydin, A. Astaras, A. F. Murray, B. W. Flynn, T. Aslan, S. P. Beaumont, D. R. S. Cumming, and J. M. Cooper. An Ingestible Electronic Pill for Real Time Analytical Measurements of the Gastro-Intestinal Tract. In Micro Total Analysis Systems 2001: Proceedings of the $\mu T A S$ 2001, pages 181-183. Micro Total Analysis Systems 2002, 2002.

[9] B. Tebrean, S. Crisan, I. G. Tarnovan, and T. E. Crisan. Novel Drug Delivery Systems-Method Review. Acta Electrotechnica, 52:49-54, 2011.

[10] C. C. Mann. The End of Moore's Law? MIT Technology Review, 2000.

[11] C. J. Gorter. A possible explanation of the increase of the electrical resistance of thin metal films at low temperatures and small field strengths. Physica, 17(8):777-780, August 1951.

[12] D. V. Averin and K. K. Likharev. Coulomb blockade of single-electron tunneling, and coherent oscillations in small tunnel junctions. Journal of Low Temperature Physics, 62(3-4):345-373, February 1986.

[13] T. A. Fulton and G. J. Dolan. Observation of single-electron charging effects in small tunnel junctions. Physical Review Letters, 59:109-112, 1987. 
[14] J. Shirakashi, K. Matsumoto, N. Miura, and M. Konagai. Single-electron charging effects in $\mathrm{Nb} / \mathrm{Nb}$ oxide-based single-electron transistors at room temperature. Applied Physics Letters, 72(15):1893-1895, 1998.

[15] Y. A. Pashkin, Y. Nakamura, and J. S. Tsai. Room-temperature Al singleelectron transistor made by electron-beam lithography. Applied Physics Letters, 76(16):2256-2258, 2000.

[16] V. Ray, R. Subramanian, P. Bhadrachalam, L.-C. Ma, C.-U. Kim, and S. J. Koh. CMOS-compatible fabrication of room-temperature single-electron devices. Nature Nanotechnology, 3(10):603-608, September 2008.

[17] D. D. Cheam, P. S. K. Karre, M. Palard, and P. L. Bergstrom. Mass Production of Room Temperature Single Electron Transistors using Step \& Flash Imprint Lithography and Lift-Off Technique. In Nanotechnology, 2008. NANO '08. 8th IEEE Conference on, pages 175-178, 2008.

[18] R. Parthasarathy, X.-M. Lin, and H. Jaeger. Electronic Transport in Metal Nanocrystal Arrays: The Effect of Structural Disorder on Scaling Behavior. Physical Review Letters, 87(18):186807, October 2001.

[19] D. V. Averin and K. K. Likharev. Single-electronics: A correlated transfer of single electrons and cooper pairs in systems of small tunnel junctions,". Mesoscopic phenomena in solids, 30:173-271, 1991.

[20] K. K. Likharev. Single-electron devices and their applications. Proceedings of the IEEE, 87(4):606-632, 1999.

[21] G. Binnig, H. Rohrer, C. Gerber, and E. Weibel. Surface studies by scanning tunneling microscopy. Physical Review Letters, 49(1):57, 1982. 
[22] A. Ribes and C. Caremoli. Salomé platform component model for numerical simulation. In COMPSAC 0\%: Proceeding of the 31st Annual International Computer Software and Applications Conference, pages 553-564, Washington, DC, 2007. IEEE Computer Society.

[23] CSC - IT Center for Science. Elmer Manuals. http://www.csc.fi/elmer/.

[24] A. M. Ionescu and H. Riel. Tunnel field-effect transistors as energy-efficient electronic switches. Nature, 479(7373):329-337, November 2011.

[25] I. Ferain, C. A. Colinge, and J.-P. Colinge. Multigate transistors as the future of classical metal-oxide-semiconductor field-effect transistors. Nature, 479(7373):310-316, November 2011.

[26] P. Sheng and B. Abeles. Voltage-induced tunneling conduction in granular metals at low temperatures. Physical Review Letters, 28(1):34, 1972.

[27] A. S. Cordan, A. Goltzene, Y. Herve, M. Mejias, C. Vieu, and H. Launois. Electron transport in metallic dot arrays: Effect of a broad dispersion in the tunnel junction dimensions. Journal of Applied Physics, 84(7):3756-3763, October 1998.

[28] P. S. K. Karre, P. L. Bergstrom, G. Mallick, and S. P. Karna. Room temperature operational single electron transistor fabricated by focused ion beam deposition. Journal of Applied Physics, 102(2):024316-024316-4, 2007.

[29] K. Yano, T. Ishii, T. Hashimoto, T. Kobayashi, F. Murai, and K. Seki. Roomtemperature single-electron memory. IEEE Transactions on Electron Devices, 41(9):1628-1638, 1994.

[30] R. G. Knobel and A. N. Cleland. Nanometre-scale displacement sensing using a single electron transistor. Nature, 424(6946):291-293, July 2003. 
[31] A. B. Bortz, M. H. Kalos, and J. L. Lebowitz. A new algorithm for Monte Carlo simulation of Ising spin systems. Journal of Computational Physics, 17(1):10-18, January 1975.

[32] M. Kotrla. Numerical simulations in the theory of crystal growth. Computer Physics Communications, 1996.

[33] C. Wasshuber. Computational single-electronics. Springer, Wien New York, 2001.

[34] E. Pisler and T. Adhikari. Numerical Calculation of Mutual Capacitance between Two Equal Metal Spheres - Abstract - Physica Scripta - IOPscience. Physica Scripta, 2(3):81-83, February 2007.

[35] J. Lekner. Capacitance coefficients of two spheres. Journal of Electrostatics, 2011.

[36] J. G. Simmons. Generalized Formula for the Electric Tunnel Effect between Similar Electrodes Separated by a Thin Insulating Film. Journal of Applied Physics, 34(6):1793, 1963.

[37] D. D. Cheam, P. Karre, M. Palard, and P. L. Bergstrom. Step and flash imprint lithography for quantum dots based room temperature single electron transistor fabrication. Microelectronic Engineering, 2009.

[38] A. J. Quinn, M. Biancardo, L. Floyd, M. Belloni, P. R. Ashton, J. A. Preece, C. A. Bignozzi, and G. Redmond. Analysis of charge transport in arrays of 28 kDa nanocrystal gold molecules. Journal of Materials Chemistry, 15(41):44034407, 2005.

[39] C. A. Neugebauer and M. B. Webb. Electrical Conduction Mechanism in Ultrathin, Evaporated Metal Films. Journal of Applied Physics, 1962. 
[40] R. Parthasarathy, X.-M. Lin, K. Elteto, T. Rosenbaum, and H. Jaeger. Percolating through Networks of Random Thresholds: Finite Temperature Electron Tunneling in Metal Nanocrystal Arrays. Physical Review Letters, 92(7):076801, February 2003.

[41] A. Rimberg, T. Ho, and J. Clarke. Scaling Behavior in the Current-Voltage Characteristic of One- and Two-Dimensional Arrays of Small Metallic Islands. Physical Review Letters, 74(23):4714-4717, June 1995.

[42] A. Bezryadin, R. M. Westervelt, and M. Tinkham. Threshold transport properties of self-assembled 1D chains of conducting nanoparticles. Appl. Phys. Lett., 74(cond-mat/9810235):2699, 1999.

[43] A. Middleton and N. Wingreen. Collective transport in arrays of small metallic dots. Physical Review Letters, 71(19):3198-3201, November 1993.

[44] A. N. Aleshin, H. J. Lee, S. H. Jhang, H. S. Kim, K. Akagi, and Y. W. Park. Coulomb-blockade transport at low temperature in quasi-one dimensional polymer nanofibers. Physical Review B, 72(cond-mat/0506069):153202. 4 p, June 2005.

[45] V. V. Deshpande, M. Bockrath, L. I. Glazman, and A. Yacoby. Electron liquids and solids in one dimension. Nature, 464(7286):209-216, March 2010.

[46] K. Nabors and J. White. Fastcap: A multipole accelerated 3-d capacitance extraction program. Computer-Aided Design of Integrated Circuits and Systems, IEEE Transactions on, 10(11):1447-1459, 1991.

[47] S. Kapur and D. E. Long. Ies 3: A fast integral equation solver for efficient 3-dimensional extraction. In Proceedings of the $199^{\prime 7}$ IEEE/ACM international conference on Computer-aided design, pages 448-455. IEEE Computer Society, 1997. 
[48] W. Shi, J. Liu, N. Kakani, and T. Yu. A fast hierarchical algorithm for threedimensional capacitance extraction. Computer-Aided Design of Integrated Circuits and Systems, IEEE Transactions on, 21(3):330-336, 2002.

[49] W. Yu and Z. Wang. Enhanced qmm-bem solver for three-dimensional multipledielectric capacitance extraction within the finite domain. Microwave Theory and Techniques, IEEE Transactions on, 52(2):560-566, 2004.

[50] W. Chai, D. Jiao, and C.-K. Koh. A direct integral-equation solver of linear complexity for large-scale 3d capacitance and impedance extraction. In Design Automation Conference, 2009. DAC'09. 46th ACM/IEEE, pages 752-757. IEEE, 2009.

[51] M. Naghed and I. Wolff. Equivalent capacitances of coplanar waveguide discontinuities and interdigitated capacitors using a three-dimensional finite difference method. Microwave Theory and Techniques, IEEE Transactions on, 38(12):1808-1815, 1990.

[52] Y. L. Le Coz and R. B. Iverson. A stochastic algorithm for high speed capacitance extraction in integrated circuits. Solid-State Electronics, 35(7):1005-1012, 1992.

[53] T. A. El-Moselhy, I. M. Elfadel, and L. Daniel. A hierarchical floating random walk algorithm for fabric-aware 3d capacitance extraction. In Proceedings of the 2009 International Conference on Computer-Aided Design, pages 752-758. ACM, 2009.

[54] W. Yu, H. Zhuang, C. Zhang, G. Hu, and Z. Liu. Rwcap: A floating random walk solver for 3-d capacitance extraction of very-large-scale integration interconnects. Computer-Aided Design of Integrated Circuits and Systems, IEEE Transactions on, 32(3):353-366, 2013. 
[55] K. Zhai, W. Yu, and H. Zhuang. Gpu-friendly floating random walk algorithm for capacitance extraction of vlsi interconnects. In Proceedings of the Conference on Design, Automation and Test in Europe, pages 1661-1666. EDA Consortium, 2013.

[56] T. Heinzel. Capacitance Matrix and Electrostatic Energy, pages 349-352. WileyVCH Verlag GmbH, 2008.

[57] C. B. Duke. Theory of Metal-Barrier-Metal Tunneling. In Tunneling phenomena in solids, pages 31-46. Springer US, Boston, MA, 1969.

[58] B. Hao and A. Asthana and P. K. Hazaveh and P. L. Bergstrom and D. Banyai and M. A. Savaikar and J. A. Jaszczak and Y. K. Yap. Flexible channels for tunneling field effect transistors by quantum dots functionalized boron nitride nanotubes. in manuscript, 2014.

[59] M. Born and R. Oppenheimer. On the Quantum Theory of Molecules (translation by S. M. Blinder). Annalen der Physik, 84:457-484, 1927.

[60] P. Hohenberg and W. Kohn. Inhomogeneous electron gas. Phys. Rev. B, 136(3B):B864-B871, 1964.

[61] M. J. Frisch, G. W. Trucks, H. B. Schlegel, G. E. Scuseria, M. A. Robb, J. R. Cheeseman, G. Scalmani, V. Barone, B. Mennucci, G. A. Petersson, H. Nakatsuji, M. Caricato, X. Li, H. P. Hratchian, A. F. Izmaylov, J. Bloino, G. Zheng, J. L. Sonnenberg, M. Hada, M. Ehara, K. Toyota, R. Fukuda, J. Hasegawa, M. Ishida, T. Nakajima, Y. Honda, O. Kitao, H. Nakai, T. Vreven, J. A. Montgomery, Jr., J. E. Peralta, F. Ogliaro, M. Bearpark, J. J. Heyd, E. Brothers, K. N. Kudin, V. N. Staroverov, R. Kobayashi, J. Normand, K. Raghavachari, A. Rendell, J. C. Burant, S. S. Iyengar, J. Tomasi, M. Cossi, N. Rega, J. M. Millam, M. Klene, J. E. Knox, J. B. Cross, V. Bakken, C. Adamo, J. Jaramillo, R. Gomperts, R. E. Stratmann, O. Yazyev, A. J. Austin, R. Cammi, C. Pomelli, 
J. W. Ochterski, R. L. Martin, K. Morokuma, V. G. Zakrzewski, G. A. Voth, P. Salvador, J. J. Dannenberg, S. Dapprich, A. D. Daniels, Ö. Farkas, J. B. Foresman, J. V. Ortiz, J. Cioslowski, and D. J. Fox. Gaussian 09 Revision D.01. Gaussian Inc. Wallingford CT 2009.

[62] A. D. Becke. Density-functional thermochemistry. iii. the role of exact exchange. The Journal of Chemical Physics, 98(7):5648-5652, 1993.

[63] P. J. Hay and W. R. Wadt. Ab initio effective core potentials for molecular calculations. potentials for the transition metal atoms sc to hg. The Journal of Chemical Physics, 82(1):270-283, 1985.

[64] W. R. Wadt and P. J. Hay. Ab initio effective core potentials for molecular calculations. potentials for main group elements na to bi. The Journal of Chemical Physics, 82(1):284-298, 1985.

[65] P. J. Hay and W. R. Wadt. Ab initio effective core potentials for molecular calculations. potentials for $\mathrm{k}$ to au including the outermost core orbitals. The Journal of Chemical Physics, 82(1):299-310, 1985.

[66] T. H. Dunning, P. J. Hay, and H. F. Schaefer. Gaussian basis sets for molecular calculations. In H. F. Schaefer, editor, Methods of Electronic Structure Theory, volume 3 of Modern Theoretical Chemistry, pages 1-27. Springer US, 1977.

[67] K. Burke. The abc of dft. Department of Chemistry, University of California, 2007.

[68] J. Tersoff and D. R. Hamann. Theory of the scanning tunneling microscope. Physical Review B, 31(2):805-813, January 1985.

[69] R. M. Tromp, R. J. Hamers, and J. E. Demuth. Atomic and electronic contributions to $\mathrm{Si}(111)-(7 \times 7)$ scanning-tunneling-microscopy images. Physical Review B, 34(2):1388-1391, July 1986. 
[70] J. Tersoff and D. R. Hamann. Theory and Application of the Scanning Tunneling Microscope. Physical Review Letters, 50(25):1998-2001, 1983.

[71] N. D. Lang. Spectroscopy of single atoms in the scanning tunneling microscope. Physical Review B, 34(8):5947, 1986.

[72] O. Paz, I. Brihuega, J. Gómez-Rodríguez, and J. Soler. Tip and Surface Determination from Experiments and Simulations of Scanning Tunneling Microscopy and Spectroscopy. Physical Review Letters, 94(5):056103, February 2005.

[73] C. Chavy, C. Joachim, and A. Altibelli. Interpretation of STM images: $\mathrm{C}_{60}$ on the gold (110) surface. Chemical Physics Letters, 214(6):569-575, November 1993.

[74] J. Bardeen. Tunnelling from a Many-Particle Point of View. Physical Review Letters, pages 1-3, December 1960.

[75] J. Dunham. The Wentzel-Brillouin-Kramers Method of Solving the Wave Equation. Physical Review, 41(6):713-720, September 1932.

[76] H. He, R. Pati, R. Pandey, and S. P. Karna. Spin-dependent electron transport along a molecular wire in a metal (probe)-vacuum-molecule-metal system: the effect of the size and the shape of the probe tip. In 5th IEEE Conference on Nanotechnology, 2005., pages 80-83. IEEE, 2005.

[77] J. Tersoff, M. Cardillo, and D. Hamann. Sensitivity of helium diffraction to surface geometry. Physical Review B, 32(8):5044-5050, October 1985.

[78] G. Binnig, H. Rohrer, C. Gerber, and E. Weibel. $7 \times 7$ Reconstruction on Si(111) Resolved in Real Space. Physical Review Letters, 50(2):120-123, January 1983.

[79] K. Takayanagi. Structural analysis of $\mathrm{Si}(111)-(7 \times 7)$ by UHV-transmission electron diffraction and microscopy. Journal of Vacuum Science 85 Technology A: Vacuum, Surfaces, and Films, 3(3):1502, May 1985. 
[80] P. Sutter, P. Zahl, E. Sutter, and J. Bernard. Energy-Filtered Scanning Tunneling Microscopy using a Semiconductor Tip. Physical Review Letters, 90(16):166101, April 2003.

[81] G. Kresse and J. Hafner. Ab Initio Molecular Dynamics for Liquid Metals. Physical Review B, 47:558, 1993.

[82] G. Kresse and J. Hafner. Ab Initio Molecular-Dynamics Simulation of the LiquidMetal-Amorphous-Semiconductor Transition in Germanium. Physical Review B, 49:14251, 1994.

[83] G. Kresse. Efficient iterative schemes for ab initio total-energy calculations using a plane-wave basis set. Physical Review B, 54(16):11169-11186, October 1996.

[84] P. E. Blöchl. Projector augmented-wave method. Physical Review B, 50(24):17953-17979, December 1994.

[85] J. P. Perdew, K. Burke, and M. Ernzerhof. Generalized Gradient Approximation Made Simple. Physical Review Letters, 77(18):3865-3868, October 1996.

[86] O. Custance, S. Brochard, I. Brihuega, E. Artacho, J. Soler, A. M. Baró, and J. Gómez-Rodríguez. Single adatom adsorption and diffusion on $\operatorname{Si}(111)-(7 \times 7)$ surfaces: Scanning tunneling microscopy and first-principles calculations. Physical Review B, 67(23):235410, June 2003.

[87] M. Lantz, H. Hug, P. van Schendel, R. Hoffmann, S. Martin, A. Baratoff, A. Abdurixit, H. J. Güntherodt, and C. Gerber. Low Temperature Scanning Force Microscopy of the Si(111)-(7×7) Surface. Physical Review Letters, 84(12):26422645, March 2000.

[88] S. Y. Jang, Y. M. Song, H. S. Kim, Y. J. Cho, Y. S. Seo, G. B. Jung, C.-W. Lee, J. Park, M. Jung, J. Kim, B. Kim, J.-G. Kim, and Y.-J. Kim. Three Synthetic Routes to Single-Crystalline PbS Nanowires with Controlled Growth Direction 
and Their Electrical Transport Properties. ACS Nano, 4(4):2391-2401, April 2010.

[89] L. Bakueva, S. Musikhin, M. A. Hines, T.-W.F. Chang, M. Tzolov, G. D. Scholes, and E. H. Sargent. Size-tunable infrared (1000-1600 nm) electroluminescence from $\mathrm{PbS}$ quantum-dot nanocrystals in a semiconducting polymer. Applied Physics Letters, 82(17):2895-2897, 2003.

[90] A. H. Khan, U. Thupakula, A. Dalui, S. Maji, A. Debangshi, and S. Acharya. Evolution of Long Range Bandgap Tunable Lead Sulfide Nanocrystals with Photovoltaic Properties. The Journal of Physical Chemistry C, 117(15):7934-7939, April 2013.

[91] B. Kiran, A. K. Kandalam, R. Rallabandi, P. Koirala, X. Li, X. Tang, Y. Wang, H. Fairbrother, G. Gantefoer, and K. Bowen. $(\mathrm{PbS})_{32}$ : A baby crystal. The Journal of Chemical Physics, 136(2):024317, 2012.

[92] J. P. Perdew, J. A. Chevary, S. H. Vosko, K. A. Jackson, M. R. Pederson, D. J. Singh, and C. Fiolhais. Atoms, molecules, solids, and surfaces: Applications of the generalized gradient approximation for exchange and correlation. Physical Review B, 46(11):6671-6687, September 1992.

[93] G.-H. Lee, Y.-J. Yu, C. Lee, C. Dean, K. L. Shepard, P. Kim, and J. Hone. Electron tunneling through atomically flat and ultrathin hexagonal boron nitride. Applied Physics Letters, 99(24):243114, 2011.

[94] L. Britnell, R. V. Gorbachev, R. Jalil, B. D. Belle, F. Schedin, M. I. Katsnelson, L. Eaves, S. V. Morozov, A. S. Mayorov, N. M. R. Peres, A. H. C. Neto, J. Leist, A. K. Geim, L. A. Ponomarenko, and K. S. Novoselov. Electron tunneling through ultrathin boron nitride crystalline barriers. Nano letters, 12(3):17071710, 2012. 
[95] X. Zhong, R. G. Amorim, R. H. Scheicher, R. Pandey, and S. P. Karna. Electronic structure and quantum transport properties of trilayers formed from graphene and boron nitride. Nanoscale, 4(17):5490-5498, 2012.

[96] R. G. Amorim, X. Zhong, S. Mukhopadhyay, R. Pandey, A. R. Rocha, and S. P. Karna. Strain- and electric field-induced band gap modulation in nitride nanomembranes. Journal of Physics: Condensed Matter, 25(19):195801, April 2013.

[97] M. Amman, R. Wilkins, E. Ben-Jacob, P. D. Maker, and R. C. Jaklevic. Analytic solution for the current-voltage characteristic of two mesoscopic tunnel junctions coupled in series. Physical Review B, 43(1):1146, 1991.

[98] J. M. Soler, E. Artacho, J. D. Gale, A. García, J. Junquera, P. Ordejón, and D. Sánchez-Portal. The SIESTA Method for Ab Initio Order-N Materials Simulation. Journal of Physics: Condensed Matter, 14:2745, 2002.

[99] P. Ordejó, D. A. Drabold, M. P. Grumbach, and R. M. Martin. Unconstrained Minimization Approach for Electronic Computations That Scales Linearly with System Size. Physical Review B, 48:14646, 1993. 


\section{Appendix A}

\section{Permission to Republish}

\section{Royal Society of Chemistry}

This appendix documents permission to republish figures 5.12, 5.13.

From: http://pubs.rsc.org/EN/content/requestpermission?msid=c4nr00159a

Reproduced material should be attributed as follows:

For reproduction of material from NJC: Reproduced from Ref. XX with permission from the Centre National de la Recherche Scientifique (CNRS) and The Royal Society of Chemistry.

For reproduction of material from PCCP: Reproduced from Ref. XX with permission from the PCCP Owner Societies.

For reproduction of material from PPS: Reproduced from Ref. XX with permission 
from the European Society for Photobiology, the European Photochemistry Association, and The Royal Society of Chemistry.

For reproduction of material from all other RSC journals and books: Reproduced from Ref. XX with permission from The Royal Society of Chemistry.

If the material has been adapted instead of reproduced from the original RSC publication "Reproduced from" can be substituted with "Adapted from".

In all cases the Ref. XX is the XXth reference in the list of references.

If you are the author of this article you do not need to formally request permission to reproduce figures, diagrams etc. contained in this article in third party publications or in a thesis or dissertation provided that the correct acknowledgement is given with the reproduced material.

Reproduced material should be attributed as follows:

For reproduction of material from NJC: [Original citation] - Reproduced by permission of The Royal Society of Chemistry (RSC) on behalf of the Centre National de la Recherche Scientifique (CNRS) and the RSC

For reproduction of material from PCCP: [Original citation] - Reproduced by permission of the PCCP Owner Societies

For reproduction of material from PPS: [Original citation] - Reproduced by permission of The Royal Society of Chemistry (RSC) on behalf of the European Society for Photobiology, the European Photochemistry Association, and RSC 
For reproduction of material from all other RSC journals: [Original citation] - Reproduced by permission of The Royal Society of Chemistry

If you are the author of this article you still need to obtain permission to reproduce the whole article in a third party publication with the exception of reproduction of the whole article in a thesis or dissertation. 


\section{Appendix B}

\section{Permission to Republish \\ Cambridge University Press}

This appendix documents permission to republish chapter 2 .

From: http://www.cambridge.org/about-us/rights-permissions/ permissions/permissions-requests-our-authors/

In certain circumstances, permissions requests are not required from authors who wish to re-use original material they have written for a Cambridge publication, provided that the subsequent use includes a full acknowledgement of the original publication, together with the copyright notice and the phrase 'Reprinted with permission'.

Permissions requests are waived if:

$\dagger$ The author of the work wishes to reproduce a single chapter (not exceeding 20 per cent of his/her work), journal article or shorter extract in a subsequent 
work (i.e. with a later publication date) of which he or she is to be the author, co-author or editor.

$\dagger$ The author wishes to photocopy a single chapter (not exceeding 20 per cent of his/her work as a whole), journal article or shorter extract for his/her own teaching.

$\dagger$ The author wishes to reproduce a single chapter (not exceeding 20 per cent of his/her published work as a whole), journal article or shorter extract on his/her personal or institution web site for teaching and research purposes.

For all other uses, permission is required, and the author or the author's publisher should refer to the Permissions requests page. 


\section{Appendix C}

\section{Permission to Republish Figures from Lee et al.}

This appendix documents permission to republish figures 2.1 and 2.2. 
License Number

License date

Licensed content publisher

Licensed content publication

Licensed content title

Licensed copyright line

Licensed content author

Licensed content date

Start page

End page

Type of use

Requestor type

Format

Portion

Number of figures/tables

Original Wiley figure/table number(s)

Will you be translating?

Title of your thesis / dissertation

Expected completion date

Expected size (number of pages)

Total
3511150580858

Nov 17, 2014

John Wiley and Sons

Advanced Materials

Room-Temperature Tunneling Behavior of Boron Nitride Nanotubes Functionalized with Gold Quantum Dots

Copyright @ 2013 WILEY-VCH Verlag GmbH

\& Co. KGaA, Weinheim

Chee Huei Lee,Shengyong Qin,Madhusudan A. Savaikar,Jiesheng Wang,Boyi Hao,Dongyan Zhang,Douglas Banyai,John A. Jaszczak,Kendal W. Clark,Juan-Carlos Idrobo,An-Ping Li,Yoke Khin Yap

Jun 17, 2013

4544

4548

Dissertation/Thesis

Author of this Wiley article

Print and electronic

Figure/table

2

Figure 1 Figure 2

No

MULTISCALE MODELING OF ELECTRON TRANSPORT IN NANOSCALE MATERIALS

Dec 2014

150

0.00 USD 


\section{Appendix D}

\section{Permission to Republish Figures from Savaikar et al.}

This appendix documents permission to republish figures 2.3, 2.4, and 2.8. 
License Number

Order Date

Publisher

Publication

Article Title

Author

Online Publication Date

Volume number

Issue number

Type of Use

Requestor type

Format

Portion

Number of figures/tables

Title of your thesis / dissertation

Expected completion date

Estimated size (number of pages)

Total
3511160267024

Nov 17, 2014

AIP Publishing LLC

Journal of Applied Physics

Simulation of charge transport in multi-island tunneling devices: Application to disordered one-dimensional systems at low and high biases Madhusudan A. Savaikar,Douglas Banyai,Paul L. Bergstrom, et al.

Sep 17, 2013

114

11

Thesis/Dissertation

Author (original article)

Print and electronic

Figure/Table

3

MULTISCALE MODELING OF ELECTRON TRANSPORT IN NANOSCALE MATERIALS

Dec 2014

150

0.00 USD 